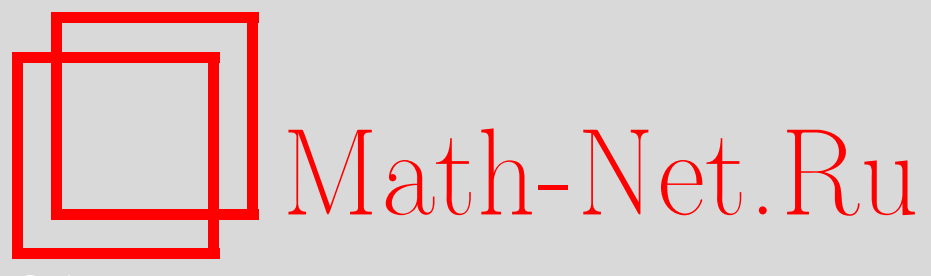

А. Д. Бендиков, А. А. Григорьян, К. Питтэ, В. Вёсс, Изотропные марковские полугруппы на ультраметрических пространствах, УМH, 2014, том 69, выпуск 4, 3-102

DOI: https://doi.org/10.4213/rm9602

Использование Общероссийского математического портала Math-Net.Ru подразумевает, что вы прочитали и согласны с пользовательским соглашением http://www . mathnet.ru/rus/agreement

Параметры загрузки:

IP: 54.147 .182 .235

26 апреля 2023 г., 17:45:00

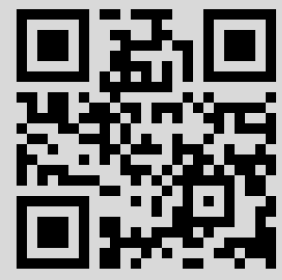




\title{
Изотропные марковские полугруппы на ультраметрических пространствах
}

\author{
А. Д. Бендиков, А. А. Григорьян, К. Питтэ, В. Вёсс
}

Пусть $(X, d)$ - сепарабельное ультраметрическое пространство с компактными шарами. Для заданных эталонной меры $\mu$ на $X$ и функции распределения расстояний $\sigma$ на $[0, \infty)$ строится симметричная марковская полугруппа $\left\{P^{t}\right\}_{t \geqslant 0}$, действующая в $L^{2}(X, \mu)$. Пусть $\left\{\mathscr{X}_{t}\right\}-$ соответствующий марковский процесс. Получены верхние и нижние оценки его переходной плотности и функции Грина, дан критерий его невозвратности, оценены его моменты и описаны марковский генератор $\mathscr{L}$ и его спектр, который является чисто точечным. В частном случае, когда $X=\mathbb{Q}_{p}^{n}$, где $\mathbb{Q}_{p}$ - поле $p$-адических чисел, наша конструкция воспроизводит лапласиан Тайблесона (спектральный множитель) и наша теория также применима к изучению лапласиана Владимирова. Даже в этой хорошо изученной области несколько наших результатов являются новыми. Также изучается связь между марковским процессом $\left\{\mathscr{X}_{t}\right\}$ и процессом Кигами на границе дерева, который индуцирован случайным блужданием на дереве. В заключение приводятся примеры, иллюстрирующие взаимосвязь между операторами дробного дифференцирования и случайными блужданиями.

Библиография: 66 названий.

Ключевые слова: ультраметрическое пространство с мерой, метрические деревья, изотропные марковские полугруппы, марковские генераторы, ядро теплопроводности, переходная плотность, поле $p$-адических чисел, оператор Владимирова-Тайблесона, простое случайное блуждание на дереве, форма Дирихле, гармонические функции конечной энергии, следы гармонических функций конечной энергии.

DOI: $10.4213 / \mathrm{rm} 9602$

Работа была начата и закончена в Университете Билефельда при поддержке Специального исследовательского отдела (SFB 701) Немецкого исследовательского совета. Первый автор поддержан Научно-исследовательским фондом Польского Правительства (грант № 2012/05/B/ST 1/00613). Второй автор поддержан Немецким исследовательским советом (SFB 701). Третий автор поддержан Национальным советом научных исследований (CNRS), Франция. Четвертый автор поддержан Австрийским научным фондом (проекты FWF W1230-N13 и FWF P24028-N18). 


\section{СоДЕРЖАНИЕ}

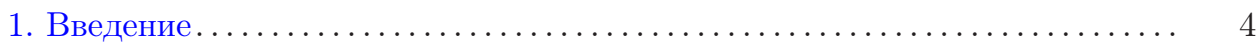

2. Изотропная полугруппа и ядро теплопроводности ............... 11

2.1. Усредняющий оператор ............................ 11

2.2. Основные свойства изотропных полугрупп ............... 12

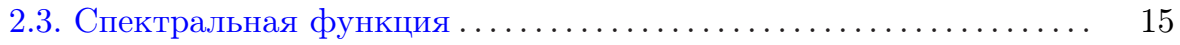

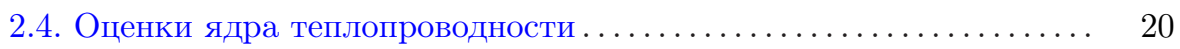

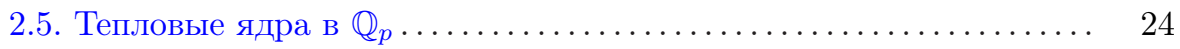

2.6. Функция Грина и невозвратность..................... 28

3. Изотропный лапласиан и его спектр ....................... 31

3.1. Субординация ................................... 31

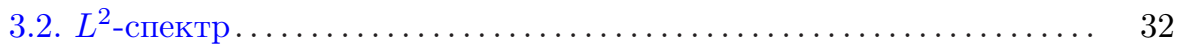

3.3. Форма Дирихле и плотность скачков .................. 38

3.4. $L^{p}$-спектр лапласиана ............................. 40

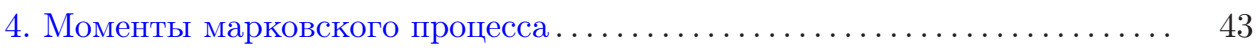

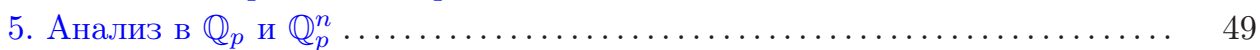

5.1. Оператор $p$-адической дробной производной............... 49

5.2. Сферически симметричные марковские полугруппы ......... 53

5.3. Пространства произведений.................... 57

6. Случайные блуждания на дереве и процессы со скачками на его границе 66

6.1. Корневые деревья и их границы ...................... 66

6.2. Изотропные процессы со скачками на границе дерева......... 70

6.3. Простые случайные блуждания на дереве ................ 70

6.4. Гармонические функции конечной энергии и их граничные зна-

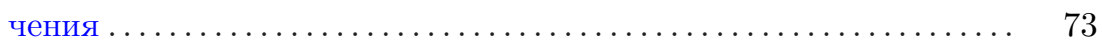

6.5. Процессы со скачками на границе дерева................ 73

7. Двойственность случайных блужданий на деревьях и изотропных про-

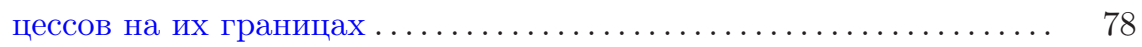

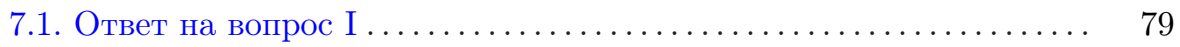

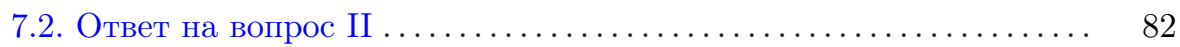

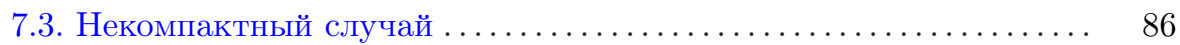

8. Случайное блуждание, связанное с $p$-адической дробной производной . 93

8.1. Оператор $p$-адической дробной производной на $\mathbb{Z}_{p} \ldots \ldots \ldots \ldots . . . . . .93$

8.2. Простое случайное блуждание на корневом дереве $\mathbb{T}_{p}^{o} \ldots \ldots \ldots \ldots 95$

8.3. Случайное блуждание, соответствующее $\mathfrak{D}^{\alpha}$ на $\mathbb{Q}_{p} \ldots \ldots \ldots \ldots .97$

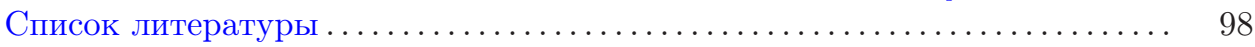

\section{1. Введение}

В последние три десятилетия наблюдается возрастающий интерес к различным конструкциям марковских цепей на ультраметрических (вполне несвязных) пространствах, таких как канторово множество или поле $p$-адических чисел. В этой работе мы вводим и изучаем класс симметричных марковских полугрупп и их марковских генераторов на ультраметрических пространствах. 
Наша конструкция очень прозрачна и приводит к ряду новых результатов, а также к лучшему пониманию ранее известных результатов.

Пусть $(X, d)$ будет метрическим пространством. Метрика $d$ называется ультраметрикой, если она удовлетворяет ультраметрическому неравенству

$$
d(x, y) \leqslant \max \{d(x, z), d(z, y)\}
$$

которое, очевидно, сильнее обычного неравенства треугольника. В этом случае $(X, d)$ называется ультраметрическим пространством.

Мы будем всегда дополнительно предполагать, что рассматриваемое ультраметрическое пространство $(X, d)$ сепарабельно и что каждый замкнутый шар

$$
B_{r}(x)=\{y \in X: d(x, y) \leqslant r\}
$$

компактен. Из последнего условия вытекает, что $(X, d)$ полно.

Из свойства ультраметричности (1.1) следует, что шары в ультраметрическом пространстве $(X, d)$ выглядят совсем не так, как известные всем евклидовы шары. В частности, любые два ультраметрических шара одного радиуса либо не пересекаются, либо совпадают. Следовательно, множество всех различных шаров одного и того же радиуса $r$ образует разбиение пространства $X$.

Одним из наиболее известных примеров ультраметрического пространства является поле $\mathbb{Q}_{p} \quad p$-адических чисел, наделенное $p$-адической нормой $\|x\|_{p}$ и $p$-адической ультраметрикой $d(x, y)=\|x-y\|_{p}$. Более того, для любого целого $n \geqslant 1 \quad p$-адическое $n$-пространство $\mathbb{Q}_{p}^{n}=\mathbb{Q}_{p} \times \cdots \times \mathbb{Q}_{p}$ также является ультраметрическим пространством с ультраметрикой $d_{n}(x, y)$, определяемой равенством

$$
d_{n}(x, y)=\max \left\{d\left(x_{1}, y_{1}\right), \ldots, d\left(x_{n}, y_{n}\right)\right\} .
$$

Если группа изометрий ультраметрического пространства $(X, d)$ действует транзитивно на $X$, то $(X, d)$ на самом деле является локально компактной абелевой группой, что, в частности, имеет место для $\mathbb{Q}_{p}^{n}$.

В литературе различают следующие два подкласса ультраметрических пространств:

(i) $(X, d)$ дискретно и бесконечно;

(ii) $(X, d)$ совершенно (т. е. $X$ не содержит изолированных точек).

Различные конструкции марковских процессов в случае (ii) с дополнительным условием, что $X$ является локально компактной абелевой группой, развивали Ст. Н. Эванс [22], С. Харан [29], [30], Р. С. Исмагилов [33], А. Н. Кочубей [38], [39], С. Альбеверио и В. Карвовски [1], [2], С. Альбеверио и К. Жао [3], М. Дель Муто и А. Фига-Таламанка [42], [43], Х. Х. Родригес-Вега и В. А. Зунига-Галиндо [50], [66]. Они изучали $X$-значные безгранично делимые случайные величины и процессы, используя средства анализа Фурье; ссылки общего характера см. в книгах Э. Хьюитта и К. А. Росса [31], М. Х. Тайблесона [55] и А.Н. Кочубея [39]. Отметим, что спектральные множители Тайблесона на $\mathbb{Q}_{p}^{n}$ являются ранними предшественниками рассматриваемых здесь лапласианов.

Дж. Пирсон и Дж. Беллиссард [45] и Дж. Кигами [36], [37] рассматривали случайные блуждания на канторовом множестве и соответственно на канторовом множестве с выколотой точкой. В [36], [37] основное внимание уделялось 
взаимосвязи между случайными блужданиями на деревьях и процессами со скачками на их границах. В этом контексте упомянем также работы Д. Алдуса и Ст. Н. Эванса [4] и 3.-К. Чена, М. Фукушимы и Дж. Йинга [15]. Мы вернемся к работе Дж. Кигами в последних трех разделах настоящей работы.

Совершенно иной подход развивался В. С. Владимировым, И. В. Воловичем и Е. И. Зеленовым [57], [59]. Они занимались р-адическим анализом (распределения Брюа, преобразования Фурье и т. п.), связанным с концепцией $p$-адической квантовой механики, и ввели класс псевдодифференциальных операторов на $\mathbb{Q}_{p}$ и на $\mathbb{Q}_{p}^{n}$. В частности, они изучали $p$-адический лапласиан, определяемый на $\mathbb{Q}_{p}^{3}$, и соответствующее $p$-адическое уравнение Шрёдингера. В частности, они в явном виде вычислили (в виде разложения в ряд) некоторые ядра теплопроводности, а также функцию Грина $p$-адического лапласиана. В связи с теорией псевдодифференциальных операторов на произвольных вполне несвязных группах упомянем здесь раннюю работу Л. Салофф-Коста [51].

Дискретные ультраметрические пространства $(X, d)$ (как в (i)) рассматривались в работе А. Бендикова, А. Григорьяна и К. Питтэ [7], непосредственно предшествующей настоящей работе. Среди примеров таких пространств упомянем класс локально конечных групп: счетная группа $G$ локально конечна, если любое ее конечное подмножество порождает конечную подгруппу. Каждая локально конечная группа $G$ является объединением возрастающей последовательности конечных подгрупп $\left\{G_{n}\right\}$. Ультраметрику $d$ в $G$ можно определить так: $d(x, y)$ - минимальное значение $n$, для которого $x$ и $y$ принадлежат одному и тому же классу смежности группы $G_{n}$.

Поскольку локально конечные группы не являются конечно порожденными, основные понятия геометрической теории групп, такие как словарная метрика, рост объема, изопериметрические неравенства и т. п. (ср., например, с [16], [28], [46]-[48], [52], [56], [61]), в этой постановке не применимы. Вместо словарной метрики в этой ситуации может быть использовано понятие ультраметрики (см. [5]-[7]).

Выбирая генерирующее множество для каждой подгруппы $G_{n}$ локально конечной группы $G$, мы тем самым определяем случайное блуждание, т. е. марковское ядро на $G_{n}$. Беря выпуклую комбинацию марковских ядер по всем $G_{n}$, получаем марковское ядро на $G$, которое определяет случайное блуждание на $G$. Такие случайные блуждания изучали Д. А. Дарлинг и П. Эрдеш [17], Х. Кестен и Ф. Спицер [35], Л. Флатто и Дж. Питт [26], Н. Фериг и С. А. Молчанов [25], М. А. Касымджанова [34], Д. И. Картрайт [13], Г. Ф. Лоулер [40], С. Брофферио и В. Вёсс [11], см. также работу А. Бендикова и Л. СалоффКоста [9]. В частности, работа [40] содержит замечательный общий критерий возвратности таких случайных блужданий. Дальнейшие результаты о марковских процессах на ультраметрических пространствах можно найти в [18], [19], [23], [24], [41], [49].

Многие из результатов в вышеупомянутых работах покрываются нашим подходом через ультраметрики. Мы развиваем средства для анализа одного естественного класса марковских процессов на ультраметрических пространствах, не предполагая наличия какой-либо групповой структуры. В частности, характер наших рассуждений позволяет внести в рассмотрение произвольную меру 
Радона $\mu$ на $X$ (вместо меры Хаара в случае групп), которая используется как мера скорости для марковского процесса.

Итак, для заданного ультраметрического пространства $(X, d)$ зафиксируем меру Радона $\mu$ на $X$ с полным носителем и определим семейство $\left\{\mathrm{Q}_{r}\right\}_{r>0}$ усредняющих операторов, действующих на неотрицательных или ограниченных борелевских функциях $f: X \rightarrow \mathbb{R}$, полагая

$$
\mathrm{Q}_{r} f(x)=\frac{1}{\mu\left(B_{r}(x)\right)} \int_{B_{r}(x)} f d \mu .
$$

Заметим, что $0<\mu\left(B_{r}(x)\right)<\infty$ для всех $x \in X$ и $r>0$. Оператор $\mathrm{Q}_{r}$ имеет ядро

$$
K_{r}(x, y)=\frac{1}{\mu\left(B_{r}(x)\right)} \mathbf{1}_{B_{r}(x)}(y) .
$$

Оно симметрично относительно $x, y$, поскольку $B_{r}(x)=B_{r}(y)$ для любого $y \in$ $B_{r}(x)$. Ясно, что $\mathrm{Q}_{r}$ является марковским оператором на пространстве $\mathscr{B}_{b}(X)$ ограниченных борелевских функций на $X$, т. е. $\mathrm{Q}_{r} f \geqslant 0$, если $f \geqslant 0, \mathrm{Q}_{r} 1=1$. Отсюда следует, что $Q_{r}$ продолжается до ограниченного самосопряженного оператора в $L^{2}(X, \mu)$.

Выберем функцию $\sigma$, удовлетворяющую следующим предположениям:

$\sigma:[0, \infty] \rightarrow[0,1]$ - строго монотонно возрастающая непрерывная слева

$$
\text { функция, для которой } \sigma(0+)=0 u \sigma(\infty)=1 \text {. }
$$

Тогда оператор

$$
P f=\int_{0}^{\infty} \mathrm{Q}_{r} f d \sigma(r)
$$

как и $\mathrm{Q}_{r}$, является марковским оператором в $\mathscr{B}_{b}(X)$, а также ограниченным самосопряженным оператором в $L^{2}(X, \mu)$.

Оператор $P$ определяет марковскую цепь с дискретным временем $\left\{\mathscr{X}_{n}\right\}_{n \in \mathbb{N}}$ на $X$ со следующим правилом перехода: случайная точка $\mathscr{X}_{n+1}$ является $\mu$-равномерно распределенной в $B_{r}\left(\mathscr{X}_{n}\right)$, где радиус $r$ выбирается случайным образом в соответствии с вероятностным распределением $\sigma$. По этой причине мы называем $\sigma$ функиией распределения расстояний.

Заметим, что оператор $P$ определяется тройкой $(d, \mu, \sigma)$. Мы называем $P$ изотропным марковским оператором, связанным с $(d, \mu, \sigma)$. Изотропный марковский оператор $P$ обладает уникальными свойствами, проистекающими из ультраметрического свойства. Прежде всего отметим простое тождество

$$
\mathrm{Q}_{r} \mathrm{Q}_{s}=\mathrm{Q}_{s} \mathrm{Q}_{r}=\mathrm{Q}_{\max \{r, s\}} .
$$

Действительно, в любом шаре $B$ радиуса $r$ любая точка $x \in B$ является центром $B$. Поскольку значение $\mathrm{Q}_{r} f(x)$ есть среднее значение функции $f$ в $B$, мы видим, что $\mathrm{Q}_{r} f(x)$ не зависит от $x \in B$; т. е. $\mathrm{Q}_{r} f=$ const на $B$. Если $s \leqslant r$, то применение $\mathrm{Q}_{s}$ к $\mathrm{Q}_{r} f$ не изменяет эту константу, откуда получаем $\mathrm{Q}_{s} \mathrm{Q}_{r} f=\mathrm{Q}_{r} f$. С другой стороны, если $s>r$, то любой шар радиуса $s$ является объединением конечного числа непересекающихся шаров радиуса $r$. 
Так как интегралы от $f$ и $\mathrm{Q}_{r} f$ по любому такому шару равны, мы получим $\mathrm{Q}_{s} \mathrm{Q}_{r} f=\mathrm{Q}_{s} f$.

Поскольку в силу (1.7) $\mathrm{Q}_{r}^{2}=\mathrm{Q}_{r}$, мы получаем, что $\mathrm{Q}_{r}$ является ортопроектором ${ }^{1}$ в $L^{2}$. В частности, spec $\mathrm{Q}_{r} \subset[0,1]$.

Из (1.6) следует, что спектральные проекторы в спектральном разложении оператора $P$ являются усредняющими операторами $Q_{r}$ с точностью до замены переменных (ср. с (2.6)). Тот факт, что спектральные проекторы сами являются марковскими операторами, привносит новое понимание, новые технические возможности и результаты нового типа, которые не имеют аналога в других ситуациях, где речь идет о более привычных марковских процессах.

В частности, марковский оператор $P$ неотрицательно определен, что позволяет определить степени $P^{t}$ для всех $t \geqslant 0$. Тогда $\left\{P^{t}\right\}_{t \geqslant 0}-$ симметричная сильно непрерывная марковская полугруппа. Из (1.6) следует, что $P^{t}$ допускает при $t>0$ следующее представление:

$$
P^{t} f(x)=\int_{0}^{\infty} \mathrm{Q}_{r} f(x) d \sigma^{t}(r)
$$

С другой стороны, можно определить $P^{t}$ формулой (1.8), а затем использовать формулу (1.7) для доказательства того, что $P^{s} P^{t}=P^{s+t}$.

Полугруппа $\left\{P^{t}\right\}_{t \geqslant 0}$ определяет марковский процесс с непрерывным временем $\left\{\mathscr{X}_{t}\right\}_{t \geqslant 0}$. Поскольку $n$-й переходный оператор марковской цепи с дискретным временем $\left\{\mathscr{X}_{n}\right\}_{n \in \mathbb{N}}$ есть $P^{n}$, видим, что марковская цепь с дискретным временем совпадает с ограничением марковского процесса с непрерывным временем $\left\{\mathscr{X}_{t}\right\}$ на целые значения $t$. Это позволяет сосредоточиться только на изучении процесса с непрерывным временем $\left\{\mathscr{X}_{t}\right\}_{t \geqslant 0}$.

Мы называем марковскую полугруппу $\left\{P^{t}\right\}_{t \geqslant 0}$, определяемую формулами (1.3)-(1.8), изотропной полугруппой, а процесс со скачками $\left\{\mathscr{X}_{t}\right\}_{t \geqslant 0}$ uзотропным прочессом, связанным с тройкой $(d, \mu, \sigma)$.

Кратко опишем содержание настоящей работы, которая посвящена изучению изотропных полугрупп.

В разделе 2 строится вышеупомянутая изотропная полугруппа и приводятся явные формулы для ее ядра теплопроводности $p(t, x, y)$ (т. е. переходной плотности процесса $\left\{\mathscr{X}_{t}\right\}$ ). Как указано выше, наш подход основан на наблюдении, что составляющие блоки оператора $P$, а именно усредняющие операторы $\mathrm{Q}_{r}$ из (1.3), являются ортогональными проекторами в $L^{2}(X, \mu)$, что позволяет задействовать на ранней стадии методы спектральной теории и функционального исчисления.

Устанавливаются некоторые основные свойства ядра теплопроводности, например его непрерывность вне диагонали, и доказываются верхние и нижние оценки в терминах $t$ и $d(x, y)$.

$\mathrm{K}$ примеру, в $\mathbb{Q}_{p}$ с $p$-адической ультраметрикой $\|x-y\|_{p}$ и мерой Хаара $\mu$ естественным выбором функции распределения расстояний является

$$
\sigma(r)=\exp \left(-\left(\frac{p}{r}\right)^{\alpha}\right), \quad \alpha>0 .
$$

\footnotetext{
${ }^{1}$ Для сравнения упомянем, что аналогичный усредняющий оператор в $\mathbb{R}^{n}$ также ограничен и самосопряжен, но имеет непустую отрицательную часть спектра. В частности, он не является ортопроектором.
} 
В этом случае соответствующее ядро теплопроводности допускает оценку

$$
p_{t}(x, y) \simeq \frac{t}{\left(t^{1 / \alpha}+\|x-y\|_{p}\right)^{1+\alpha}}
$$

для всех $t>0$ и $x, y \in \mathbb{Q}_{p}$. Заметим, что оценка (1.10) напоминает оценку ядра теплопроводности для симметричного $\alpha$-устойчивого процесса в $\mathbb{R}$.

Также получены явное выражение для функции Грина изотропной полугруппы и критерий невозвратности в терминах роста объема. В отличие от ранее известных критериев невозвратности (ср. с [40]) наш критерий не предполагает никакой групповой структуры.

В разделе 3 рассматриваются спектральные свойства изотропного лапласиана $\mathscr{L}$, который по определению является (положительно определенным) генератором изотропной полугруппы, т. е. $P^{t}=e^{-t \mathscr{L}}$. Дается полное описание спектра $\mathscr{L}$, в частности, показывается, что спектр является чисто точечным. Приводится также явная форма всех собственных функций оператора $\mathscr{L}$ и показывается, что спектры его продолжений в пространствах $L^{p}, 1 \leqslant p<\infty$, не зависят от $p$.

Необычным свойством изотропного лапласиана $\mathscr{L}$ является то, что для любой возрастающей биекции $\psi:[0, \infty) \rightarrow[0, \infty)$ оператор $\psi(\mathscr{L})$ также является изотропным лапласианом (для другой функции распределения расстояний). В частности, $\mathscr{L}^{\alpha}$ - изотропный лапласиан для любого $\alpha>0$. Напомним для сравнения, что для произвольного симметричного марковского генератора $\mathscr{L}$ оператор $\mathscr{L}^{\alpha}$ порождает марковскую полугруппу только при $0<\alpha \leqslant 1$.

В разделе 4 получены двусторонние оценки моментов изотропного процесca $\left\{\mathscr{X}_{t}\right\}$.

В случае, когда $X$ - локально компактная группа, наши результаты применимы с произвольной мерой Радона $\mu$ вместо меры Хаара. Некоторые из вышеупомянутых вопросов, например оценки ядра теплопроводности и функции Грина, особенно чувствительны к выбору меры $\mu$. С другой стороны, спектр лапласиана и оценки моментов не зависят от $\mu$. Эти величины сильно зависят от выбора ультраметрики $d$, в то время как собственные функции зависят и от $d$, и от $\mu$.

В разделе 5 мы сравниваем наш изотропный лапласиан с другими ранее известными “дифференциальными" операторами в $\mathbb{Q}_{p}$ и $\mathbb{Q}_{p}^{n}$. Понятие оператора дробной производной $\mathfrak{D}^{\alpha}$ на функциях, определенных на $\mathbb{Q}_{p}$, было введено В. С. Владимировым [57] с помощью преобразования Фурье в $\mathbb{Q}_{p}$. Оператор $\mathfrak{D}^{\alpha}$ совпадает с оператором Тайблесона [55], введенным в совершенно ином контексте как множитель Рисса на $\mathbb{Q}_{p}^{n}$. Мы показываем, что $\mathfrak{D}^{\alpha}$ совпадает с нашим изотропным лапласианом $\mathscr{L}_{\alpha}$, связанным с функцией распределения расстояний (1.9). В частности, отсюда следует, что ядро теплопроводности оператора $\mathfrak{D}^{\alpha}$ удовлетворяет оценке (1.10). Заметим, что ранее была известна только верхняя оценка для ядра теплопроводности оператора $\mathfrak{D}^{\alpha}$ (ср. с [39; гл. 4.1, лемма 4.1]). Мы также даем простое доказательство ранее известной явной формулы для функции Грина оператора $\mathfrak{D}^{\alpha}$.

Используя функциональное исчисление оператора $\mathfrak{D}^{1}$, мы даем полное описание класса всех инвариантных относительно вращений марковских генерато- 
ров на $\mathbb{Q}_{p}$. Этот класс включает изотропные лапласианы, но не исчерпывается ими. Как следствие, мы получаем, что класс всех инвариантных относительно вращений марковских процессов в $\mathbb{Q}_{p}$ совпадает с классом марковских процессов, построенным С. Альбеверио и В. Карвовски [2] путем использования гораздо более сложных технических средств.

Далее мы рассматриваем операторы “частных производных" на $\mathbb{Q}_{p}^{n} \cdot p$-адический лапласиан Владимирова на $\mathbb{Q}_{p}^{n}$ определяется как прямая сумма операторов $\mathfrak{D}^{\alpha}$, действующих раздельно по каждой координате. Хотя этот оператор не является изотропным лапласианом, его можно изучать в рамках нашей теории, что дает простые прямые доказательства многих результатов из [59], не использующие анализа Фурье и теории распределений Брюа.

Еще одно многомерное обобщение оператора $\mathfrak{D}^{\alpha}-$ это оператор Тайблесона $\mathfrak{T}^{\alpha}$ в $\mathbb{Q}_{p}^{n}$, который определяется с помощью преобразования Фурье в $\mathbb{Q}_{p}^{n}$. Мы показываем, что оператор $\mathfrak{T}^{\alpha}$ является изотропным лапласианом, что позволяет получить точные аналитические результаты.

В разделе 6 мы используем тот факт, что каждое локально компактное ультраметрическое пространство возникает как граница локально конечного дерева. С помощью этого факта мы связываем случайные блуждания ${ }^{2}$ на дереве с изотропными процессами на его границе. Дж. Кигами в [36] начинает с невозвратного простого случайного блуждания на дереве и строит естественно связанный с ним процесс со скачками на границе дерева: имея форму Дирихле случайного блуждания на дереве, процесс на границе индуцирован формой Дирихле, которая воспроизводит энергию гармонической функции на дереве через ее граничные значения. Этот подход аналогичен известному интегралу Дугласа [21] в единичном диске. Используя его, Дж. Кигами [36] предпринимает подробный анализ процесса на границе.

Рассматривая сначала компактный случай, мы отвечаем в разделе 7 на очевидный вопрос о том, как связан подход Дж. Кигами с нашим подходом. Связь в основном взаимно однозначна: каждый граничный процесс, индуцированный случайным блужданием, является изотропным процессом в нашем понимании. Обратно, мы показываем, что с точностью до единственной линейной замены времени каждый изотропный процесс на границе дерева возникает из однозначно определенного случайного блуждания как в [36]. Дополнительно мы объясняем, как граничный процесс на дереве преобразуется в изотропный процесс на некомпактном ультраметрическом пространстве, заданном проколотой границей дерева. Это следует сравнить с [37].

Наконец, в разделе 8 мы явно строим случайные блуждания на деревьях, которые соответствуют операторам $p$-адической дробной производной на (компактной) группе $\mathbb{Z}_{p} \quad p$-адических целых чисел и на всем $\mathbb{Q}_{p}$.

Авторы благодарят С. Альбеверио, Дж. Беллиссарда, П. Диакониса, В. Херфорта, А. Н. Кочубея, С. А. Молчанова, Л. Салофф-Коста, И. В. Воловича и Е. И. Зеленова за плодотворные обсуждения и ценные замечания.

\footnotetext{
${ }^{2}$ Простые случайные блуждания с дискретным временем на дереве очень хорошо изучены - см. главу 9 в книге В. Вёсса [63].
} 


\section{2. Изотропная полугруппа и ядро теплопроводности}

Повсюду в этой работе $(X, d)$ - ультраметрическое пространство, которое предполагается сепарабельным и таким, что все замкнутые $d$-шары $B_{r}(x)$ компактны.

2.1. Усредняющий оператор. Напомним, что для любого $r>0$ оператор

$$
\mathrm{Q}_{r} f(x)=\frac{1}{\mu\left(B_{r}(x)\right)} \int_{B_{r}(x)} f d \mu
$$

является ортопроектором в $L^{2} \equiv L^{2}(X, \mu)$ (ср. с $\left.(1.3)\right)$ и образ оператора $\mathrm{Q}_{r}$ является подпространством $\mathscr{V}_{r}$ пространства $L^{2}$, состоящим из всех функций, принимающих постоянные значения на каждом шаре радиуса $r$.

Ясно, что семейство $\left\{\mathscr{V}_{r}\right\}_{r>0}$ монотонно убывает относительно включений множеств. Следовательно, существует предел

$$
\mathrm{Q}_{\infty}:=s-\lim _{r \rightarrow \infty} \mathrm{Q}_{r}
$$

в сильной операторной топологии, который является ортопроектором на $\mathscr{V}_{\infty}=$ $\bigcap_{r>0} \mathscr{V}_{r}$. Значит, $\mathscr{V}_{\infty}$ состоит из постоянных функций. Если $\mu(X)=\infty$, то $\mathscr{V}_{\infty}=\{0\}$ и $\mathrm{Q}_{\infty}=0$, в то время как в случае $\mu(X)<\infty$ имеем $\operatorname{dim} \mathscr{V}_{\infty}=1$ и

$$
\mathrm{Q}_{\infty} f=\frac{1}{\mu(X)} \int_{X} f d \mu .
$$

Положим также $\mathrm{Q}_{0}:=\mathrm{id}$.

Лемма 2.1. Семейство $\left\{\mathrm{Q}_{r}\right\}_{r \in[0, \infty)}$ ортопроекторов сильно непрерывно справа nо $r$.

ДокАзАТЕльство. Сначала покажем, что $r \mapsto \mathrm{Q}_{r}$ сильно непрерывно в $r=0$, т. e.

$$
s-\lim _{s \rightarrow 0+} \mathrm{Q}_{s}=\mathrm{id} .
$$

Пусть $f$ - непрерывная функция на $X$ с компактным носителем. Тогда для любого $x \in X$ имеем

$$
\mathrm{Q}_{s} f(x) \rightarrow f(x) \quad \text { при } s \rightarrow 0 .
$$

Поскольку семейство $\left\{\mathrm{Q}_{s} f\right\}_{s \in(0,1)}$ равномерно ограничено величиной $\sup |f|$ и имеет равномерно компактные носители, по теореме о мажорируемой сходимости следует, что

$$
\left\|\mathrm{Q}_{s} f-f\right\|_{L^{2}} \rightarrow 0 \quad \text { при } s \rightarrow 0 .
$$

Поскольку пространство непрерывных функций с компактным носителем всюду плотно в $L^{2}$, соотношение (2.3) распространяется на все $f \in L^{2}$, откуда следует (2.2).

Далее, докажем, что $r \mapsto \mathrm{Q}_{r}$ сильно непрерывно справа при любом $r>0$, т. e.

$$
s-\lim _{s \rightarrow r+} \mathrm{Q}_{s}=\mathrm{Q}_{r} .
$$


Достаточно показать, что

$$
\left\|\mathrm{Q}_{s} f-\mathrm{Q}_{r} f\right\|_{L^{2}} \rightarrow 0 \quad \text { при } s \rightarrow r+
$$

для любой непрерывной функции $f$ с компактным носителем. Действительно, для любого $x \in X$ функция $r \mapsto \mathrm{Q}_{r} f(x)$ непрерывна справа в силу (1.3) (так как шары замкнуты), откуда (2.5) следует по теореме о мажорируемой сходимости. Лемма доказана.

Для любого $\lambda \in \mathbb{R}$ положим

$$
E_{\lambda}= \begin{cases}\mathrm{Q}_{1 / \lambda}, & \lambda>0, \\ 0, & \lambda \leqslant 0 .\end{cases}
$$

Заметим, что $E_{0+}=\mathrm{Q}_{\infty}$. Из вышеупомянутых свойств оператора $\mathrm{Q}_{r}$ следует, что семейство $\left\{E_{\lambda}\right\}$ ортопроекторов в $L^{2}$ является непрерывным слева разложением единицы. Следовательно, для любой борелевской функции $\varphi:[0, \infty) \rightarrow \mathbb{R}$ интеграл

$$
\int_{[0, \infty)} \varphi(\lambda) d E_{\lambda}
$$

определяет самосопряженный неотрицательно определенный оператор, который ограничен тогда и только тогда, когда $\varphi$ ограничена.

2.2. Основные свойства изотропных полугрупп. Теперь рассмотрим оператор $P$, определенный равенством (1.6) с функцией $\sigma$, удовлетворяющей условиям (1.5). Заметим, что интеграл в (1.6) сходится в сильной операторной топологии, так как

$$
\int_{0}^{\infty}\left\|\mathrm{Q}_{r} f\right\|_{L^{2}} d \sigma(r)<\infty
$$

для любой функции $f \in L^{2}$. С другой стороны, для любой $f \in \mathscr{B}_{b}(X)$ интеграл (1.6) сходится поточечно. Более того, в этом случае функция $P f$ непрерывна, потому что функция

$$
x \mapsto \int_{\varepsilon}^{\infty} \mathrm{Q}_{r} f(x) d \sigma(r)
$$

для любого $\varepsilon>0$ локально постоянна и, значит, она непрерывна и равномерно сходится к $P f(x)$ при $\varepsilon \rightarrow 0$.

Как уже было отмечено, $P$ - самосопряженный оператор в $L^{2}$ и $\operatorname{spec} P \subset$ $[0,1]$. В частности, для любого $t>0$ корректно определена степень $P^{t}$. Положим также $P^{0}:=\mathrm{id}$. В следующем утверждении собраны основные свойства оператора $P^{t}$.

Теорема 2.2. (а) Семейство $\left\{P^{t}\right\}_{t \geqslant 0}$ является сильно непреръвной симметричной марковской полугруппой на $L^{2}(X, \mu)$.

(b) Для любого $t>0$ оператор $P^{t}$ имеет представление (1.8), m.е.

$$
P^{t} f=\int_{[0, \infty)} \mathrm{Q}_{r} f d \sigma^{t}(r) .
$$


(с) Для любого $t>0$ оператор $P^{t}$ допускает интегралъное ядро $p(t, x, y)$, m.е. для всех $f \in \mathscr{B}_{b}$ u $f \in L^{2}$

$$
P^{t} f(x)=\int_{X} p(t, x, y) f(y) d \mu(y)
$$

где $p(t, x, y)$ задается равенством

$$
p(t, x, y)=\int_{[d(x, y), \infty)} \frac{d \sigma^{t}(r)}{\mu\left(B_{r}(x)\right)} .
$$

Функция $p(t, x, y)$ называется ядром теплопроводности полугруппы $\left\{P^{t}\right\}$. Из (2.8) ясно, что $p(t, x, y)<\infty$ для всех $t>0$ и $x \neq y$, в то время как при определенных условиях $p(t, x, x)$ может быть равно $\infty$.

Для $f \in \mathscr{B}_{b}$ тождество (2.7) выполняется поточечно, т. е. для всех $x \in X$, в то время как для $f \in L^{2}$ оно является тождеством двух $L^{2}$-функций, т. е. выполняется для $\mu$-почти всех $x$.

ДокАзАТЕЛьство. Интегрирование по частям в (1.6) дает

$$
P f=\int_{[0, \infty)} \mathrm{Q}_{r} f d \sigma(r)=\mathrm{Q}_{\infty} f-\int_{(0, \infty)} \sigma(r) d \mathrm{Q}_{r} f
$$

для любой $f \in L^{2}$. Делая замену $\lambda=1 / r$ и используя (2.6), получаем

$$
P f=E_{0+} f+\int_{(0, \infty)} \sigma\left(\frac{1}{\lambda}\right) d E_{\lambda} f=\int_{[0, \infty)} \sigma\left(\frac{1}{\lambda}\right) d E_{\lambda} f,
$$

где мы полагаем $\sigma(\infty)=1$. Отсюда получаем спектральное разложение оператора $P$ в следующем виде:

$$
P=\int_{[0, \infty)} \sigma\left(\frac{1}{\lambda}\right) d E_{\lambda}
$$

Следовательно,

$$
P^{t}=\int_{[0, \infty)} \sigma^{t}\left(\frac{1}{\lambda}\right) d E_{\lambda} .
$$

(а) Полугрупповое тождество $P^{t} P^{s}=P^{t+s}$ является непосредственным следствием (2.11) и функционального исчисления. Условие строгой непрерывности

$$
s-\lim _{t \rightarrow 0+} P^{t}=\mathrm{id}
$$

также следует из $(2.11)$, поскольку $\sigma(1 / \lambda)>0$ при $\lambda \in[0, \infty)$ и, тем самым, $\sigma^{t}(1 / \lambda) \rightarrow 1$ при $t \rightarrow 0+$.

(b) Обращая рассуждение в выводе (2.11) из (2.9), получаем, что (2.11) влечет

$$
P^{t} f=\int_{[0, \infty)} \mathrm{Q}_{r} f d \sigma^{t}(r)
$$


(c) Из (b), (1.3) и теоремы Фубини следует, что для любой $f \in \mathscr{B}_{b}$

$$
\begin{aligned}
P^{t} f(x) & =\int_{[0, \infty)}\left(\frac{1}{\mu\left(B_{r}(x)\right)} \int_{X} \mathbf{1}_{B_{r}(x)}(y) f(y) d \mu(y)\right) d \sigma^{t}(r) \\
& =\int_{X}\left(\int_{[d(x, y), \infty)} \frac{1}{\mu\left(B_{r}(x)\right)} d \sigma^{t}(r)\right) f(y) d \mu(y) \\
& =\int_{X} p(t, x, y) f(y) d \mu(y) .
\end{aligned}
$$

Для $f \in L^{2}$ утверждение доказывается посредством стандартной аппроксимации.

Теорема доказана.

ЗАмЕчАниЕ 2.3. В доказательстве теоремы 2.2 мы не использовали в полную силу тот факт, что $\sigma$ является строго монотонно возрастающей функцией (cp. с (1.5)). Для этой теоремы достаточно предполагать, что $\sigma$ монотонно возрастает и $\sigma(r)>0$ при $r>0$.

ЗАмечАниЕ 2.4. Если взять (1.8) в качестве определения оператора $P^{t}$, то полугрупповое тождество $P^{t} P^{s}=P^{t+s}$ можно доказать с помощью (1.7). Действительно, для любых заданных $s, t>0$ и $f \in L^{2}$ имеем

$$
\begin{aligned}
P^{s} P^{t} f(x) & =\int_{0}^{\infty} d \sigma^{s}(r) \int_{0}^{\infty} d \sigma^{t}\left(r^{\prime}\right) \mathrm{Q}_{r} \mathrm{Q}_{r^{\prime}} f(x) \\
& =\int_{0}^{\infty} d \sigma^{s}(r) \int_{0}^{\infty} d \sigma^{t}\left(r^{\prime}\right) \mathrm{Q}_{\max \left\{r, r^{\prime}\right.} f(x) .
\end{aligned}
$$

Пусть $\xi_{1}$ и $\xi_{2}$ - две независимые случайные величины с распределениями $\sigma^{s}$ и $\sigma^{t}$ соответственно. Тогда распределением случайной величины $\xi=\max \left\{\xi_{1}, \xi_{2}\right\}$ является $\sigma^{t+s}$. Следовательно,

$$
P^{s} P^{t} f(x)=\mathrm{E}\left(\mathrm{Q}_{\max \left\{\xi_{1}, \xi_{2}\right\}} f(x)\right)=\int_{0}^{\infty} \mathrm{Q}_{r} f(x) d \sigma^{t+s}(r)=P^{t+s} f(x) .
$$

СлЕДСТВиЕ 2.5. Для всех $x, y \in X u t>0$ имеем $p(t, x, y)>0$,

$$
p(t, x, y)=p(t, y, x)
$$

u

$$
p(t, x, y) \leqslant \min \{p(t, x, x), p(t, y, y)\} .
$$

ДокАЗАТЕЛьство. Положительность $p(t, x, y)$ следует из $(2.8)$ и строгой монотонности $\sigma$.

В интеграле (2.8) имеем $r \geqslant d(x, y)$, откуда следует, что $B_{r}(x)=B_{r}(y)$ и $p(t, x, y)=p(t, y, x)$. Симметрия ядра теплопроводности также следует из того, что $P^{t}$ самосопряжен.

В силу (2.8) имеем

$$
p(t, x, y)=\int_{[d(x, y), \infty)} \frac{d \sigma^{t}(r)}{\mu\left(B_{r}(x)\right)} \leqslant \int_{[0, \infty)} \frac{d \sigma^{t}(r)}{\mu\left(B_{r}(x)\right)}=p(t, x, x),
$$

откуда следует (2.12). Следствие доказано. 
Заметим, что в общем случае ядро теплопроводности удовлетворяет лишь оценке

$$
p(t, x, y) \leqslant \sqrt{p(t, x, x) p(t, y, y)} .
$$

Оценка (2.12), очевидно, сильнее, что отражает особое свойство ультраметрики.

СлЕДСтвиЕ 2.6. Для любого $t>0$ функиия

$$
x, y \mapsto \begin{cases}\frac{1}{p(t, x, y)}, & x \neq y, \\ 0, & x=y,\end{cases}
$$

является ультраметрикой.

ДокАЗАТЕЛЬСтво. Положим

$$
F(x, r)=\left(\int_{[r,+\infty)} \frac{d \sigma^{t}(s)}{\mu\left(B_{s}(x)\right)}\right)^{-1} \text { при } r>0,
$$

$F(x, 0)=0$ и отметим следующие два свойства функции $F$ :

(a) $r \mapsto F(x, r)$ монотонно возрастает по $r$;

(b) $F(x, r)=F(y, r)$ при условии $r \geqslant d(x, y)$, так как в этом случае $B_{s}(x)=$ $B_{s}(y)$ для всех $s \geqslant r$.

Для любой функции $F$ с этими свойствами $\rho(x, y):=F(x, d(x, y))$ является ультраметрикой, так как симметрия следует из (b), а ультраметрическое неравенство (1.1) следует из (а) и $(\mathrm{b}):$ если $d(x, y) \leqslant d(x, z)$, то

$$
\rho(x, y)=F(x, d(x, y)) \leqslant F(x, d(x, z))=\rho(x, z),
$$

а если $d(x, y) \leqslant d(y, z)$, то

$$
\rho(x, y)=F(y, d(x, y)) \leqslant F(y, d(y, z))=\rho(y, z) .
$$

Следствие доказано.

2.3. Спектральная функция. Для марковской полугруппы $P$, связанной с тройкой $(d, \mu, \sigma)$, определим внутреннюю ультраметрику $d_{*}$ равенством

$$
\frac{1}{d_{*}(x, y)}=\log \frac{1}{\sigma(d(x, y))} .
$$

Поскольку $d_{*}$ выражается в виде строго монотонно возрастающей функции от $d$, обращающейся в нуль в нуле, получаем, что $d_{*}$ является ультраметрикой на $X$. Обозначим через $B_{r}^{*}(x)$ метрические шары, определенные ультраметрикой $d_{*}$.

Лемма 2.7. Для любого $r \geqslant 0$ положим

$$
s=\frac{1}{\log (1 / \sigma(r))} .
$$

Тогда следующее тождество выполняется для всех $x \in X$ :

$$
B_{s}^{*}(x)=B_{r}(x) .
$$

Следовательно, метрики $d u d_{*}$ определяют одно и то же множество шаров и одну и ту же топологию. 
ДокАЗАТЕЛЬСтво. Имеем

$$
\begin{aligned}
B_{s}^{*}(x) & =\left\{y \in X: d_{*}(x, y) \leqslant s\right\} \\
& =\{y \in X: \sigma(d(x, y)) \leqslant \sigma(r)\} \\
& =\{y \in X: d(x, y) \leqslant r\} \\
& =B_{r}(x),
\end{aligned}
$$

где мы использовали тот факт, что $\sigma$ строго монотонно возрастает. Лемма доказана.

ОПРЕДЕЛЕНИЕ 2.8. Для любого $x \in X$ определим спектралъную функиию $N(x, \cdot):[0, \infty) \rightarrow[0, \infty)$ формулой

$$
N(x, \tau)=\frac{1}{\mu\left(B_{1 / \tau}^{*}(x)\right)}
$$

(см. рис. 1, 2 и 3).

Определим $\sigma_{*}(r)$ как функцию распределения “обратного экспоненциального распределения", т. е. положим

$$
\sigma_{*}(r)=\exp \left(-\frac{1}{r}\right), \quad r>0 .
$$

Как функция распределения расстояний, в дальнейшем $\sigma_{*}$ будет играть важную роль.

ОПРЕДЕЛЕНИЕ 2.9. Изотропный марковский оператор $P$, связанный с тройкой $\left(d, \mu, \sigma_{*}\right)$, будем называть стандартным марковским оператором, связанным с $(d, \mu)$.

Теорема 2.10. Пусть $d_{*} u \sigma_{*}$ определены равенствами (2.14) и (2.16).

(а) Тройки $(d, \mu, \sigma)$ u $\left(d_{*}, \mu, \sigma_{*}\right)$ индуцируют одинаковые изотропные марковские операторы.

(b) Ядро теплопроводности $p(t, x, y)$, связанное с тройкой $(d, \mu, \sigma)$, для всех $x, y \in X u t>0$ удовлетворяет следующим тождествам:

$$
p(t, x, y)=\int_{0}^{t / d_{*}(x, y)} N\left(x, \frac{s}{t}\right) e^{-s} d s
$$

$u$

$$
p(t, x, y)=t \int_{0}^{1 / d_{*}(x, y)} N(x, \tau) \exp (-\tau t) d \tau .
$$

Следовательно, $p(t, x, y)$ - конечная непрерывная функиия от $t, x, y$ для всех $t>0 u x \neq y$.

Как следует из (а), любой изотропный марковский оператор является в то же время стандартным марковским оператором, связанным с $\left(d_{*}, \mu\right)$. 


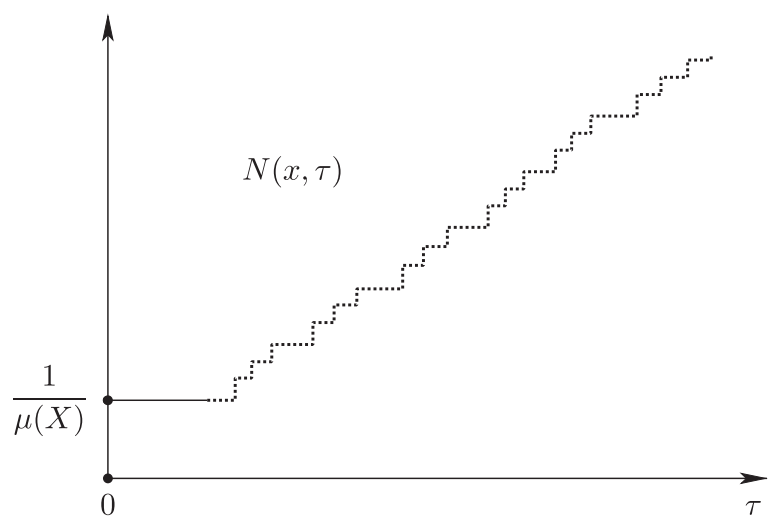

Рис. 1. График функции $\tau \mapsto N(x, \tau)$ в случае, когда $\mu(X)<\infty$

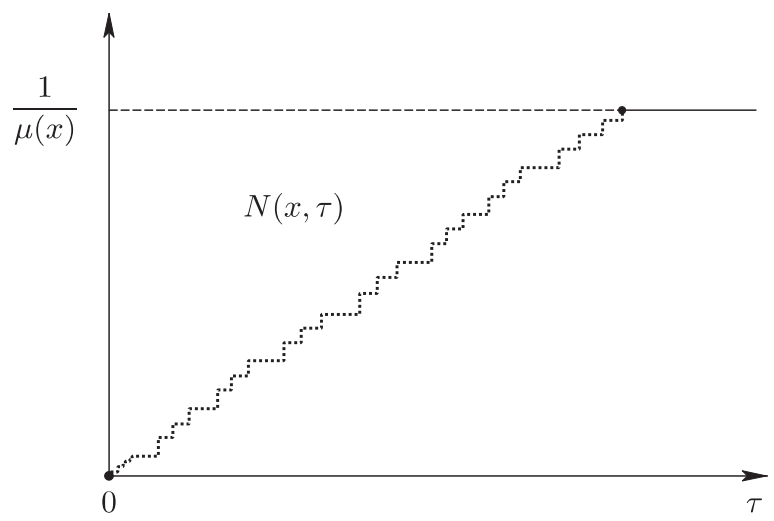

Рис. 2. График функции $\tau \mapsto N(x, \tau)$ в случае, когда $\mu(x)>0$

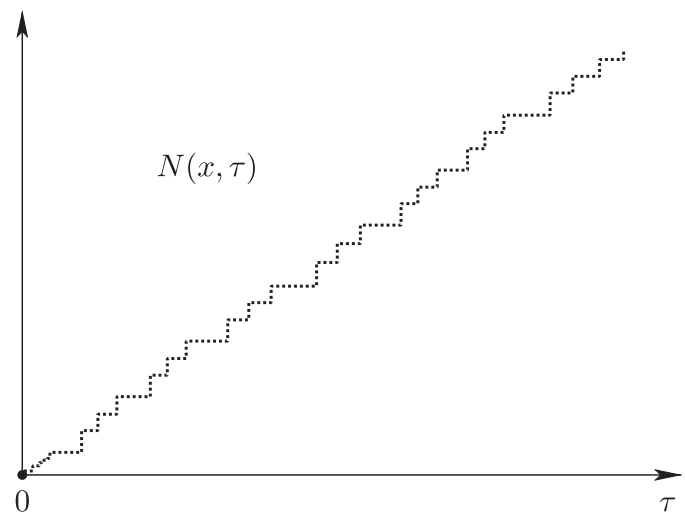

Рис. 3. График функции $\tau \mapsto N(x, \tau)$ в случае, когда $\mu(x)=0$ и $\mu(X)=\infty$ 
Доказательство. (а) Достаточно показать, что

$$
p(t, x, y)=\int_{\left[d_{*}(x, y), \infty\right)} \frac{d \sigma_{*}^{t}(u)}{\mu\left(B_{u}^{*}(x)\right)},
$$

где по теореме 2.2 правая часть представляет собой ядро теплопроводности, связанное с тройкой $\left(d_{*}, \mu, \sigma_{*}\right)$. Рассмотрим функцию

$$
u(r)=\frac{1}{\log (1 / \sigma(r))}, \quad r \in[0, \infty),
$$

и заметим, что

1) $u(d(x, y))=d_{*}(x, y), u(\infty)=\infty$;

2) $\sigma_{*}(u(r))=\exp (-1 / u(r))=\sigma(r)$;

3) $B_{u(r)}^{*}(x)=B_{r}(x)$ в силу леммы 2.7 .

Делая замену $u=u(r)$ в интеграле правой части (2.19), получаем

$$
\int_{\left[d_{*}(x, y), \infty\right)} \frac{d \sigma_{*}^{t}(u)}{\mu\left(B_{u}^{*}(x)\right)}=\int_{[d(x, y), \infty)} \frac{d \sigma^{t}(r)}{\mu\left(B_{r}(x)\right)},
$$

что вместе с (2.8) влечет (2.19). Ясно, что (2.18) следует из (2.17), так как $d_{*}(x, x)=0$.

(b) Замена $s=t / u$ в (2.19) дает

$$
\begin{aligned}
p(t, x, y) & =\int_{\left[d_{*}(x, y), \infty\right)} \frac{d \exp (-t / u)}{\mu\left(B_{u}^{*}(x)\right)} \\
& =\int_{t / d_{*}(x, y)}^{0} \frac{d e^{-s}}{\mu\left(B_{t / s}^{*}(x)\right)} \\
& =\int_{0}^{t / d_{*}(x, y)} N\left(x, \frac{s}{t}\right) e^{-s} d s,
\end{aligned}
$$

что доказывает (2.17). Еще одна замена $s=t \tau$ преобразует $(2.17)$ в (2.18).

Теорема доказана.

В случае $x=y$ из $(2.17)$ и (2.18) получаем

$$
p(t, x, x)=\int_{0}^{\infty} N\left(x, \frac{s}{t}\right) e^{-s} d s=t \int_{0}^{\infty} N(x, \tau) \exp (-\tau t) d \tau .
$$

В зависимости от функции $N(x, \tau)$ диагональное значение $p(t, x, x)$ может быть равно $\infty$. Для любого $x \in X$ положим

$$
T(x):=\limsup _{\tau \rightarrow \infty} \frac{\log N(x, \tau)}{\tau} .
$$

СлеДСтвиЕ 2.11. Функиия $t \mapsto p(t, x, x)$ монотонно убъвает, и для всех $t>T(x)$ выполнено неравенство $p(t, x, x)<\infty$.

ДокАЗАтЕЛЬство. Монотонность функции $p(t, x, x)$ следует из первого тождества в (2.20), в то время как второе утверждение следует из второго тождества в (2.20). Заметим также, что если предел $\lim _{\tau \rightarrow \infty} \frac{\log N(x, \tau)}{\tau}$ существует и потому равен $T(x)$, то $p(t, x, x)=\infty$ при $t<T(x)^{\tau}$. 
ПрЕДЛОЖЕНИЕ 2.12. Предположим, что $T(x)<\infty$ для некоторого $x \in X$.

(а) Для всех $y \in X$

$$
\lim _{t \rightarrow \infty} p(t, x, y)=\frac{1}{\mu(X)},
$$

где сходимость локально равномерна по $y \in X$.

(b) Для всех $y \in X$

$$
\lim _{t \rightarrow \infty} \frac{p(t, x, y)}{p(t, x, x)}=1,
$$

где сходимость локально равномерна по $y \in X$.

ДоказатеЛьство. (а) При $t \rightarrow \infty$ имеем

$$
N\left(x, \frac{s}{t}\right) \rightarrow N(x, 0)=\frac{1}{\mu(X)}
$$

и $t / d_{*}(x, y) \rightarrow \infty$. Отсюда и из (2.17) получим

$$
\lim _{t \rightarrow \infty} p(t, x, y)=\int_{0}^{\infty} \frac{1}{\mu(X)} e^{-s} d s=\frac{1}{\mu(X)},
$$

как только покажем, что интеграл и предел перестановочны. Последнее следует из теоремы о мажорируемой сходимости. Действительно, условие $T(x)<\infty$ влечет, что для некоторых $A, a>0$ и всех $\tau>0$

$$
N(x, \tau) \leqslant A \exp (a \tau)
$$

откуда

$$
N\left(x, \frac{s}{t}\right) e^{-s} \leqslant A \exp \left(\left(\frac{a}{t}-1\right) s\right) \leqslant A \exp \left(-\frac{1}{2} s\right)
$$

при $t>2 a$, так что условие мажорирования выполнено.

(b) Положим $r=d_{*}(x, y)$. Из (2.17) и (2.20) следует, что

$$
p(t, x, x)-p(t, x, y)=\int_{t / r}^{\infty} N\left(x, \frac{s}{t}\right) e^{-s} d s .
$$

Считая, что $t>2 a$, и применяя (2.23), получаем

$$
p(t, x, x)-p(t, x, y) \leqslant A \int_{t / r}^{\infty} e^{-s / 2} d s=2 A \exp \left(-\frac{t}{2 r}\right),
$$

в то время как

$$
p(t, x, x) \geqslant \int_{t /(4 r)}^{\infty} N\left(x, \frac{s}{t}\right) e^{-s} d s \geqslant N\left(x, \frac{1}{4 r}\right) \exp \left(-\frac{t}{4 r}\right) .
$$

Следовательно,

$$
\frac{p(t, x, x)-p(t, x, y)}{p(t, x, x)} \leqslant \frac{2 A \exp (-t /(4 r))}{N(x, 1 /(4 r))} \rightarrow 0 \quad \text { при } t \rightarrow \infty .
$$

Предложение доказано. 
2.4. Оценки ядра теплопроводности. Целью этого пункта является получение некоторых оценок изотропного ядра теплопроводности. Напомним, что по теореме 2.10

$$
p(t, x, y)=\int_{0}^{t / d_{*}(x, y)} N\left(x, \frac{s}{t}\right) e^{-s} d s .
$$

ОПРЕДЕЛЕНИЕ 2.13. Будем говорить, что монотонно возрастающая функция $\Phi: \mathbb{R}_{+} \rightarrow \mathbb{R}_{+}$удовлетворяют свойству удвоения, если существует такая постоянная $D>0$, что

$$
\Phi(2 s) \leqslant D \Phi(s) \quad \text { для всех } s>0 .
$$

Известно (теорема Поттера), что если $\Phi$ удовлетворяет свойству удвоения, то имеет место неравенство

$$
\Phi\left(s_{2}\right) \leqslant D\left(\frac{s_{2}}{s_{1}}\right)^{\gamma} \Phi\left(s_{1}\right) \quad \text { для всех } 0<s_{1}<s_{2}, \quad \text { где } \gamma=\log _{2} D .
$$

Теорема 2.14. Предположим, что для некоторого $x \in X$ функиия $\tau \mapsto$ $N(x, \tau)$ удовлетворяет свойству удвоения. Тогда

$$
\begin{aligned}
& \frac{c t}{t+d_{*}(x, y)} N\left(x, \frac{1}{t+d_{*}(x, y)}\right) \\
& \quad \leqslant p(t, x, y) \leqslant \frac{C t}{t+d_{*}(x, y)} N\left(x, \frac{1}{t+d_{*}(x, y)}\right)
\end{aligned}
$$

для всех $t>0, y \in X$ и некоторых постоянных $C, c>0$, зависящих от постоянной удвоения.

В дальнейшем мы будем использовать отношение $f \simeq g$ между двумя положительными функциями $f, g$, которое означает, что дробь $f / g$ ограничена сверху и снизу положительными постоянными для указанной области значений переменных. В частности, мы можем коротко записать (2.26) в виде

$$
p(t, x, y) \simeq \frac{t}{t+d_{*}(x, y)} N\left(x, \frac{1}{t+d_{*}(x, y)}\right)
$$

для фиксированного $x$ и всех $y \in X$ и $t>0$.

Пример 2.15. Предположим, что для некоторых $x \in X$ и $\alpha>0$

$$
N(x, \tau) \simeq \tau^{\alpha} \quad \text { для всех } \tau>0 .
$$

Тогда в силу (2.27) имеем

$$
p(t, x, y) \simeq \frac{t}{\left(t+d_{*}(x, y)\right)^{1+\alpha}} \simeq \frac{t}{\left(t^{2}+d_{*}(x, y)^{2}\right)^{(1+\alpha) / 2}},
$$

т. е. $p(t, x, y)$ ведет себя как распределение Коши в “ $\alpha$-мерном" пространстве. 
ПримеР 2.16. Более общо, предположим, что для некоторых $\alpha, \beta \geqslant 0$

$$
N(x, \tau) \simeq \begin{cases}\tau^{\alpha}, & 0<\tau \leqslant 1 \\ \tau^{\beta}, & \tau>1\end{cases}
$$

Тогда в силу (2.27) получаем

$$
p(t, x, y) \simeq \begin{cases}\frac{t}{\left(t+d_{*}(x, y)\right)^{1+\beta}}, & t+d_{*}(x, y) \leqslant 1 \\ \frac{t}{\left(t+d_{*}(x, y)\right)^{1+\alpha}}, & t+d_{*}(x, y)>1\end{cases}
$$

Например, пусть $X$ - дискретная локально конечная группа, например, $X=$ $\bigoplus_{k=1}^{\infty} \mathbb{Z}\left(n_{k}\right)$, а $\mu$ - мера Хаара, нормированная условием $\mu(x)=1$. С дискретной ультраметрикой получаем в силу (2.15), что $N(x, \tau) \simeq 1$ для достаточно больших $\tau$. Дополнительно предполагая, что

$$
N(x, \tau) \simeq \tau^{\alpha} \quad \text { для малых } \tau,
$$

видим, что (2.28), а потому и (2.29) выполняются с $\beta=0$ (ср. с [13]).

ПРимеР 2.17. Предположим, что функция $\tau \mapsto N(x, \tau)$ удовлетворяет свойству удвоения и для некоторого $\alpha>0$

$$
N(x, \tau) \simeq\left(\log \frac{1}{\tau}\right)^{-\alpha} \quad \text { при } \tau<\frac{1}{2} .
$$

Тогда в силу (2.27) имеем

$$
p(t, x, y) \simeq \frac{t}{\left(t+d_{*}(x, y)\right) \log ^{\alpha}\left(t+d_{*}(x, y)\right)},
$$

как только $t+d_{*}(x, y)>2$.

ПримеР 2.18. Предположим, что для некоторого $\alpha>0$

$$
N(x, \tau) \simeq \exp \left(-\tau^{-\alpha}\right)
$$

В этом случае теорема 2.14 не применима. Непосредственная оценка интеграла в (2.24) дает в этом случае

$$
p(t, x, y) \leqslant \frac{C_{3} t}{t+d_{*}(x, y)} \exp \left(-c_{3}\left(t^{\alpha /(\alpha+1)}+d_{*}(x, y)^{\alpha}\right)\right)
$$

и

$$
p(t, x, y) \geqslant \frac{C_{4} t}{t+d_{*}(x, y)} \exp \left(-c_{4}\left(t^{\alpha /(\alpha+1)}+d_{*}(x, y)^{\alpha}\right)\right)
$$

для всех $x, y \in X, t>0$ и некоторых положительных постоянных $C_{3}, C_{4}, c_{3}, c_{4}$. 
Для доказательства теоремы 2.14 мы используем несколько вспомогательных утверждений.

Лемма 2.19. Для всех $x, y \in X u t>0$ выполняются следующие оценки:

(a) $\quad p(t, x, y) \leqslant \frac{t}{d_{*}(x, y)} N\left(x, \frac{1}{d_{*}(x, y)}\right)$;

(b) $p(t, x, y) \geqslant \frac{1}{2 e} \begin{cases}\frac{t}{d_{*}(x, y)} N\left(x, \frac{1}{2 d_{*}(x, y)}\right), & t \leqslant d_{*}(x, y), \\ N\left(x, \frac{1}{2 t}\right), & t \geqslant d_{*}(x, y) ;\end{cases}$

(c) $\quad p(t, x, x) \geqslant \frac{1}{e} N\left(x, \frac{1}{t}\right)$.

ДокАзАтельство. (а) Неравенство (2.30) следует из (2.24) в силу монотонности функции $\tau \mapsto N(x, \tau)$, которая дает

$$
N\left(x, \frac{s}{t}\right) e^{-s} \leqslant N\left(x, \frac{1}{d_{*}(x, y)}\right)
$$

(b) Положим $a=\min \left(1, \frac{t}{d_{*}(x, y)}\right)$. Из $(2.24)$ следует, что

$$
p(t, x, y) \geqslant \int_{a / 2}^{a} N\left(x, \frac{s}{t}\right) e^{-s} d s \geqslant N\left(x, \frac{a}{2 t}\right) \frac{a}{2 e},
$$

что равносильно (2.31).

(c) В силу (2.20) имеем

$$
p(t, x, x) \geqslant \int_{1}^{\infty} N\left(x, \frac{s}{t}\right) e^{-s} d s \geqslant N\left(x, \frac{1}{t}\right) \int_{1}^{\infty} e^{-s} d s,
$$

откуда следует (2.32).

Лемма доказана.

Лемма 2.20. Следующие неравенства выполняются для всех $x, y \in X u$ $t>0$ :

$$
p(t, x, y) \geqslant \frac{1}{2 e} \frac{t}{t+d_{*}(x, y)} N\left(x, \frac{1}{2\left(t+d_{*}(x, y)\right)}\right)
$$

$u$

$$
p(t, x, y) \leqslant 2 e \frac{t}{t+d_{*}(x, y)} p\left(\frac{t+d_{*}(x, y)}{2}, x, x\right) .
$$


ДокАзАтельство. Нижняя оценка (2.33) сразу следует из (2.31). Чтобы доказать (2.34), заметим, что в силу (2.30) и (2.32) выполняется неравенство

$$
p(t, x, y) \leqslant e \frac{t}{d_{*}(x, y)} p\left(d_{*}(x, y), x, x\right),
$$

что дает $(2.34)$ в случае $t \leqslant d_{*}(x, y)$, так как функция $p(\cdot, x, x)$ монотонно убывает. В случае $t>d_{*}(x, y)$ неравенство (2.34) тривиально следует из (2.12), т. е. из

$$
p(t, x, y) \leqslant p(t, x, x)
$$

снова с использованием монотонности $p(\cdot, x, x)$. Лемма доказана.

Лемма 2.21. Для любого заданного $x \in X$ следующие два свойства равносильны:

(i) для некоторой постоянной $C$ и всех $t>0$

$$
p(t, x, x) \leqslant C N\left(x, \frac{1}{t}\right)
$$

(ii) функиия $\tau \mapsto N(x, \tau)$ удовлетворяет свойству удвоения, т.е. для некоторой постоянной $D$

$$
N(x, 2 \tau) \leqslant D N(x, \tau)
$$

Доказательство. (ii) $\Rightarrow$ (i). Оценка (2.35) вытекает из (2.20) и (2.25) следующим образом:

$$
\begin{aligned}
p(t, x, x) & =N\left(x, \frac{1}{t}\right) \int_{0}^{\infty} \frac{N(x, s / t)}{N(x, 1 / t)} e^{-s} d s \\
& \leqslant D N\left(x, \frac{1}{t}\right) \int_{0}^{\infty} \max \left\{1, s^{\gamma}\right\} e^{-s} d s \\
& =C N\left(x, \frac{1}{t}\right) .
\end{aligned}
$$

(i) $\Rightarrow$ (ii). Из верхней оценки (2.35) следует, что для любого $t>0$

$$
\begin{aligned}
C N\left(x, \frac{1}{t}\right) & \geqslant p(t, x, x) \geqslant \int_{2}^{\infty} N\left(x, \frac{s}{t}\right) e^{-s} d s \\
& \geqslant e^{-2} N\left(x, \frac{2}{t}\right),
\end{aligned}
$$

откуда вытекает свойство удвоения функции $N(x, \cdot)$.

Лемма доказана.

ДокАЗАТЕЛЬСтво тЕОРЕмы 2.14. Нижняя оценка в (2.26) следует из (2.33), верхняя оценка следует из (2.34) и (2.35). Теорема доказана. 
В заключение этого пункта приведем достаточные условия для справедливости свойства удвоения функции $N(x, \cdot)$.

ОПРЕДЕЛЕНИЕ 2.22. Будем говорить, что монотонно возрастающая функция $\Psi: \mathbb{R}_{+} \rightarrow \mathbb{R}_{+}$удовлетворяют обратному свойству удвоения, если имеется такая постоянная $\delta \in(0,1)$, что для всех $r>0$ выполнено неравенство

$$
\Psi(r) \geqslant 2 \Psi(\delta r)
$$

ПреДложениЕ 2.23. Зафиксируем некоторое $x \in X$. Функиия $\tau \mapsto N(x, \tau)$ удовлетворяет свойству удвоения, если выполняются следующие два условия:

(i) бункиия $\Psi(r)=-1 / \log \sigma(r)$ удовлетворяет обратному свойству удвоения;

(ii) функиия объема $r \mapsto \mu\left(B_{r}(x)\right)$ удовлетворяет свойству удвоения.

ДокАзАтельство. Мы используем следующие сокращенные обозначения для шаров с центром в $x: B_{r}=B_{r}(x)$ и $B_{r}^{*}=B_{r}^{*}(x)$. Из определения 2.8 спектральной функции следует, что функция $\tau \mapsto N(x, \tau)$ удовлетворяет свойству удвоения тогда и только тогда, когда функция $s \mapsto \mu\left(B_{s}^{*}\right)$ удовлетворяет этому свойству. Положим $\Phi=\Psi^{-1}$ и заметим, что обратное свойство удвоения для $\Psi$ равносильно свойству удвоения для $\Phi$. В силу леммы 2.7 имеем $B_{\Psi(r)}^{*}=B_{r}$, откуда следует, что $B_{s}^{*}=B_{\Phi(s)}$. Используя условия (ii) и $(2.25)$ для функции $\mu\left(B_{r}\right)$, получаем

$$
\mu\left(B_{2 s}^{*}\right)=\mu\left(B_{\Phi(2 s)}\right) \leqslant D\left(\frac{\Phi(2 s)}{\Phi(s)}\right)^{\gamma} \mu\left(B_{\Phi(s)}\right) \leqslant \operatorname{const} \mu\left(B_{s}^{*}\right),
$$

что и требовалось доказать.

2.5. Тепловые ядра в $\mathbb{Q}_{p}$. Пусть $p$ - простое число, тогда $p$-адическая норма на множестве рациональных чисел $\mathbb{Q}$ определяется так: если $x=p^{n} a / b$, где $a, b$ - целые числа, не делящиеся на $p$, то

$$
\|x\|_{p}:=p^{-n}
$$

Если $x=0$, то $\|x\|_{p}:=0$. Для $p$-адической нормы на $\mathbb{Q}$ имеет место ультраметрическое неравенство. Действительно, если $y=p^{m} c / d$ и $m \leqslant n$, то

$$
x+y=p^{m}\left(\frac{p^{n-m} a}{b}+\frac{c}{d}\right),
$$

откуда

$$
\|x+y\|_{p} \leqslant p^{-m}=\max \left\{\|x\|_{p},\|y\|_{p}\right\} .
$$

Отсюда следует, что $\mathbb{Q}$ с метрикой $d(x, y)=\|x-y\|_{p}$ является ультраметрическим пространством, и таковым же является его пополнение $\mathbb{Q}_{p}-$ поле $p$-адических чисел.

Каждое $p$-адическое число $x$ имеет представление

$$
x=\sum_{k=-N}^{\infty} a_{k} p^{k}=\ldots a_{k} \ldots a_{2} a_{1} a_{0} \cdot a_{-1} a_{-2} \ldots a_{-N},
$$


где $N \in \mathbb{N}$, а $a_{k} \in\{0, \ldots, p-1\}-p$-адические цифры. Рациональное число $0 . a_{-1} \ldots a_{-N}=\sum_{k=-N}^{-1} a_{k} p^{k}$ называется дробной частью $x$, а остальное, $\sum_{k=0}^{\infty} a_{k} p^{k},-$ целой частью $x$.

Для любого $n \in \mathbb{Z}$ определим $d$-шар $B_{p^{-n}}(x):$ он состоит из всех таких чисел

$$
y=\sum_{k=-N}^{\infty} b_{k} p^{k}=\ldots b_{k} \ldots b_{2} b_{1} b_{0} \cdot b_{-1} b_{-2} \ldots b_{-N}
$$

что $b_{k}$ произвольны при $k \geqslant n$ и $b_{k}=a_{k}$ при $k<n$. Следовательно, $B_{p^{-n}}(x)$ разлагается в объединение $p$ непересекающихся шаров радиусов $p^{-(n+1)}$, зависящих от выбора $b_{n}$.

Например, $B_{1}(0)$ совпадает с множеством $\mathbb{Z}_{p}$ всех целых $p$-адических чисел, т. е. любое $y \in B_{1}(0)$ имеет вид

$$
y=\ldots b_{k} \ldots b_{2} b_{1} b_{0}
$$

с произвольными $p$-адическими цифрами $b_{k}$. Для любого фиксированного $c \in$ $\{0,1, \ldots, p-1\}$ дополнительное ограничение $b_{0}=c$ определяет шар радиуса $1 / p$ с центром в $c$, так что $B_{1}(0)$ является объединением $p$ таких непересекающихся шаров, как на следующей диаграмме, где каждая клетка изображает один из шаров $B_{1 / p}(c)$ :

\begin{tabular}{|l|l|l|l|}
\hline$\ldots b_{k} \ldots b_{2} b_{1} 0$ & $\ldots b_{k} \ldots b_{2} b_{1} 1$ & $\ldots$ & $\ldots b_{k} \ldots b_{2} b_{1}(p-1)$ \\
\hline
\end{tabular}

Пусть $\mu$ - аддитивная мера Хаара на $\mathbb{Q}_{p}$, нормированная таким образом, что $\mu\left(B_{1}(0)\right)=1$. Поскольку

$$
B_{r}(x)=x+B_{r}(0)
$$

и $\mu$ инвариантна относительно сдвигов, получаем, что $\mu\left(B_{r}(x)\right)$ не зависит от $x$. Вышеприведенное рассуждение с разложением шара $B_{p^{-n}}(x)$ показывает, что

$$
\mu\left(B_{p^{-n}}(x)\right)=p \mu\left(B_{p^{-(n+1)}}(x)\right)
$$

откуда следует равенство

$$
\mu\left(B_{p^{-n}}(x)\right)=p^{-n}
$$

Для любого $r>0$ шар $B_{r}(x)$ совпадает с $B_{p^{-n}}(x)$, где $n \in \mathbb{Z}$ таково, что $p^{-n} \leqslant$ $r<p^{-(n-1)}$, откуда следует, что для всех $r>0$

$$
\frac{r}{p}<\mu\left(B_{r}(x)\right) \leqslant r
$$


ПримеР 2.24. Пусть $(X, d, \mu)$ есть $\mathbb{Q}_{p}$ с $p$-адическим расстоянием и мерой Хаара $\mu$. Рассмотрим функцию распределения расстояний

$$
\sigma(r)=\exp \left(-\left(\frac{b}{r}\right)^{\alpha}\right)
$$

где $\alpha, b>0$. Поскольку

$$
\Psi(r):=\frac{1}{\log (1 / \sigma(r))}=\left(\frac{r}{b}\right)^{\alpha}
$$

в силу (2.14) получаем

$$
d_{*}(x, y)=\Psi(d(x, y))=\left(\frac{\|x-y\|_{p}}{b}\right)^{\alpha} .
$$

В силу леммы 2.7 имеем

$$
B_{s}^{*}(x)=B_{\Psi^{-1}(s)}(x),
$$

что вместе с (2.38) дает

$$
\mu\left(B_{s}^{*}(x)\right) \simeq s^{1 / \alpha}
$$

Следовательно,

$$
N(x, \tau) \simeq \tau^{1 / \alpha}
$$

Поскольку эта функция удовлетворяет свойству удвоения, теорема 2.14 (ср. также с примером 2.15) дает оценку

$$
p(t, x, y) \simeq \frac{t}{\left(t+d_{*}(x, y)\right)^{1+1 / \alpha}} \simeq \frac{t}{\left(t^{1 / \alpha}+\|x-y\|_{p}\right)^{1+\alpha}} .
$$

В частности, для всех $t>0$ и $x \in X$ имеем

$$
p(t, x, x) \simeq t^{1 / \alpha}
$$

Пример 2.25. Пусть $X=\mathbb{Z}_{p}$, т. е. $X$ - единичный шар $B_{1}(0)$ в $\mathbb{Q}_{p}$ с $p$-адическим расстоянием и мерой Хаара $\mu$. Рассмотрим функцию распределения расстояний

$$
\sigma(r)=\exp \left(1-\exp \left(r^{-\alpha}\right)\right)
$$

для некоторого $\alpha>0$. Поскольку при $r \leqslant 1$

$$
\Psi(r):=\frac{1}{\log (1 / \sigma(r))}=\frac{1}{\exp \left(r^{-\alpha}\right)-1} \simeq \exp \left(-r^{-\alpha}\right),
$$

получаем, что

$$
d_{*}(x, y)=\Psi(d(x, y)) \simeq \exp \left(-\|x-y\|_{p}^{-\alpha}\right)
$$


В силу (2.38) и леммы 2.7 для всех $s \leqslant 1 / 2$ имеем

$$
\mu\left(B_{s}^{*}(x)\right)=\mu\left(B_{\Psi^{-1}(s)}(x)\right) \simeq \Psi^{-1}(s) \simeq \frac{1}{\log ^{1 / \alpha}(1 / s)},
$$

в то время как при $s>1 / 2$ имеем $\mu\left(B_{s}^{*}(x)\right) \simeq 1$. Поэтому для всех $\tau>0$

$$
N(x, \tau)=\frac{1}{\mu\left(B_{1 / \tau}^{*}(x)\right)} \simeq \log ^{1 / \alpha}(2+\tau) .
$$

Отсюда следует, что функция $N(x, \tau)$ удовлетворяет свойству удвоения, и мы получаем в силу (2.27), что

$$
p(t, x, y) \simeq \frac{t}{t+\exp \left(-\|x-y\|_{p}^{-\alpha}\right)} \log ^{1 / \alpha}\left(2+\frac{1}{t+\exp \left(-\|x-y\|_{p}^{-\alpha}\right)}\right) .
$$

Пример 2.26. Пусть $X-$ подмножество из $\mathbb{Q}_{p}$, состоящее из всех $p$-адических дробей, т. е. чисел вида $x=0 . a_{-1} \ldots a_{-N}$. Тогда $p$-адическое расстояние $d$ на $X$ принимает только целые значения, так что $(X, d)$ - дискретное пространство. Пусть $\mu$ - считающая мера на $X$, т.е. $\mu(x)=1$ для любого $x \in X$. Рассмотрим следующую функцию распределения расстояний:

$$
\sigma(r)=\exp \left(-\frac{1}{\log ^{\alpha}(2 r)}\right) \quad \text { при } r \geqslant 1,
$$

которая произвольным образом продолжена на $r<1$ так, чтобы быть строго монотонно возрастающей и удовлетворять $\sigma(0)=0$. Поскольку

$$
\Psi(r):=\frac{1}{\log (1 / \sigma(r))}=\log ^{\alpha}(2 r) \quad \text { при } r \geqslant 1,
$$

при $x \neq y$ получаем

$$
d_{*}(x, y)=\Psi(d(x, y))=\log ^{\alpha}\left(2\|x-y\|_{p}\right) .
$$

При $s \geqslant s_{0}:=\log ^{\alpha} 2$ имеем

$$
\mu\left(B_{s}^{*}(x)\right)=\mu\left(B_{\Psi^{-1}(s)}(x)\right) \simeq \Psi^{-1}(s)=\frac{1}{2} \exp \left(s^{1 / \alpha}\right),
$$

в то время как при $s<s_{0}$ имеем $\mu\left(B_{s}^{*}(x)\right) \simeq \mu(x)=1$. Видим, что $(2.43)$ выполняется для всех $s>0$. Следовательно, для всех $\tau>0$

$$
N(x, \tau)=\frac{1}{\mu\left(B_{1 / \tau}^{*}(x)\right)} \simeq \exp \left(-\tau^{-1 / \alpha}\right) .
$$

В силу примера 2.18 получаем

$$
p(t, x, y) \leqslant \frac{C t}{t+\log _{+}^{\alpha}\left(2\|x-y\|_{p}\right)} \exp \left(-c\left(t^{1 /(\alpha+1)}+\log _{+}\left(2\|x-y\|_{p}\right)\right)\right),
$$

а также аналогичную нижнюю оценку. 
2.6. Функция Грина и невозвратность. Для заданной изотропной полугруппы $\left\{P^{t}\right\}$ определим оператор Грина $G$ на неотрицательных борелевских функциях $f$ на $X$ формулой

$$
G f(x)=\int_{0}^{\infty} P^{t} f(x) d t .
$$

Конечно, значение $G f(x)$ может быть равно $\infty$. По теореме Фубини получаем

$$
G f(x)=\int_{X} g(x, y) f(y) d \mu(y)
$$

где

$$
g(x, y)=\int_{0}^{\infty} p(t, x, y) d t
$$

Подставляя ядро теплопроводности из (2.18) и снова используя теорему Фубини, получаем

$$
g(x, y)=\int_{0}^{1 / d_{*}(x, y)} \frac{N(x, \tau) d \tau}{\tau^{2}}=\int_{d_{*}(x, y)}^{\infty} \frac{d s}{\mu\left(B_{s}^{*}(x)\right)},
$$

где второе тождество следует из (2.15). Функция $g(x, y)$ называется функиией Грина полугруппы $\left\{P^{t}\right\}$. Заметим, что функция Грина может быть тождественно равна $\infty$. Например, это так, когда $\mu(x)<\infty$ (ср. с рис. 1$)$ и второй интеграл в (2.45) расходится на $\infty$.

ОПРЕДЕлЕниЕ 2.27. Процесс $\left\{\mathscr{X}_{t}\right\}$ и полугруппа $\left\{P^{t}\right\}$ называются невозвратными, если $G f$ - ограниченная функция, как только $f$ ограничена и имеет компактный носитель, и возвратными в противном случае.

Теорема 2.28. Следующие утверждения равносильны:

(i) полугруппа $\left\{P^{t}\right\}$ является невозвратной;

(ii) $g(x, y)<\infty$ для некоторых/всех различных $x, y \in X$;

(iii) для некоторого/всех $x \in X$

$$
\int^{\infty} \frac{d s}{\mu\left(B_{s}^{*}(x)\right)}<\infty .
$$

Неравенство (2.46) равносильно неравенству

$$
\int_{0} \frac{N(x, \tau) d \tau}{\tau^{2}}<\infty
$$

Заметим, что в невозвратном случае функция $x, y \mapsto 1 / g(x, y)$ определяет ультраметрику на $X$, что доказывается аналогично следствию 2.6.

ДокАзАтельство. Справедливость условия (2.46) не зависит от выбора $x$, поскольку для любых двух $x, x^{\prime} \in X$ шары $B_{s}^{*}(x)$ и $B_{s}^{*}\left(x^{\prime}\right)$ совпадают, как только $s \geqslant d\left(x, x^{\prime}\right)$. Ясно, что конечность второго интеграла в (2.45) при $x \neq y$ равносильна (2.46), откуда следует эквивалентность (ii) $\Leftrightarrow$ (iii), причем со всеми комбинациями вариантов некоторых/всех. 
Ясно, что из конечности $G f$ для любой ограниченной функции $f$ с компактным носителем вытекает, что $g(x, y) \not \equiv \infty$, т. е. (i) $\Rightarrow$ (ii). Итак, остается доказать импликацию (iii) $\Rightarrow$ (i). Достаточно показать, что $G f$ ограничена для $f=\mathbf{1}_{A}$, где $A$ - ограниченное борелевское подмножество из $X$. Пусть $R$ - диаметр множества $A$ относительно расстояния $d^{*}$. Тогда имеем $A \subset B_{R}^{*}(x)$ для любого $x \in A$, откуда в силу (2.45) получаем

$$
\begin{aligned}
G f(x) & =\int_{A} g(x, y) d \mu(y) \leqslant \int_{B_{R}^{*}(x)} g(x, y) d \mu(y) \\
& =\int_{B_{R}^{*}(x)} \int_{0}^{\infty} \mathbf{1}_{\left[d_{*}(x, y), \infty\right)}(s) \frac{d s}{\mu\left(B_{s}^{*}(x)\right)} d \mu(y) \\
& =\int_{0}^{\infty} \frac{1}{\mu\left(B_{s}^{*}(x)\right)}\left(\int_{B_{R}^{*}(x)} \mathbf{1}_{[0, s]}\left(d_{*}(x, y)\right) d \mu(y)\right) d s \\
& =\int_{0}^{\infty} \frac{1}{\mu\left(B_{s}^{*}(x)\right)} \mu\left(B_{R}^{*}(x) \cap B_{s}^{*}(x)\right) d s .
\end{aligned}
$$

Для $s \geqslant R$ подынтегральная функция равна $\frac{1}{\mu\left(B_{s}^{*}(x)\right)} \mu\left(B_{R}^{*}(x)\right)$, так что сходимость на $\infty$ следует из (2.46). Ясно, что сходимость равномерна по $x \in A$, поскольку $\mu\left(B_{R}^{*}(x)\right)$ и $\mu\left(B_{s}^{*}(x)\right)$ не зависят от $x \in A$ при $s \geqslant R$. Для $s \leqslant R$ подынтегральная функция равна

$$
\frac{1}{\mu\left(B_{s}^{*}(x)\right)} \mu\left(B_{s}^{*}(x)\right)=1
$$

что влечет за собой равномерную сходимость в нуле. Отсюда вытекает, что $\sup _{A} G f(x)<\infty$. Наконец, соотношение $\sup _{X} G f(x)<\infty$ следует из того, что $g(x, y)$ убывает как функция от $d_{*}(x, y)$. Теорема доказана.

Отметим, что если $X$ - локально конечная группа с мерой Хаара $\mu$, то критерий невозвратности (iii) теоремы 2.28 совпадает с общим достаточным условием невозвратности из [40].

Теперь дадим некоторые оценки функции Грина. Положим

$$
V(x, r)=\mu\left(B_{r}^{*}(x)\right) .
$$

ТеОРема 2.29. Предположим, что существуют такие постоянные $1<$ $c<c^{\prime}<c^{\prime \prime}$, что для всех $r>r_{0} \geqslant 0$ и некоторого $x \in X$

$$
c^{\prime} \leqslant \frac{V(x, c r)}{V(x, r)} \leqslant c^{\prime \prime}
$$

Тогда полугруппа $\left\{P^{t}\right\}$ невозвратна и для всех $y \in X$, для которьх $r:=$ $d^{*}(x, y)>r_{0}$, имеем

$$
g(x, y) \simeq \frac{r}{V(x, r)} .
$$

Заметим, что условие $\frac{V(x, c r)}{V(x, r)} \leqslant c^{\prime \prime}$ равносильно свойству удвоения функции $r \mapsto V(x, r)$ (ср. с определением 2.13), в то время как условие $\frac{V(x, c r)}{V(x, r)} \geqslant c^{\prime}$ при 
$c^{\prime}>c$ несколько сильнее обратного свойства удвоения (ср. с определением 2.22). Например, (2.49) выполняется при $V(x, r) \simeq r^{\alpha}$ тогда и только тогда, когда $\alpha>1$.

ДокАЗАТЕЛЬСтво. Для простоты обозначений положим $V(s):=V(x, s)$. При $r>r_{0}$ имеем

$$
g(x, y)=\int_{r}^{\infty} \frac{d s}{V(s)}=\sum_{k=0}^{\infty} \int_{c^{k} r}^{c^{k+1} r} \frac{d s}{V(s)}=\sum_{k=0}^{\infty} c^{k} \int_{r}^{c r} \frac{d s}{V\left(c^{k} s\right)} .
$$

Используя нижнюю оценку в (2.49), получаем

$$
\int_{r}^{\infty} \frac{d s}{V(s)} \leqslant \sum_{k=0}^{\infty} c^{k} \int_{r}^{c r} \frac{\left(c^{\prime}\right)^{-k} d s}{V(s)} \leqslant \sum_{k=0}^{\infty}\left(\frac{c}{c^{\prime}}\right)^{k} \frac{c r}{V(r)} \leqslant \text { const } \frac{r}{V(r)},
$$

где ряд сходится благодаря условию $c^{\prime}>c$. Аналогично, используя верхнюю оценку в (2.49), получаем

$$
\int_{r}^{\infty} \frac{d s}{V(s)} \geqslant \int_{r}^{c r} \frac{d s}{V(s)} \geqslant \frac{(c-1) r}{V(c r)} \geqslant \operatorname{const} \frac{r}{V(r)},
$$

что завершает доказательство теоремы.

Пример 2.30. Пусть $(X, d, \mu)$ и $\sigma$ - такие, как в примере 2.24 , т. е. $X=$ $\mathbb{Q}_{p}$ - поле $p$-адических чисел с ультраметрикой $d(x, y)=\|x-y\|_{p}$, а $\sigma(r)=$ $\exp \left(-(b / r)^{\alpha}\right)$. Тогда в силу $(2.39)$ имеем

$$
d_{*}(x, y)=\text { const }\|x-y\|_{p}^{\alpha},
$$

а в силу (2.40) имеем

$$
V(x, r) \simeq r^{1 / \alpha}
$$

Поэтому по теореме 2.28 полугруппа $\left\{P^{t}\right\}$ будет невозвратной тогда и только тогда, когда $\alpha<1$. Более того, условие (2.49) также выполняется тогда и только тогда, когда $\alpha<1$, и в этом случае мы получаем по теореме 2.29 , что для всех $x, y$

$$
g(x, y) \simeq d_{*}(x, y)^{1-1 / \alpha} \simeq\|x-y\|_{p}^{\alpha-1}
$$

Пример 2.31. Пусть $(X, d, \mu)$ и $\sigma$ - такие, как в примере 2.26 , т. е. $X$ - множество дробных $p$-адических чисел, а $\sigma$ задана равенством (2.41). В силу $(2.42)$ при $x \neq y$ имеем

$$
d_{*}(x, y)=\log ^{\alpha}\left(2\|x-y\|_{p}\right),
$$

а в силу (2.43) имеем

$$
V(x, r) \simeq \exp \left(r^{1 / \alpha}\right)
$$

По теореме 2.28 заключаем, что полугруппа $\left\{P^{t}\right\}$ является невозвратной. Теорема 2.29 в этом случае не применима, но, оценивая напрямую интеграл 
в $(2.45)$, мы получим для $r:=d^{*}(x, y)$

$$
g(x, y)=\int_{r}^{\infty} \frac{d s}{V(x, s)} \simeq \int_{r}^{\infty} \exp \left(-s^{1 / \alpha}\right) d s \simeq r^{1-1 / \alpha} \exp \left(-r^{1 / \alpha}\right),
$$

откуда при $x \neq y$ следует

$$
g(x, y) \simeq\|x-y\|_{p}^{-1} \log ^{\alpha-1}\left(2\|x-y\|_{p}\right) .
$$

\section{3. Изотропный лапласиан и его спектр}

В этом разделе мы рассматриваем свойства генератора изотропной полугруппы $\left\{P^{t}\right\}$. По определению генератор $\mathscr{L}$ сильно непрерывной полугруппы $\left\{P^{t}\right\}_{t \geqslant 0}$ в банаховом пространстве определяется равенством

$$
\mathscr{L} f=s-\lim _{t \rightarrow 0} \frac{f-P^{t} f}{t},
$$

причем область определения $\operatorname{dom}_{\mathscr{L}}$ состоит из тех $f$, для которых этот предел существует. Поскольку изотропная полугруппа $\left\{P^{t}\right\}$ симметрична и действует в гильбертовом пространстве $L^{2}(X, \mu)$, определение, приведенное выше, равносильно следующему: $\mathscr{L}$ - самосопряженный (неограниченный) оператор в $L^{2}(X, \mu)$ такой, что

$$
P^{t}=\exp (-t \mathscr{L}) \quad \text { для всех } t>0 \text {. }
$$

Очевидно, что это равносильно равенству $P=\exp (-\mathscr{L})$, что приводит к тождеству

$$
\mathscr{L}=\log \frac{1}{P},
$$

где правая часть понимается в смысле функционального исчисления самосопряженных операторов. Мы называем $\mathscr{L}$ изотропным оператором Лапласа, связанным с триплетом $(d, \mu, \sigma)$.

3.1. Субординация. Используя спектральное разложение (2.10) оператора $P$, получаем, что

$$
\mathscr{L}=\int_{[0,+\infty)} \log \frac{1}{\sigma(1 / \lambda)} d E_{\lambda}
$$

где $\left\{E_{\lambda}\right\}$ - разложение единицы, задаваемое формулой (2.6). Для простоты обозначим

$$
\varphi(\lambda):=\log \frac{1}{\sigma(1 / \lambda)}
$$

так что

$$
\mathscr{L}=\int_{[0,+\infty)} \varphi(\lambda) d E_{\lambda} .
$$

Область определения $\operatorname{dom}_{\mathscr{L}}$ тогда задается равенством

$$
\operatorname{dom}_{\mathscr{L}}=\left\{f \in L^{2}: \int_{0}^{\infty} \varphi(\lambda)^{2} d\left(E_{\lambda} f, f\right)<\infty\right\} .
$$


Заметим, что функция $\varphi$ обладает следующими свойствами, которые вытекают из предположений (1.5) о функции $\sigma$ :

\section{$\varphi:[0, \infty] \rightarrow[0, \infty]$ является строго монотонно возрастающей}

непрерывной справа функиией такой, что $\varphi(0)=0$ и $\varphi(\infty-)=\infty$.

Обратно, любая функция $\varphi$, удовлетворяющая (3.3), определяет функцию

$$
\sigma(\lambda)=\exp \left(-\varphi\left(\frac{1}{\lambda}\right)\right)
$$

которая удовлетворяет (1.5). Это наблюдение приводит к следующему интересному свойству субординации изотропных лапласианов.

Теорема 3.1. Пусть $\mathscr{L}$ - изотропный лапласиан, связанный $c(d, \mu, \sigma)$. Пусть $\psi$ - любая функция, удовлетворяющая (3.3). Тогда $\psi(\mathscr{L})$ также является изотропным лапласианом, связанным $c(d, \mu, \widetilde{\sigma})$, для некоторой другой функиии распределения расстояний $\widetilde{\sigma}$.

ДокАзАтЕЛЬСтво. Из (3.2) следует, что

$$
\psi(\mathscr{L})=\int_{[0,+\infty)} \psi \circ \varphi(\lambda) d E_{\lambda} .
$$

Поскольку композиция $\psi$ ○ также удовлетворяет $(3.3)$, получаем, что $\psi(\mathscr{L})$ является изотропным лапласианом. Более того, используя (3.1), мы получаем следующую формулу для $\widetilde{\sigma}$ :

$$
\tilde{\sigma}(r)=\exp \left(-\psi\left(\log \frac{1}{\sigma(r)}\right)\right) .
$$

Теорема доказана.

ЗАмЕчАнИЕ 3.2. Любой неотрицательно определенный самосопряженный оператор $\mathscr{L}$ в $L^{2}$ порождает полугруппу $\left\{e^{-t \mathscr{L}}\right\}_{t \geqslant 0}$. Мы называем $\mathscr{L}$ лапласианом, если полугруппа $\left\{e^{-t \mathscr{L}}\right\}$ марковская. В общем случае по теореме Бохнера для любого лапласиана $\mathscr{L}$ оператор $\psi(\mathscr{L})$ снова является лапласианом, как только $\psi$ является функцией Бернштейна (см., например, книгу Р. Л. Шиллинга, Р. Сонга и 3. Вондрачека [53]). Известно, что $\psi(\lambda)=\lambda^{\alpha}$ является функцией Бернштейна тогда и только тогда, когда $0<\alpha \leqslant 1$. Таким образом, для лапласиана общего вида $\mathscr{L}$ степень $\mathscr{L}^{\alpha}$ гарантированно является лапласианом только при $\alpha \leqslant 1$. Например, для классического оператора Лапласа $\mathscr{L}=-\Delta$ в $\mathbb{R}^{n}$ степень $(-\Delta)^{\alpha}$ при $\alpha>1$ не является лапласианом. В поразительном контрасте с этим фактом, по теореме 3.1 степени $\mathscr{L}^{\alpha}$ изотропного лапласиана снова являются лапласианами для всех $\alpha>0$.

3.2. $L^{2}$-спектр. Нашей следующей целью является вывод явного выражения для $\mathscr{L} f$ и описание спектра оператора $\mathscr{L}$. Напомним, что по теореме 2.10 тройки $(d, \mu, \sigma)$ и $\left(d_{*}, \mu, \sigma_{*}\right)$ индуцируют один и тот же марковский оператор $P$ 
и потому один и тот же оператор Лапласа $\mathscr{L}$, где $d_{*}-$ внутренняя ультраметрика, определенная равенством (2.14), а

$$
\sigma_{*}(r)=\exp \left(-\frac{1}{r}\right) .
$$

С этого момента мы будем использовать только метрику $d_{*}$ и $\sigma_{*}$. Пусть разложение единицы $\left\{E_{\lambda}\right\}$ также будет определено с использованием метрики $d_{*}$; это означает, что в определении (2.6) оператора $E_{\lambda}$ мы теперь используем усредняющий оператор $\mathrm{Q}_{r}$ относительно метрики $d_{*}$. Функция $\varphi_{*}$, связанная с $\sigma_{*}$ с помощью (3.1), имеет особенно простой вид: $\varphi_{*}(\lambda)=\lambda$. Поэтому мы получаем из (3.2) спектральное разложение оператора $\mathscr{L}$ в классическом виде

$$
\mathscr{L}=\int_{[0,+\infty)} \lambda d E_{\lambda}=\int_{(0, \infty)} \lambda d E_{\lambda} .
$$

Замена $s=1 / \lambda$ дает

$$
\mathscr{L}=-\int_{(0, \infty)} \frac{1}{s} d \mathrm{Q}_{s} .
$$

Для любого $x \in X$ обозначим через $\Lambda(x)$ множество значений $d_{*}(x, y)$ для всех $y \in X, y \neq x$, т. е.

$$
\Lambda(x)=\{d(x, y): y \in X \backslash\{x\}\}
$$

Лемма 3.3. Множество $\Lambda(x)$ не имеет точек сгущения в $(0, \infty)$. Следовательно, $\Lambda(x)$ не более чем счетно.

ДокАЗАтельство. Пусть $r \in(0, \infty)$ - точка сгущения множества $\Lambda(x)$, т. е. имеется последовательность $\left\{r_{k}\right\}$ точек из $\Lambda(x) \backslash\{r\}$, для которой $r_{k} \rightarrow r$ при $k \rightarrow \infty$. Тогда $r_{k}=d_{*}\left(x, y_{k}\right)$ для некоторого $y_{k} \in X$. Поскольку последовательность $\left(y_{k}\right)$ ограничена, в силу компактности всех шаров в $X$ она обладает сходящейся подпоследовательностью. Без ограничения общности можно считать, что $\left(y_{k}\right)$ сходится, скажем, к $y \in X$. Тогда $r=d(x, y)$. Поскольку $r>0$, для достаточно большого $k$ имеем $r_{k}>r / 2$ и $d\left(y, y_{k}\right)<r / 2$. Тогда в силу ультраметрического неравенства получаем, что

$$
r_{k} \leqslant \max \left(r, d\left(y, y_{k}\right)\right)=r
$$

и, аналогично,

$$
r \leqslant \max \left(r_{k}, d\left(y, y_{k}\right)\right)=r_{k}
$$

откуда $r_{k}=r$, что противоречит нашим предположениям. Лемма доказана.

ОПРЕДЕЛЕНИЕ 3.4. Для любого шара $B$ в $X$ обозначим через $\rho(B)$ его минимальный $d_{*}$-радиус.

Заметим, что $\rho(B)$ существует, поскольку все шары определяются как замкнутые шары, и является также $d_{*}$-диаметром шара $B$. 
Лемма 3.5. Если $\rho(B)>0$, то $\rho(B) \in \Lambda(x)$ для любого $x \in B$. Обратно, любое число в $\Lambda(x)$ равно $\rho(B)$ для некоторого шара $B$, содержащего $x$.

ДокАЗАТЕЛЬСтво. Положим $r=\rho(B)$, так что $B=B_{r}^{*}(x)$. Для любого $y \in$ $B$ имеем $d_{*}(x, y) \leqslant r$, и нам нужно показать, что $d_{*}(x, y)=r$ для некоторого $y$. Предположим, что $d_{*}(x, y)<r$ для всех $y \in B$. Тогда множество

$$
\left\{d_{*}(x, y): y \in B \backslash\{x\}\right\}
$$

является подмножеством из $(0, r) \cap \Lambda(x)$. В силу леммы 3.3 последнее множество обладает максимальным элементом, скажем, $r^{\prime}$. Тогда $B \subset B_{r^{\prime}}^{*}(x)$, что противоречит минимальности радиуса $r$. Обратно, если $r \in \Lambda(x)$, то шар $B=B_{r}(x)$ имеет радиус $\rho(B)=r$, поскольку существует $y \in X$ такое, что $d(x, y)=r$. Лемма доказана.

ОПРЕДЕЛЕНиЕ 3.6. Пусть $B, C$ - два шара в $X$ такие, что $C \subset B$. Будем говорить, что $C$ - последователь шара $B$ (а $B$ - предшественник шара $C$ ), если $C \neq B$ и для любого шара $A$ такого, что $C \subset A \subset B$, имеем $A=C$ или $A=B$. Другими словами, $B$ - минимальный шар, содержащий $C$ как собственное подмножество. Если $C$ - последователь шара $B$, будем писать $C \prec B$.

Обозначим через $\mathscr{K}$ семейство всех шаров $C$ в $X$ с положительными радиусами. Если $C=B_{r}^{*}(x)$ - шар из $\mathscr{K}$ с радиусом $r>0$, то для минимального радиуса $\rho(C)$ имеются две возможности:

1) $\rho(C)>0$,

2) $\rho(C)=0$ и центр шара $C$ является изолированной точкой пространства $X$.

Лемма 3.7. У любого шара $C \in \mathscr{K}$ такого, что $C \neq X$, есть единственный шар-предшественник $B$. Для любого шара $B$ с радиусом $\rho(B)>0$ число $\operatorname{deg}(B)$ его последователей удовлетворяет неравенствам $2 \leqslant \operatorname{deg}(B)<\infty$. Более того, все последователи шара $B$ не пересекаются и их обгединение равно $B$.

ДокАЗАтЕЛЬСтво. Зафиксируем некоторое $x \in C$. Из леммы 3.3 и определения $\mathscr{K}$ следует, что множество $(\rho(C), \infty) \cap \Lambda(x)$ имеет минимум, который мы обозначим через $r$. Тогда шар $B_{r}^{*}(x)$ является предшественником $C$. Единственность предшественника следует из определения.

Если $C_{1}$ и $C_{2}$ - два различных последователя шара $B$, то $C_{1}$ и $C_{2}$ не пересекаются. Действительно, если они пересекаются, то один из них содержит другой, скажем, $C_{1} \subset C_{2}$. По определению предшественника/последователя тогда должно выполняться равенство $C_{2}=C_{1}$ или $C_{2}=B$, откуда следует $C_{1}=C_{2}$.

Покажем, что для любого $x \in B$ найдется шар $C$ такой, что $x \in C \prec B$. Действительно, если множество $(0, \rho(B)) \cap \Lambda(x)$ пусто, то $C=B_{0}^{*}(x)=\{x\}-$ последователь шара $B$. Если множество $(0, \rho(B)) \cap \Lambda(x)$ непусто, то по лемме 3.3 у него есть максимум, скажем, $r$. Тогда $C=B_{r}^{*}(x)$ - последователь шара $B$. Отсюда следует, что множество всех последователей шара $B$ является покрытием шара $B$. 
Каждый последователь $C$ шара $B$ является открытым множеством (будучи также замкнутым шаром), так как $C$ совпадает с открытым шаром радиуса $\rho(B)$. Поскольку шар $B$ компактен, множество его последователей конечно, т. е. $\operatorname{deg}(B)<\infty$. Наконец, $\operatorname{deg}(B)$ не может быть равно 1 , так как тогда $B$ совпадал бы со своим единственным последователем. Отсюда следует, что $\operatorname{deg}(B) \geqslant 2$. Лемма доказана.

Для любого $C \in \mathscr{K}$ определим функцию $f_{C}$ на $X$ следующим образом. Если $C$ - собственное подмножество пространства $X$, то, обозначая через $B$ предшественника шара $C$, положим

$$
f_{C}=\frac{1}{\mu(C)} \mathbf{1}_{C}-\frac{1}{\mu(B)} \mathbf{1}_{B}
$$

(заметим, что всегда $\mu(C)>0$ ). Положим также $\lambda(C):=1 / \rho(B)$. Если $C=X$ (что может быть только в случае, когда $X$ компактно), то положим $f_{C} \equiv 1$ и $\lambda(C)=0$.

Теорема 3.8. Для любого $C \in \mathscr{K}$ функиия $f_{C}$ является собственной функиией оператора $\mathscr{L}$ с собственным значением $\lambda(C)$. Семейство $\left\{f_{C}: C \in \mathscr{K}\right\}$ полно (его линейная оболочка всюду плотна) в $L^{2}(X, \mu)$. Следовательно, оператор $\mathscr{L}$ обладает полной системой собственных функиий с компактными носителями.

ДокАЗАТЕЛЬство. Зафиксируем шар $C \in \mathscr{K}$ или радиус $r=\rho(C)$, и пусть $B$ - предшественник радиуса $r^{\prime}=\rho(B)$. Любой шар радиуса $s<r^{\prime}$ либо не пересекается с $C$, либо содержится в $C$, откуда следует, что $\mathbf{1}_{C}$ постоянна в любом таком шаре. Следовательно, для любого $s<r^{\prime}$ имеем $\mathrm{Q}_{s} \mathbf{1}_{C}=\mathbf{1}_{C}$ и, аналогично, $\mathrm{Q}_{s} \mathbf{1}_{B}=\mathbf{1}_{B}$, откуда

$$
\mathrm{Q}_{s} f_{C}=f_{C} .
$$

Если $s \geqslant r^{\prime}$, то любой шар радиуса $s$ либо содержит оба шара $C, B$, либо не пересекается с $B$. Поскольку средние двух функций $\frac{1}{\mu(C)} \mathbf{1}_{C}$ и $\frac{1}{\mu(B)} \mathbf{1}_{B}$ по любому шару, содержащему $C$ и $B$, равны, получаем, что в этом случае $\mathrm{Q}_{s} f_{C}=0$. Следовательно,

$$
\mathscr{L} f_{C}=-\int_{(0, \infty)} \frac{1}{s} \mathrm{Q}_{s} f_{C} d s=\frac{1}{r^{\prime}} f_{C}=\lambda(C) f_{C} .
$$

Это доказывает, что $f_{C}-$ собственная функция оператора $\mathscr{L}$ с собственным значением $\lambda(C)$. В случае компактного $X$ имеем $\mathrm{Q}_{s} f_{X}=f_{X}$ для всех $s>0$, откуда $\mathscr{L} f_{X}=0=\lambda(X)$.

Покажем, что система $\left\{f_{C}: C \in \mathscr{K}\right\}$ полна. Предположим, что некоторая функция $f \in L^{2}$ ортогональна всем функциям $f_{C}$, и докажем, что $f \equiv$ const. Для любого $r>0$ имеем

$$
\left(\mathrm{Q}_{r} f, f_{C}\right)_{L^{2}}=\left(f, \mathrm{Q}_{r} f_{C}\right)_{L^{2}}=\operatorname{const}\left(f, f_{C}\right)_{L^{2}}=0,
$$

где мы использовали то, что любая собственная функция оператора $\mathscr{L}$ является также собственной функцией оператора $\mathrm{Q}_{r}$ с собственным значением, 
которое мы обозначили через const. Отсюда следует, что $Q_{r} f$ также ортогональна всем $f_{C}$. Ниже мы докажем, что $\mathrm{Q}_{r} f=0$, из чего в силу $(2.2)$ будет следовать, что $f=0$.

Поскольку $\mathrm{Q}_{r} f$ постоянна в любом шаре радиуса $r$, то, переименовывая $\mathrm{Q}_{r} f$ обратно в $f$, мы можем с этого момента считать, что $f$ постоянна в любом шаре радиуса $r$. Зафиксируем некоторый шар $C \in \mathscr{K}$ и его предшественника $B$. Из (3.6) следует, что равенство $\left(f, f_{C}\right)_{L^{2}}=0$ равносильно равенству

$$
\frac{1}{\mu(C)} \int_{C} f d \mu=\frac{1}{\mu(B)} \int_{B} f d \mu
$$

т. е. среднее значение функции $f$ по шару сохраняется, когда мы переключаемся на его предшественника. Начиная с двух шаров $C_{1}$ и $C_{2}$ радиуса $r$, можно построить последовательности их предшественников, которые закончатся одним и тем же (достаточно большим) шаром. Отсюда следует, что средние значения функции $f$ в $C_{1}$ и $C_{2}$ одни и те же. Поскольку $f$ постоянна в $C_{1}$ и $C_{2}$, значения этих постоянных одинаковы. Следовательно, $f \equiv$ const на $X$. Если $\mu(X)=\infty$, то получим $f \equiv 0$. Если $\mu(X)<\infty$, то, используя ортогональность $f$ к $f_{X} \equiv 1$, снова получим, что $f \equiv 0$. Теорема доказана.

Для любого шара $B, \rho(B)>0$, определим подпространство $\mathscr{H}_{B}$ пространства $L^{2}$ следующим образом:

$$
\mathscr{H}_{B}=\operatorname{span}\left\{f_{C}: C \prec B\right\}
$$

По теореме 3.8 все ненулевые функции в $\mathscr{H}_{B}$ являются собственными функциями оператора $\mathscr{L}$ с собственным значением $1 / \rho(B)$.

Из леммы 3.7 следует, что функции $\left\{\mathbf{1}_{C}: C \prec B\right\}$ линейно независимы и

$$
\sum_{C \prec B} \mathbf{1}_{C}=\mathbf{1}_{B}
$$

Из этого следует, что

$$
\sum_{C \prec B} \mu(C) f_{C}=0
$$

и что это единственная зависимость между функциями $f_{C}$. Отсюда получаем, что

$$
\operatorname{dim} \mathscr{H}_{B}=\operatorname{deg}(B)-1 .
$$

Ясно, что пространства $\mathscr{H}_{B}$ и $\mathscr{H}_{B^{\prime}}$ ортогональны, как только шары $B, B^{\prime}$ не пересекаются.

Определим множество

$$
\Lambda:=\left\{d_{*}(x, y): x, y \in X, x \neq y\right\}=\bigcup_{x \in X} \Lambda(x) .
$$

Из теоремы 3.8 вытекает следующее. 
Следствие 3.9. Спектр $\operatorname{spec} \mathscr{L}$ лапласиана $\mathscr{L}$ чисто точечный $и$

$$
\operatorname{spec} \mathscr{L}=\overline{\left\{\frac{1}{r}: r \in \Lambda\right\}} \cup\{0\} .
$$

Пространство $L^{2}(X, \mu)$ разлагается в ортогональную сумму конечномерных собственных пространств следующим образом: если $\mu(X)=\infty$, то

$$
L^{2}(X, \mu)=\bigoplus_{\rho(B)>0} \mathscr{H}_{B}
$$

а если $\mu(X)<\infty$, mo

$$
L^{2}(X, \mu)=\{\text { const }\} \oplus \bigoplus_{\rho(B)>0} \mathscr{H}_{B} .
$$

ПримеР 3.10. Пусть $(X, d, \mu)$ такое же, как в примере 2.24 , т. е. $X=\mathbb{Q}_{p}$, $d(x, y)=\|x-y\|_{p}-p$-адическое расстояние, а $\mu$-мера Хаара. Для некоторого $\alpha>0$ положим

$$
\sigma(r)=\exp \left(-\left(\frac{p}{r}\right)^{\alpha}\right)
$$

так что в силу (2.39)

$$
d_{*}(x, y)=\left(\frac{\|x-y\|_{p}}{p}\right)^{\alpha} .
$$

Поскольку множество ненулевых значений расстояния $\|x-y\|_{p}$ есть $\left\{p^{k}\right\}_{k \in \mathbb{Z}}$, множество $\Lambda$ всех ненулевых значений расстояния $d_{*}(x, y)$ есть

$$
\Lambda=\left\{p^{\alpha k}: k \in \mathbb{Z}\right\}
$$

Отсюда следует, что

$$
\operatorname{spec} \mathscr{L}=\left\{p^{\alpha k}: k \in \mathbb{Z}\right\} \cup\{0\} .
$$

СлЕДСТвиЕ 3.11. Пусть $(X, d)$ - некомпактное ультраметрическое пространство с компактными шарами. Пусть $M \subset[0, \infty)$ - любое замкнутое множество (неограниченное, если X содержит хотя бы одну неизолированную точку), для которого 0 - точка сгущения. Тогда верно следующее.

(а) Существует ультраметрика $d^{\prime}$ на $X$ такая, что $d^{\prime}$-иары компактнь, $d^{\prime}$ порождает ту же топологию, что $u d$, и изотропный лапласиан $\mathscr{L}^{\prime}$, соответствующий тройке $\left(d^{\prime}, \mu, \sigma_{*}\right)$, имеет спектр $\operatorname{spec~} \mathscr{L}^{\prime}=M$.

(b) Предположим дополнительно, что существует разбиение $X$ на бесконечное множество d-шаров, каждый из которых содержит более одной точки. Тогда ультраметрику d' из пункта (а) можно выбрать так, чтобы семейства d-шаров и $d^{\prime}$-шаров совпадали.

ДоказАтельство. Множество

$$
D=\left\{x \in(0, \infty): x^{-1} \in M\right\} \cup\{0\}
$$

является замкнутым неограниченным подмножеством из $[0, \infty)$, содержащим 0 . Утверждение (а) равносильно существованию ультраметрики $d^{\prime}$ на $X$ с компактными шарами, которая порождает ту же топологию, что и $d$, и для которой замыкание множества значений $\left\{d^{\prime}(x, y)\right\}_{x, y \in X}$ этой метрики совпадает 
c D. Это метрическое свойство доказано А. Бендиковым и П. Крупским [8; $\S 2]$. Тогда изотропный лапласиан, связанный с тройкой $\left(d^{\prime}, \mu, \sigma_{*}\right)$, обладает требуемым свойством в силу следствия 3.9. Доказательство утверждения (b) следует таким же образом из [8; §2]. Следствие доказано.

3.3. Форма Дирихле и плотность скачков. Построим форму Дирихле $\left(\mathscr{E}, \operatorname{dom}_{\mathscr{E}}\right)$, связанную с изотропной полугруппой $\left\{P^{t}\right\}$. Хорошо известно, что если $P^{t} 1=1$, как в нашем случае, то

$$
\mathscr{E}(f, f)=\lim _{t \rightarrow 0} \frac{1}{2 t} \int_{X} \int_{X} p(t, x, y)(f(x)-f(y))^{2} d \mu(x) d \mu(y)
$$

и

$$
\operatorname{dom}_{\mathscr{E}}=\left\{f \in L^{2}: \mathscr{E}(f, f)<\infty\right\}
$$

(см. [27]). Используя тождество (2.18), получаем, что

$$
\frac{p(t, x, y)}{t} \nearrow \int_{0}^{1 / d_{*}(x, y)} N(x, \tau) d \tau \quad \text { при } t \searrow 0 .
$$

Полагая

$$
J(x, y):=\int_{0}^{1 / d_{*}(x, y)} N(x, \tau) d \tau=\int_{d_{*}(x, y)}^{\infty} \frac{1}{V(x, s)} \frac{d s}{s^{2}},
$$

по теореме о монотонной сходимости получаем, что для всех $f \in L^{2}$

$$
\mathscr{E}(f, f)=\frac{1}{2} \int_{X} \int_{X}(f(x)-f(y))^{2} J(x, y) d \mu(x) d \mu(y) .
$$

Заметим, что $0<J(x, y)=J(y, x)<\infty$ для всех $x \neq y$, тогда как $J(x, x)=\infty$.

Из тождества поляризации тогда следует, что для всех $f, g \in \operatorname{dom}_{\mathscr{E}}$

$$
\mathscr{E}(f, g)=\frac{1}{2} \int_{X} \int_{X}(f(x)-f(y))(g(x)-g(y)) J(x, y) d \mu(x) d \mu(y) .
$$

Функция $J$ называется плотностью скачков формы Дирихле $\mathscr{E}$. Здесь мы покажем, что ее можно использовать также для описания генератора $\mathscr{L}$ полугруппы $\left\{P^{t}\right\}$. Напомним, что в силу теории форм Дирихле генератор $\mathscr{L}$ имеет следующее равносильное определение: это самосопряженный оператор в $L^{2}$ с $\operatorname{dom}_{\mathscr{L}} \subset \operatorname{dom}_{\mathscr{E}}$ такой, что

$$
(\mathscr{L} f, g)=\mathscr{E}(f, g)
$$

для всех $f \in \operatorname{dom}_{\mathscr{L}}$ и $g \in \operatorname{dom}_{\mathscr{E}}$.

Обозначим через $\mathscr{V}_{r}$ образ оператора $\mathrm{Q}_{r}$ (определенного относительно $d_{*}$ ), т. е. пространство всех $L^{2}$-функций, постоянных на каждом шаре радиуса $r$. Положим также

$$
\mathscr{V}:=\bigcup_{r>0} \mathscr{V}_{r}
$$

и заметим, что $\mathscr{V}$ - линейное подпространство в $L^{2}$. Заметим также, что пространство $\mathscr{V}_{c}$ всех локально постоянных функций с компактным носителем содержится в $\mathscr{V}$. 
ТЕОрема 3.12. Пространство $\mathscr{V}$ всюду плотно в $L^{2}$, является подмножеством в $\operatorname{dom}_{\mathscr{L}}$ и для любого $f \in \mathscr{V}$

$$
\mathscr{L} f(x)=\int_{X}(f(x)-f(y)) J(x, y) d \mu(y) .
$$

ДоказАтельство. Тот факт, что $\mathscr{V}$ всюду плотно в $L^{2}$, следует из (2.2). На самом деле, $\mathscr{V}_{c}$ также всюду плотно в $L^{2}$, что следует из того, что все собственные функции оператора $\mathscr{L}$ лежат в $\mathscr{V}_{c}$.

В силу (2.6) и (3.4) имеем $\mathrm{Q}_{r}=\mathbf{1}_{[0,1 / r)}(\mathscr{L})$. Значит, $\mathscr{L} \mathrm{Q}_{r}$ - ограниченный оператор, откуда следует, что $\operatorname{dom}_{\mathscr{L}} \supset \mathscr{V}_{r}$ и потому $\operatorname{dom}_{\mathscr{L}} \supset \mathscr{V}$.

Зафиксируем функцию $f \in \mathscr{V}_{r}$ с $r>0$ и положим

$$
u(x)=\int_{X}|f(x)-f(y)| J(x, y) d \mu(y) .
$$

Покажем, что $u \in L^{2}$. Заметим, что $f(x)=f(y)$, как только выполнено неравенство $d_{*}(x, y) \leqslant r$. Отсюда следует, что можно ограничить интегрирование на область $\left\{d_{*}(x, y)>r\right\}$. В силу неравенства Коши-Шварца имеем

$$
u^{2}(x) \leqslant\left(\int_{X}|f(x)-f(y)|^{2} J(x, y) d \mu(y)\right)\left(\int_{\left\{y: d_{*}(x, y)>r\right\}} J(x, y) d \mu(y)\right) .
$$

Покажем, что

$$
\int_{\left\{y: d_{*}(x, y)>r\right\}} J(x, y) d \mu(y) \leqslant \frac{1}{r} .
$$

Действительно, в силу (3.11) и теоремы Фубини последний интеграл равен

$$
\begin{aligned}
\int_{\left\{y: d_{*}(x, y)>r\right\}} & \int_{\left\{s: s \geqslant d_{*}(x, y)\right\}}^{\infty} \frac{1}{V(x, s)} \frac{d s}{s^{2}} d \mu(y) \\
& =\int_{r}^{\infty} \frac{d s}{s^{2} V(x, s)} \int_{\left\{y: r<d_{*}(x, y) \leqslant s\right\}} d \mu(y) \\
& =\int_{r}^{\infty} \frac{V(x, s)-V(x, r)}{s^{2} V(x, s)} d s \\
& \leqslant \int_{r}^{\infty} \frac{d s}{s^{2}}=\frac{1}{r} .
\end{aligned}
$$

Из (3.14) следует, что

$$
\int_{X} u^{2} d \mu \leqslant \frac{1}{r} \mathscr{E}(f, f) .
$$

Поскольку $f \in \operatorname{dom}_{\mathscr{L}} \subset \operatorname{dom}_{\mathscr{E}}$, получаем, что $u \in L^{2}$. В частности, $u(x)<\infty$ для почти всех $x \in X$. Следовательно, для почти всех $x \in X$ функция

$$
y \mapsto(f(x)-f(y)) J(x, y)
$$

лежит в $L^{1}$ и ее интеграл

$$
v(x)=\int_{X}(f(x)-f(y)) J(x, y) d \mu(y)
$$


является $L^{2}$-функцией. Нам нужно проверить, что $\mathscr{L} f=v$. Для этого достаточно проверить, что для любого $g \in \operatorname{dom}_{\mathscr{E}}$

$$
(v, g)_{L^{2}}=\mathscr{E}(f, g) .
$$

Действительно, используя теорему Фубини, получаем

$$
\begin{aligned}
(v, g)_{L^{2}} & =\int_{X} \int_{X}(f(x)-f(y)) g(x) J(x, y) d \mu(y) d \mu(x) \\
& =\int_{X} \int_{X}(f(y)-f(x)) g(y) J(y, x) d \mu(x) d \mu(y) \\
& =\frac{1}{2} \int_{X} \int_{X}(f(x)-f(y))(g(x)-g(y)) J(x, y) d \mu(x) d \mu(y) \\
& =\mathscr{E}(f, g)
\end{aligned}
$$

что и требовалось доказать. Теорема доказана.

3.4. $L^{p}$-спектр лапласиана. Известно, что любую непрерывную симметричную марковскую полугруппу можно продолжить на все пространства $L^{p}$, $1 \leqslant p<\infty$, как непрерывную сжимающую полугруппу. В частности, это верно для полугруппы $\left\{P^{t}\right\}$. Мы используем те же обозначения для продолженной полугруппы и обозначаем через $\mathscr{L}_{p}$ ее инфинитезимальный генератор, а через $\operatorname{dom}_{\mathscr{L}_{p}}$ ее область определения в $L^{p}$.

Теорема 3.13. Для всех $1 \leqslant p<\infty$ имеем

$$
\operatorname{spec} \mathscr{L}_{p}=\operatorname{spec} \mathscr{L}_{2} \text {. }
$$

ДокАЗАТЕЛьСтво. Поскольку по теореме 3.8 все собственные функции оператора $\mathscr{L}_{2}$ имеют компактные носители, они принадлежат также $L^{p}$, откуда следует, что

$$
\operatorname{spec} \mathscr{L}_{2} \subset \operatorname{spec} \mathscr{L}_{p}
$$

Чтобы доказать обратное включение, выберем $\lambda_{0} \notin \operatorname{spec} \mathscr{L}_{2}$ и покажем, что $\lambda_{0} \notin \operatorname{spec} \mathscr{L}_{p}$. Для этого достаточно показать, что резольвента

$$
R:=\left(\mathscr{L}_{2}-\lambda_{0} \mathrm{id}\right)^{-1},
$$

будучи ограниченным оператором в $L^{2}$, продолжается до ограниченного оператора в $L^{p}$. Последнее сводится к тому, чтобы показать, что для любых функций $f \in L^{2} \cap L^{p}$ и $g \in L^{2} \cap L^{q}$, где $q=p /(p-1)$ - гёльдеров сопряженный показателя $p$, выполняется неравенство

$$
\left|(R f, g)_{L^{2}}\right| \leqslant C\|f\|_{L^{p}}\|g\|_{L^{q}}
$$

с постоянной $C$, не зависящей от $f, g$.

Ограничимся случаем $\lambda_{0}>0$ (случай $\lambda_{0}<0$ проще). Выберем $a, b>0$ такие, что $a<\lambda_{0}<b$ и $[a, b]$ не пересекается с $\operatorname{spec} \mathscr{L}_{2}$. Используя спектральное разложение (3.4), получим

$$
R=\int_{\operatorname{spec} \mathscr{L}_{2}} \frac{d E_{\lambda}}{\lambda-\lambda_{0}}=\int_{[0, a)} \frac{d E_{\lambda}}{\lambda-\lambda_{0}}+\int_{[b, \infty)} \frac{d E_{\lambda}}{\lambda-\lambda_{0}},
$$


откуда

$$
(R f, g)=\int_{[0, a)} \frac{d\left(E_{\lambda} f, g\right)}{\lambda-\lambda_{0}}+\int_{[b, \infty)} \frac{d\left(E_{\lambda} f, g\right)}{\lambda-\lambda_{0}} .
$$

Интегрирование по частям дает

$$
\begin{aligned}
(R f, g)= & \frac{\left(E_{a} f, g\right)}{a-\lambda_{0}}+\int_{[0, a)} \frac{\left(E_{\lambda} f, g\right)}{\left(\lambda-\lambda_{0}\right)^{2}} d \lambda \\
& \quad-\frac{\left(E_{b} f, g\right)}{b-\lambda_{0}}+\int_{[b, \infty)} \frac{\left(E_{\lambda} f, g\right)}{\left(\lambda-\lambda_{0}\right)^{2}} d \lambda .
\end{aligned}
$$

Поскольку $E_{\lambda}=Q_{1 / \lambda}$ - марковский оператор, он стандартным образом продолжается до ограниченного оператора в $L^{p}$ с оценкой нормы 1 , так что

$$
\left|\left(E_{\lambda} f, g\right)\right| \leqslant\|f\|_{L^{p}}\|g\|_{L^{q}}
$$

Следовательно,

$$
|(R f, g)| \leqslant\|f\|_{L^{p}}\|g\|_{L^{q}}\left(\frac{1}{\lambda_{0}-a}+\frac{1}{b-\lambda_{0}}+\int_{[0, a) \cup[b, \infty)} \frac{d \lambda}{\left(\lambda-\lambda_{0}\right)^{2}}\right),
$$

что завершает доказательство, поскольку величина в больших скобках конечна. Теорема доказана.

Последняя теорема этого раздела относится к свойству Лиувилля. Заметим, что полугруппа $\left\{P^{t}\right\}$, определенная равенством (1.6), действует на пространстве $\mathscr{B}_{b}$ ограниченных борелевских функций как сжимающая полугруппа, но она не непрерывна, если только $X$ не дискретно. Определим сходимость последовательности в $\mathscr{B}_{b}$ как ограниченную поточечную сходимость, т. е. последовательность $\left\{f_{k}\right\} \subset \mathscr{B}_{b}$ сходится в $\mathscr{B}_{b}$ к функции $f$, если вся последовательность $\left\{f_{k}\right\}$ равномерно ограничена и $f_{k}(x) \rightarrow f(x)$ при $k \rightarrow \infty$ для всех $x \in X$. Определим слабый инфинитезимальный генератор $\mathscr{L}_{\infty}$ полугруппы $\left\{P^{t}\right\}$ в $\mathscr{B}_{b}$ следующим образом: область определения $\operatorname{dom}_{\mathscr{L}_{\infty}}$ состоит из функций $f \in \mathscr{B}_{b}$ таких, что предел

$$
\mathscr{L}_{\infty} f:=\lim _{t \rightarrow 0} \frac{f-P^{t} f}{t}
$$

существует в смысле сходимости в $\mathscr{B}_{b}$. Это дает $\mathscr{L}_{\infty} f \in \mathscr{B}_{b}$ для любого $f \in$ $\operatorname{dom}_{\mathscr{L}_{\infty}}$.

ТЕОРема 3.14 (сильное свойство Лиувилля). Любая борелевская функиия $f: X \rightarrow[0, \infty)$, удовлетворяющая $P f=f$, долюна быть постоянной.

Следовательно, 0 - собственное значение оператора $\mathscr{L}_{\infty}$ кратности 1.

Доказательство. Поскольку $P$ и $\mathrm{Q}_{r}$ перестановочны, из $f=P f$ и

$$
P f=\int_{0}^{\infty} \mathrm{Q}_{s} f d \sigma_{*}(s)
$$

получаем, что для всех $r \geqslant 0$

$$
\mathrm{Q}_{r} f=P \mathrm{Q}_{r} f=\int_{[0, \infty)} \mathrm{Q}_{s} \mathrm{Q}_{r} f d \sigma_{*}(s) .
$$


Замечая, что

$$
\mathrm{Q}_{s} \mathrm{Q}_{r}=\mathrm{Q}_{\max (r, s)}
$$

получаем

$$
\mathrm{Q}_{r} f=\int_{[0, r)} \mathrm{Q}_{r} f d \sigma_{*}(s)+\int_{[r, \infty)} \mathrm{Q}_{s} f d \sigma_{*}(s) .
$$

Первый интеграл здесь равен $\sigma_{*}(r) \mathrm{Q}_{r} f$, откуда следует равенство

$$
\left(1-\sigma_{*}(r)\right) \mathrm{Q}_{r} f=\int_{[r, \infty)} \mathrm{Q}_{s} f d \sigma_{*}(s) .
$$

Зафиксируем некоторый $x \in X$. В силу леммы 3.3 множество $\Lambda(x)$ всех значений $d_{*}(x, y)$ при $y \neq x$ не имеет точек сгущения в $(0,+\infty)$. Выберем $r_{0}$ следующим образом: если $\Lambda(x)$ не имеет точки сгущения в 0 , то $r_{0}=0$, а если $\Lambda(x)$ имеет точку сгущения в 0 , то $r_{0}$ - любое значение из $\Lambda(x)$. В обоих случаях множество $\Lambda(x) \cap(r, \infty)$ состоит из (конечной или бесконечной) последовательности $r_{1}<r_{2}<\cdots$, которая сходится к $\infty$ в случае, когда она бесконечна. Применяя (3.16) к $r=r_{k}$ и $r=r_{k+1}$, где $k \geqslant 0$, получаем

$$
\begin{aligned}
\left(1-\sigma_{*}\left(r_{k}\right)\right) \mathrm{Q}_{r_{k}} f(x)-\left(1-\sigma_{*}\left(r_{k+1}\right)\right) \mathrm{Q}_{r_{k+1}} f(x) & =\int_{\left[r_{k}, r_{k+1}\right)} \mathrm{Q}_{s} f(x) d \sigma_{*}(s) \\
& =\mathrm{Q}_{r_{k}} f(x)\left(\sigma_{*}\left(r_{k+1}\right)-\sigma_{*}\left(r_{k}\right)\right),
\end{aligned}
$$

откуда следует, что

$$
\left(1-\sigma_{*}\left(r_{k+1}\right)\right) \mathrm{Q}_{r_{k}} f(x)=\left(1-\sigma_{*}\left(r_{k+1}\right)\right) \mathrm{Q}_{r_{k+1}} f(x)
$$

и потому

$$
\mathrm{Q}_{r_{k}} f(x)=\mathrm{Q}_{r_{k+1}} f(x)
$$

Следовательно,

$$
\mathrm{Q}_{r_{k}} f(x)=\mathrm{Q}_{r_{0}} f(x) \quad \text { для всех } k \geqslant 1 .
$$

Поскольку $r_{0}$ можно выбрать произвольно близким к 0 , получаем, что $\mathrm{Q}_{r} f(x)$ не зависит от $r$. Для любых двух точек $x, y \in X$ имеем $\mathrm{Q}_{r} f(x)=\mathrm{Q}_{r} f(y)$ при $r \geqslant d_{*}(x, y)$. Поэтому функция $\mathrm{Q}_{r} f(x)$ постоянна и по $r$, и по $x$. Из $(3.15)$ следует, что $f=P f$ также постоянна.

Чтобы доказать второе утверждение теоремы, заметим, что 0 является собственным значением оператора $\mathscr{L}_{\infty}$, поскольку $\mathscr{L}_{\infty} 1=0$. Предположим, что $\mathscr{L}_{\infty} f=0$, и докажем, что $f \equiv$ const, откуда будет следовать, что кратность собственного значения 0 равна 1. По предположению имеем $f \in \mathscr{B}_{b}$ и

$$
\frac{f-P^{t} f}{t} \stackrel{\mathscr{B}_{b}}{\longrightarrow} 0 \quad \text { при } t \rightarrow 0 .
$$

Поскольку семейство $\left\{\frac{f-P^{t} f}{t}\right\}_{t>0}$ равномерно ограничено, по теореме о мажорируемой сходимости получаем, что для любого $r \geqslant 0$

$$
\mathrm{Q}_{r}\left(\frac{f-P^{t} f}{t}\right) \stackrel{\mathscr{B}_{b}}{\longrightarrow} 0 \quad \text { при } t \rightarrow 0
$$


что в свою очередь влечет, что для всех $s \geqslant 0$

$$
\frac{P^{s} f-P^{s+t} f}{t}=P^{s}\left(\frac{f-P^{t} f}{t}\right) \stackrel{\mathscr{B}_{b}}{\longrightarrow} 0 \quad \text { при } t \rightarrow 0 .
$$

Следовательно, для любого $x \in X$ функция $s \mapsto P^{s} f(x)$ имеет производную, равную 0 , и потому постоянна. Значит, $f=P f$, и в силу первого утверждения теоремы мы заключаем, что $f=$ const. Теорема доказана.

\section{4. Моменты марковского процесса}

Пусть $\left\{\mathscr{X}_{t}\right\}-$ марковский процесс, связанный с полугруппой $\left\{P^{t}\right\}$. Для любого $\gamma>0$ момент процесса порядка $\gamma$ определяется формулой

$$
M_{\gamma}(x, t)=\mathrm{E}_{x}\left(d_{*}\left(x, \mathscr{X}_{t}\right)^{\gamma}\right),
$$

где $\mathrm{E}_{x}$ - математическое ожидание относительно вероятностной меры на пространстве траекторий процесса $\left\{\mathscr{X}_{t}\right\}$ с $\mathscr{X}_{0}=x$. В терминах ядра теплопроводности $p(t, x, y)$ момент задается равенством

$$
M_{\gamma}(x, t)=\int_{X} d_{*}(x, y)^{\gamma} p(t, x, y) d \mu(y) .
$$

Целью этого раздела является оценка $M_{\gamma}(x, t)$ как функции от $t$ и $\gamma$.

Начнем с двух лемм. Мы используем функцию объема (2.48), т. е.

$$
V(x, r)=\mu\left(B_{r}^{*}(x)\right),
$$

и ее усредненные моменты порядка $\gamma$, т. е.

$$
R_{\gamma}(x, \tau)=\frac{1}{V(x, \tau)} \int_{(0, \tau]} r^{\gamma} d V(x, r)
$$

Лемма 4.1. Для всех $x \in X, t>0 u \gamma>0$

$$
M_{\gamma}(x, t)=t \int_{0}^{\infty} R_{\gamma}\left(x, \frac{1}{\tau}\right) e^{-\tau t} d \tau=\int_{0}^{\infty} R_{\gamma}\left(x, \frac{t}{s}\right) e^{-s} d s .
$$

ДокАЗАТЕЛЬство. Используя равенства (4.1) и (2.24), а также определение 2.8 спектральной функции в терминах функции объема, получаем

$$
\begin{aligned}
M_{\gamma}(x, t) & =\int_{X} d_{*}(x, y)^{\gamma} p(t, x, y) d \mu(y) \\
& =\int_{(0, \infty)} r^{\gamma}\left(t \int_{0}^{1 / r} N(x, \tau) e^{-\tau t} d \tau\right) d V(x, r) \\
& =\int_{0}^{\infty}\left(\int_{(0,1 / \tau)} \frac{r^{\gamma}}{V(x, 1 / \tau)} d V(x, r)\right) t e^{-\tau t} d \tau=\int_{0}^{\infty} R_{\gamma}\left(x, \frac{1}{\tau}\right) t e^{-\tau t} d \tau .
\end{aligned}
$$

В третьем тождестве мы использовали теорему Фубини. Лемма доказана. 
Функция объема $r \mapsto V(x, r)$ не убывает и принимает значения от 0 до $\mu(X)$. В компактном случае имеем $V(x, r)=\mu(X)$ для всех $r \geqslant r_{\max }^{*}=r_{\max }^{*}(x)$, где $r_{\max }^{*}(x)$ - наибольшее значение в $\Lambda(x)$ (см. (3.5)). Если точка $x$ изолирована, то $V(x, r)=\mu\{x\}$ для всех $0 \leqslant r<r_{0}^{*}=r_{0}^{*}(x)$, где $r_{0}^{*}(x)$ - наименьшее положительное значение в $\Lambda(x)$.

ЛЕмма 4.2. Для любых заданных $x \in X u \gamma>0$ выполняются следующие свойства.

(а) Функиия $\tau \mapsto R_{\gamma}(x, \tau)$ неубъвающая.

Если $X$ компактно, mо $R_{\gamma}(x, \tau)=R_{\gamma}\left(x, r_{\max }^{*}(x)\right)$ для всех $\tau \geqslant r_{\max }^{*}(x)$. Если $X$ дискретно и бесконечно, то $R_{\gamma}(x, \tau)=R_{\gamma}\left(x, r_{0}^{*}(x)\right)$ для всех $0<\tau \leqslant r_{0}^{*}(x)$.

(b) Для всех $\tau>0$ выполняется неравенство

$$
R_{\gamma}(x, \tau) \leqslant \tau^{\gamma}
$$

и если функиия обгема $r \mapsto V(x, r)$ удовлетворяет обратному свойству удвоения, то существует постоянная с >0 такая, что

$$
R_{\gamma}(x, \tau) \geqslant c \tau^{\gamma}
$$

для всех $\tau>0$. В недискретном компактном случае, если функиия объема всего лишь удовлетворяет обратному свойству удвоения в нуле, то (4.2) выполняется для всех $0<\tau<r_{\max }^{*}(x)$. В дискретном бесконечном случае, если функция обгема всего лишь удовлетворяет обратному свойству удвоения на бесконечности, то (4.2) выполняется для всех $\tau>r_{0}^{*}(x)$.

ДокАзАТЕЛьство. Чтобы доказать первую часть пункта (а), проинтегрируем по частям:

$$
\begin{aligned}
R_{\gamma}(x, \tau) & =\frac{1}{V(x, \tau)}\left(\tau^{\gamma} V(x, \tau)-\int_{(0, \tau]} V(x, s) d s^{\gamma}\right) \\
& =\int_{(0, \tau]}\left(1-\frac{V(x, s)}{V(x, \tau)}\right) d s^{\gamma}
\end{aligned}
$$

откуда следует, что функция $\tau \mapsto R_{\gamma}(x, \tau)$ неубывающая.

Оставшаяся часть пункта (а) несложна.

Что касается пункта (b), общая верхняя оценка на $R_{\gamma}(x, \tau)$ очевидна. Если функция объема удовлетворяет обратному свойству удвоения, то в соответствующей области значений имеем

$$
\begin{aligned}
R_{\gamma}(x, \tau) & \geqslant \frac{1}{V(x, \tau)}(\delta \tau)^{\gamma}(V(x, \tau)-V(x, \delta \tau)) \\
& =(\delta \tau)^{\gamma}\left(1-\frac{V(x, \delta \tau)}{V(x, \tau)}\right) \geqslant \delta^{\gamma}(1-\kappa) \tau^{\gamma}=c \tau^{\gamma}
\end{aligned}
$$

для подходящих постоянных $0<\kappa, c<1$. Лемма доказана. 
Теперь, чтобы оценить функцию момента $t \mapsto M_{\gamma}(x, t)$, нам нужно оценить интеграл типа Лапласа, заданный формулой из леммы 4.1. Мы займемся такими оценками в двух технических предложениях 4.6 и 4.7 в конце этого раздела. Перед этим, в следующих трех теоремах, мы сформулируем результаты, касающиеся функции момента.

Теорема 4.3. Предположим, что $(X, d)$ не компактно и не имеет изолированных точек. Тогда выполняются следующие свойства.

(1) Для всех $x \in X, t>0 u 0<\gamma<1$

$$
M_{\gamma}(x, t) \leqslant \frac{t^{\gamma}}{1-\gamma}
$$

(2) Если для некоторого $x \in X$ функиия объема удовлетворяет обратному свойству удвоения, то при любом $0<\gamma<1$

$$
M_{\gamma}(x, t) \geqslant \frac{c}{1-\gamma} t^{\gamma}
$$

для всех $x, t>0$ и некоторого $c>0$. Более того,

$$
M_{\gamma}(z, t)=\infty
$$

для всех $z, t>0 u \gamma \geqslant 1$.

Теорема 4.4. Предположим, что $(X, d)$ дискретно и бесконечно. Тогда выполняются следующие свойства.

(а) Для всех $x, t>0 u 0<\gamma<1$

$$
M_{\gamma}(x, t) \leqslant \frac{C}{1-\gamma} \min \left\{t, t^{\gamma}\right\}
$$

с некоторым $C>0$.

(b) Если для некоторого (равносильно, для всех) $x \in X$ функиия обгема удовлетворяет обратному свойству удвоения на бесконечности, то при любом $0<\gamma<1$

$$
M_{\gamma}(z, t) \geqslant \frac{c}{1-\gamma} \min \left\{t, t^{\gamma}\right\}
$$

для всех $z, t>0$ и для некоторого с $>0$. Более того,

$$
M_{\gamma}(z, t)=\infty
$$

для всех $z, t>0$ и всех $\gamma \geqslant 1$.

Предположим теперь, что $(X, d)$ компактно, и пусть $D$ - его $d_{*}$-диаметр. В силу лемм 4.1 и 4.2 для всех $x \in X, \gamma>0$ и $t>0$ имеем

$$
M_{\gamma}(x, t) \leqslant R_{\gamma}(x, D) \leqslant D^{\gamma}
$$

откуда мы получаем информацию о поведении функции момента $t \mapsto M_{\gamma}(x, t)$ в нуле. 
Теорема 4.5. Предположим, что $(X, d)$ не дискретно и компактно. Тогда выполняются следующие свойства.

(1) Существует такая постоянная $C>0$, что для всех $x$ и всех $0<t \leqslant 1$ выполняется неравенство

$$
M_{\gamma}(x, t) \leqslant C \begin{cases}t, & \text { если } \gamma>1 \\ t\left(\log \frac{1}{t}+1\right), & \text { если } \gamma=1 \\ t^{\gamma}, & \text { если } \gamma<1\end{cases}
$$

(2) Если для некоторого $x \in X$ функиия объема удовлетворяет обратному свойству удвоения в нуле, то существует такая постоянная $c>0$, что для всех z и всех $0<t \leqslant 1$ выполняется неравенство

$$
M_{\gamma}(z, t) \geqslant c \begin{cases}t, & \text { если } \gamma>1, \\ t\left(\log \frac{1}{t}+1\right), & \text { если } \gamma=1, \\ t^{\gamma}, & \text { если } \gamma<1 .\end{cases}
$$

Теперь мы приведем технические подробности относительно оценок типа Лапласа, из которых следуют теоремы 4.3, 4.4 и 4.5. В следующих двух предложениях $M$ и $R$ - две неотрицательные неубывающие функции, связанные интегралом типа Лапласа

$$
M(t)=\int_{0}^{\infty} R\left(\frac{t}{\tau}\right) e^{-\tau} d \tau .
$$

ПРЕДЛОЖЕНИЕ 4.6. Пусть задано $\gamma>0$.

(1) Предположим, что

$$
A s^{\gamma} \geqslant R(s) \quad\left(\text { соответственно } R(s) \geqslant B s^{\gamma}\right)
$$

для некоторого $A>0$ (соответственно $B>0)$ и всех $s>0$. Тогда для всех $0<\gamma<1$ и всех $t>0$ выполняется неравенство

$$
\frac{A t^{\gamma}}{1-\gamma} \geqslant M(t) \quad\left(\text { соответственно } M(t) \geqslant \frac{B t^{\gamma}}{(1-\gamma) e}\right) .
$$

(2) Предположим, что имеется $t_{0}>0$ такое, что $R(s)=0$ для всех $0<$ $s<t_{0}$. Предположим также, что первое (соответственно второе) неравенство в (4.3) выполняется для всех $s>t_{0}$. Тогда

$$
\begin{aligned}
M(t) & \leqslant \frac{c}{1-\gamma} \min \left\{\frac{t}{t_{0}},\left(\frac{t}{t_{0}}\right)^{\gamma}\right\} \\
(\text { соответственно } M(t) & \left.\geqslant \frac{c^{\prime}}{1-\gamma} \min \left\{\frac{t}{t_{0}},\left(\frac{t}{t_{0}}\right)^{\gamma}\right\}\right)
\end{aligned}
$$

для всех $0<\gamma<1$, всех $t>0$ и некоторых постоянных $c, c^{\prime}>0$.

(3) Из предположения $\gamma \geqslant 1$ и нижней оценки $R(s) \geqslant B s^{\gamma}$ следует, что $M(t)=\infty$ для всех $t>0$. 
ДокАЗАТЕльство. Известно, что для $0<\gamma<1$ гамма-функция удовлетворяет неравенствам

$$
\frac{1}{(1-\gamma) e}<\Gamma(1-\gamma)<\frac{1}{1-\gamma}
$$

откуда в силу монотонности интеграла типа Лапласа вытекает первое утверждение.

Чтобы доказать второе утверждение, запишем

$$
M(t)=\int_{\left\{t / s \geqslant t_{0}\right\}} R\left(\frac{t}{s}\right) e^{-s} d s .
$$

Сначала предположим, что $R(s) \leqslant A s^{\gamma}$ для всех $0<s<\infty$. Тогда получим

$$
\begin{aligned}
M(t) & \leqslant A \int_{\left\{t / s \geqslant t_{0}\right\}}\left(\frac{t}{s}\right)^{\gamma} e^{-s} d s=A t^{\gamma} \int_{\left\{s \leqslant t / t_{0}\right\}} s^{-\gamma} e^{-s} d s \\
& \leqslant A t^{\gamma} \int_{0}^{t / t_{0}} s^{-\gamma} d s=\left(\frac{t}{t_{0}}\right) \frac{A t_{0}^{-\gamma}}{1-\gamma}
\end{aligned}
$$

и

$$
M(t) \leqslant A t^{\gamma} \int_{0}^{\infty} s^{-\gamma} e^{-s} d s \leqslant \frac{A t^{\gamma}}{1-\gamma}=\left(\frac{t}{t_{0}}\right)^{\gamma} \frac{A t_{0}^{\gamma}}{1-\gamma} .
$$

Следовательно,

$$
M(t) \leqslant \frac{A \max \left\{t_{0}, t_{0}^{-1}\right\}}{1-\gamma} \min \left\{\frac{t}{t_{0}},\left(\frac{t}{t_{0}}\right)^{\gamma}\right\}
$$

Теперь предположим, что $R(s) \geqslant B s^{\gamma}$ для всех $s \geqslant t_{0}$. Тогда при $t / t_{0} \geqslant 1$ имеем

$$
M(t) \geqslant B t^{\gamma} \int_{0}^{t / t_{0}} s^{-\gamma} e^{-s} d s \geqslant \frac{B t^{\gamma}}{e} \int_{0}^{1} s^{-\gamma} d s=\frac{B t^{\gamma}}{(1-\gamma) e}=\frac{B t_{0}^{\gamma}}{(1-\gamma) e}\left(\frac{t}{t_{0}}\right)^{\gamma} .
$$

Когда $t / t_{0} \leqslant 1$, получаем

$$
\begin{aligned}
M(t) & \geqslant B t^{\gamma} \int_{0}^{t / t_{0}} s^{-\gamma} e^{-s} d s \geqslant \frac{B t^{\gamma}}{e} \int_{0}^{t / t_{0}} s^{-\gamma} d s \\
& =\frac{B t^{\gamma}}{(1-\gamma) e}\left(\frac{t}{t_{0}}\right)^{1-\gamma}=\frac{B t_{0}^{\gamma}}{(1-\gamma) e}\left(\frac{t}{t_{0}}\right) .
\end{aligned}
$$

Следовательно,

$$
M(t) \geqslant \frac{B t_{0}^{\gamma}}{(1-\gamma) e} \min \left\{\frac{t}{t_{0}},\left(\frac{t}{t_{0}}\right)^{\gamma}\right\} \geqslant \frac{B \min \left\{t_{0}, 1\right\}}{(1-\gamma) e} \min \left\{\frac{t}{t_{0}},\left(\frac{t}{t_{0}}\right)^{\gamma}\right\} .
$$

Это доказывает второе утверждение. 
Для доказательства третьего утверждения заметим, что если $R(s) \geqslant B s^{\gamma}$ для всех $s \geqslant t_{0}$ и $\gamma \geqslant 1$, то

$$
M(t) \geqslant B t^{\gamma} \int_{0}^{t / t_{0}} s^{-\gamma} e^{-s} d s=\infty
$$

для всех $t>0$. Предложение доказано.

ПреДЛОЖЕНИЕ 4.7. Предположим, что имеется $t_{0}>0$ maкое, что $R(s)=$ $R\left(t_{0}\right)$ для всех $s \geqslant t_{0}$. Предположим также, что первое (соответственно второе) неравенство в (4.3) выполняется для всех $0<s \leqslant t_{0}$. Тогда

$$
\begin{aligned}
M(t) \leqslant \begin{cases}c_{1} \frac{t}{t_{0}}, & \text { если } \gamma>1, \\
c_{2} t\left(\log \frac{t_{0}}{t}+1\right), & \text { если } \gamma=1, \\
c_{3}\left(\frac{t}{t_{0}}\right)^{\gamma}, & \text { если } \gamma<1,\end{cases} \\
\text { соответственно } M(t) \geqslant \begin{cases}c_{1}^{\prime} \frac{t}{t_{0}}, & \text { если } \gamma>1, \\
c_{2}^{\prime} t\left(\log \frac{t_{0}}{t}+1\right), & \text { если } \gamma=1, \\
c_{3}^{\prime}\left(\frac{t}{t_{0}}\right)^{\gamma}, & \text { если } \gamma<1,\end{cases}
\end{aligned}
$$

для всех $0<t \leqslant t_{0}$ и некоторых положительных постоянных $c_{1}, c_{1}^{\prime}, c_{2}, c_{2}^{\prime}$, $c_{3}, c_{3}^{\prime}$.

ДокАЗАТЕЛЬСтво. Пусть $\gamma>1$ и $0<t<t_{0}$. В соответствии с нашим предположением имеем

$$
M(t)=\int_{\left\{t / s \leqslant t_{0}\right\}} R\left(\frac{t}{s}\right) e^{-s} d s+R\left(t_{0}\right)\left(1-e^{-t / t_{0}}\right) .
$$

Заметим, что при $0<t<t_{0}$ выполнены неравенства

$$
\frac{t}{2 t_{0}} \leqslant 1-e^{-t / t_{0}} \leqslant \frac{t}{t_{0}} .
$$

Если $R(s) \leqslant A s^{\gamma}$ для всех $0<s<t_{0}$, то

$$
\begin{aligned}
M(t) & \leqslant A t^{\gamma} \int_{t / t_{0}}^{\infty} s^{-\gamma} e^{-s} d s+\frac{R\left(t_{0}\right) t}{t_{0}} \leqslant A s^{\gamma} \int_{t / t_{0}}^{\infty} s^{-\gamma} d s+\frac{R\left(t_{0}\right) t}{t_{0}} \\
& \leqslant \frac{A t^{\gamma}}{\gamma-1}\left(\frac{t}{t_{0}}\right)^{1-\gamma}+\frac{R\left(t_{0}\right) t}{t_{0}}=\frac{t}{t_{0}}\left(R\left(t_{0}\right)+\frac{A t_{0}^{\gamma}}{\gamma-1}\right) .
\end{aligned}
$$

Если же $R(s) \geqslant B s^{\gamma}$ для всех $0<s<t_{0}$, то

$$
M(t) \geqslant \frac{R\left(t_{0}\right)}{2} \frac{t}{t_{0}} .
$$


Предположим, что $0<\gamma<1$ и $0<t<t_{0}$. Снова, если $R(s) \leqslant A s^{\gamma}$ для всех $0<s<t_{0}$, то

$$
\begin{aligned}
M(t) & \leqslant A t^{\gamma} \int_{t / t_{0}}^{\infty} s^{-\gamma} e^{-s} d s+\frac{R\left(t_{0}\right) t}{t_{0}} \leqslant A t^{\gamma} \Gamma(1-\gamma)+R\left(t_{0}\right) \frac{t}{t_{0}} \\
& \leqslant \frac{A t^{\gamma}}{1-\gamma}+R\left(t_{0}\right) \frac{t}{t_{0}}=\frac{A t_{0}^{\gamma}}{1-\gamma}\left(\frac{t}{t_{0}}\right)^{\gamma}+R\left(t_{0}\right) \frac{t}{t_{0}} \\
& \leqslant\left(\frac{t}{t_{0}}\right)^{\gamma}\left(\frac{A t^{\gamma}}{1-\gamma}+R\left(t_{0}\right)\right)
\end{aligned}
$$

Если же $R(s) \geqslant B s^{\gamma}$ для всех $0<s<T$, то

$$
M(t) \geqslant B t^{\gamma} \int_{t / t_{0}}^{\infty} s^{-\gamma} e^{-s} d s \geqslant B t^{\gamma} \int_{1}^{\infty} s^{-\gamma} e^{-s} d s \geqslant\left(\frac{t}{t_{0}}\right)^{\gamma} \frac{B \min \left\{t_{0}, 1\right\}}{e^{2}} .
$$

Наконец, предположим, что $\gamma=1$ и $0<t<t_{0}$. Тогда если $R(s) \leqslant A s^{\gamma}$ для всех $0<s<t_{0}$, то

$$
\begin{aligned}
M(t) & \leqslant A t \int_{t / T}^{\infty} s^{-1} e^{-s} d s+\frac{R(T) t}{T} \\
& =A t\left(\int_{1}^{\infty} s^{-1} e^{-s} d s+\int_{t / t_{0}}^{1} s^{-1} e^{-s} d s\right)+\frac{R\left(t_{0}\right) t}{t_{0}} \\
& \leqslant A t\left(\int_{1}^{\infty} \frac{d s}{s^{2}}+\int_{t / t_{0}}^{1} \frac{d s}{s}\right)+\frac{R\left(t_{0}\right) t}{t_{0}}=\left(A+\frac{R\left(t_{0}\right)}{t_{0}}\right) t\left(\log \frac{t_{0}}{t}+1\right) .
\end{aligned}
$$

Если же $R(s) \geqslant B s^{\gamma}$ для всех $0<s<t_{0}$, то

$$
\begin{aligned}
M(t) & \geqslant B t \int_{t / t_{0}}^{\infty} s^{-1} e^{-s} d s+\frac{R\left(t_{0}\right) t}{2 t_{0}} \\
& \geqslant \frac{B t}{e} \int_{t / t_{0}}^{1} \frac{d s}{s}+\frac{R\left(t_{0}\right) t}{2 t_{0}}=\frac{B t}{e} \log \frac{t_{0}}{t}+\frac{R\left(t_{0}\right) t}{2 t_{0}} \\
& =\frac{B t}{e}\left(\log \frac{t_{0}}{t}+\frac{R\left(t_{0}\right) e}{2 B t_{0}}\right) \geqslant \min \left\{\frac{R\left(t_{0}\right)}{2 t_{0}}, \frac{B}{e}\right\} t\left(\log \frac{t_{0}}{t}+1\right) .
\end{aligned}
$$

Доказательство закончено.

Из доказанных предложений вытекает справедливость теорем 4.3, 4.4 и 4.5.

\section{5. Анализ в $\mathbb{Q}_{p}$ и $\mathbb{Q}_{p}^{n}$}

5.1. Оператор $p$-адической дробной производной. Рассмотрим поле $\mathbb{Q}_{p} \quad p$-адических чисел, наделенное $p$-адической нормой $\|x\|_{p}$ и $p$-адической ультраметрикой $d_{p}(x, y)=\|x-y\|_{p}$. Пусть $\mu_{p}$ - мера Хаара на $\mathbb{Q}_{p}$, нормированная так, что $\mu_{p}\left(\mathbb{Z}_{p}\right)=1$. Пусть $\mathscr{V}_{c}$ - пространство локально постоянных функций на $\mathbb{Q}_{p}$ с компактным носителем, которые будут рассматриваться как пробные функции на $\mathbb{Q}_{p}$. 
Понятие $p$-адической дробной производной, тесно связанное с концепцией p-адической квантовой механики, было введено в работах В.С. Владимирова [57], В. С. Владимирова и И. В. Воловича [58] и В. С. Владимирова, И. В. Воловича и Е.И. Зеленова [59]. В частности, однопараметрическое семейство $\left\{\mathfrak{D}^{\alpha}\right\}_{\alpha>0}$ операторов, называемых операторами дробной производной порядка $\alpha$, было введено в [57].

Напомним, что преобразование Фурье $\mathscr{F}: f \mapsto \widehat{f}$ функции $f$ на самодвойственной локально компактной абелевой группе $\mathbb{Q}_{p}$ определяется равенством

$$
\widehat{f}(\theta)=\int_{\mathbb{Q}_{p}}\langle x, \theta\rangle f(x) d \mu_{p}(x),
$$

где $x, \theta \in \mathbb{Q}_{p}$,

$$
\langle x, \theta\rangle=\exp (2 \pi \sqrt{-1}\{x \theta\})
$$

и $\{x \theta\}$ - дробная часть $p$-адического числа $x \theta($ cp. с $(2.36))$. Известно, что $\mathscr{F}-$ линейный изоморфизм пространства $\mathscr{V}_{c}$ на себя.

ОпРЕДЕлЕниЕ 5.1. Оператор $\left(\mathfrak{D}^{\alpha}, \mathscr{V}_{c}\right), \alpha>0$, определяется через преобразование Фурье на локально компактной абелевой группе $\mathbb{Q}_{p}$ равенством

$$
\widehat{\mathfrak{D}^{\alpha}} f(\xi)=\|\xi\|_{p}^{\alpha} \widehat{f}(\xi), \quad \xi \in \mathbb{Q}_{p} .
$$

Вышеупомянутыми авторами показано, что оператор $\left(\mathfrak{D}^{\alpha}, \mathscr{V}_{c}\right)$ можно записать в виде сингулярного интегрального оператора типа Римана-Лиувилля:

$$
\mathfrak{D}^{\alpha} f(x)=\frac{p^{\alpha}-1}{1-p^{-\alpha-1}} \int_{\mathbb{Q}_{p}} \frac{f(x)-f(y)}{\|x-y\|_{p}^{1+\alpha}} d \mu_{p}(y) .
$$

Цель этого пункта, в частности, состоит в том, чтобы показать, что оператор $\left(\mathfrak{D}^{\alpha}, \mathscr{V}_{c}\right)$ в действительности является ограничением на $\mathscr{V}_{c}$ подходящего изотропного лапласиана. Мы используем следующую функцию распределения расстояний:

$$
\sigma_{\alpha}(r)=\exp \left(-\left(\frac{p}{r}\right)^{\alpha}\right)
$$

Обозначим $\left\{P_{\alpha}^{t}\right\}$ изотропную полугруппу, связанную с тройкой $\left(d_{p}, \mu_{p}, \sigma_{\alpha}\right)$, и пусть $\mathscr{L}_{\alpha}-$ соответствующий лапласиан.

Теорема 5.2. Для любого $\alpha>0$ имеем

$$
\left(\mathscr{L}_{\alpha}, \mathscr{V}_{c}\right)=\left(\mathfrak{D}^{\alpha}, \mathscr{V}_{c}\right)
$$

ДокАЗАтЕЛЬСтво. По теореме 3.12 для любого $f \in \mathscr{V}_{c}$ имеем

$$
\mathscr{L}_{\alpha} f(x)=\int_{\mathbb{Q}_{p}}(f(x)-f(y)) J_{\alpha}(x, y) d \mu_{p}(y),
$$

где

$$
J_{\alpha}(x, y)=\int_{d_{*}(x, y)}^{\infty} \frac{s^{-2} d s}{\mu_{p}\left(B_{s}^{*}(x)\right)} .
$$


Как в примере 2.24, имеем

$$
d_{*}(x, y)=\left(\frac{\|x-y\|_{p}}{p}\right)^{\alpha},
$$

откуда

$$
B_{s}^{*}(x)=B_{p s^{1 / \alpha}}(x)
$$

Замена $r=p s^{1 / \alpha}$ дает

$$
J_{\alpha}(x, y)=p^{\alpha} \int_{\|x-y\|_{p}}^{\infty} \frac{\alpha r^{-\alpha-1} d r}{\mu_{p}\left(B_{r}(x)\right)} .
$$

Поскольку множество значений метрики $\|x-y\|_{p}$ есть $\left\{p^{n}\right\}_{n \in \mathbb{Z}}$, из (2.37) получаем, что

$$
\mu_{p}\left(B_{r}(x)\right)=p^{n}, \quad \text { если } p^{n} \leqslant r<p^{n+1},
$$

откуда следует, что при $\|x-y\|_{p}=p^{k}$

$$
\begin{aligned}
\int_{p^{k}}^{\infty} \frac{\alpha r^{-\alpha-1} d r}{\mu_{p}\left(B_{r}(x)\right)} & =\sum_{n \geqslant k} \int_{p^{n}}^{p^{n+1}} \frac{\alpha r^{-\alpha-1} d r}{\mu_{p}\left(B_{r}(x)\right)} \\
& =\sum_{n \geqslant k} \int_{p^{n}}^{p^{n+1}} \frac{-d r^{-\alpha}}{p^{n}}=\sum_{n \geqslant k} \frac{1}{p^{n}}\left(\frac{1}{p^{n \alpha}}-\frac{1}{p^{(n+1) \alpha}}\right) \\
& =\left(1-\frac{1}{p^{\alpha}}\right) \sum_{n \geqslant k} \frac{1}{p^{n(\alpha+1)}}=\left(1-\frac{1}{p^{\alpha}}\right) \frac{p^{-k(\alpha+1)}}{1-p^{-(\alpha+1)}} \\
& =\frac{1-p^{-\alpha}}{1-p^{-(\alpha+1)}}\left(\frac{1}{p^{k}}\right)^{\alpha+1}=\frac{1-p^{-\alpha}}{1-p^{-(\alpha+1)}}\left(\frac{1}{\|x-y\|_{p}}\right)^{\alpha+1} .
\end{aligned}
$$

Отсюда получаем тождество

$$
J_{\alpha}(x, y)=\frac{p^{\alpha}-1}{1-p^{-\alpha-1}} \frac{1}{\|x-y\|_{p}^{\alpha+1}},
$$

которое ввиду (5.1) завершает доказательство теоремы.

Ядро теплопроводности для полугруппы $\left\{P_{\alpha}^{t}\right\}$ было изучено в примере 2.24 . Мы сформулируем здесь этот результат в виде теоремы.

Теорема 5.3. Полугруппа $\left\{P_{\alpha}^{t}\right\}$ допускает непрерывную переходную плотность $p_{\alpha}(t, x, y)$ относительно меръ Хаара $\mu_{p}$, которая удовлетворяет для всех $t>0$ и $x, y \in \mathbb{Q}_{p}$ оценке

$$
p_{\alpha}(t, x, y) \simeq \frac{t}{\left(t^{1 / \alpha}+\|x-y\|_{p}\right)^{1+\alpha}} .
$$

Верхняя оценка в (5.6) была также получена другим методом А. Н. Кочубеем [39; гл. 4.1, лемма 4.1]. 
ТеОрема 5.4. Полугруппа $\left\{P_{\alpha}^{t}\right\}$ является невозвратной тогда и только тогда, когда $\alpha<1$. В случае невозвратности ее функиия Грина $g_{\alpha}$ явно задается равенством

$$
g_{\alpha}(x, y)=\frac{1-p^{-\alpha}}{1-p^{\alpha-1}}\|x-y\|_{p}^{\alpha-1} .
$$

Формула (5.7) для фундаментального решения оператора $\mathfrak{D}_{\alpha}$, действующего в пространстве $\mathscr{V}_{c}^{\prime}$ распределений Брюа, была получена В. С. Владимировым [57; теорема 1] и А. Н. Кочубеем [39; гл. 2.2].

ДокАзАТЕльство. То, что неравенство $\alpha<1$ равносильно невозвратности, было показано в примере 2.30. Предполагая, что $\alpha<1$, в силу (2.45) получаем

$$
g_{\alpha}(x, y)=\int_{d_{*}(x, y)}^{\infty} \frac{d s}{\mu_{p}\left(B_{s}^{*}(x)\right)}=\frac{1}{p^{\alpha}} \int_{\|x-y\|_{p}}^{\infty} \frac{\alpha r^{\alpha-1} d r}{\mu_{p}\left(B_{r}(x)\right)} .
$$

Полагая $\|x-y\|_{p}=p^{k}$ и используя (5.4), получаем

$$
\begin{aligned}
g_{\alpha}(x, y) & =\frac{1}{p^{\alpha}} \sum_{n \geqslant k} \int_{p^{n}}^{p^{n+1}} \frac{d r^{\alpha}}{p^{n}}=\frac{1}{p^{\alpha}} \sum_{n \geqslant k} \frac{1}{p^{n}}\left(p^{(n+1) \alpha}-p^{n \alpha}\right) \\
& =\frac{1-p^{-\alpha}}{1-p^{\alpha-1}} p^{(\alpha-1) k},
\end{aligned}
$$

что завершает доказательство теоремы.

Обозначим через $\mathscr{L}_{\alpha, q}$ генератор полугруппы $\left\{P_{\alpha}^{t}\right\}$, действующей в $L^{q}\left(\mu_{p}\right)$, $1 \leqslant q<\infty$. Применяя следствие 3.9 и теорему 3.13 , получаем следующее.

Теорема 5.5. Для любого $\alpha>0 u 1 \leqslant q<\infty$ имеем

$$
\operatorname{spec} \mathscr{L}_{\alpha, q}=\left\{p^{\alpha k}: k \in \mathbb{Z}\right\} \cup\{0\} .
$$

Каждое $\lambda_{k}=p^{\alpha k}$ является собственным значением бесконечной кратности.

ДокАЗАтельство. Нам нужно только показать, что кратность числа $\lambda_{k}$ бесконечна. В общей постановке теоремы 3.8 и следствия 3.9 некоторые собственные значения вполне могут иметь конечную кратность, а некоторые нет. Действительно, каждый шар $B$ с минимальным положительным $d_{*}$-радиусом $\rho$ порождает конечномерное собственное пространство $\mathscr{H}_{B}$, состоящее из собственных функций с собственным значением $1 / \rho$. Следовательно, собственное значение $1 / \rho$ имеет конечную кратность тогда и только тогда, когда имеется только конечное число различных шаров $d_{*}$-радиуса $\rho$.

В настоящей постановке в $\mathbb{Q}_{p}$ имеется бесконечно много непересекающихся шаров одного и того же радиуса $\rho$, так как все они получаются сдвигами одного такого шара. Таким образом, все собственные значения имеют бесконечную кратность. Теорема доказана.

Пусть $\left\{X_{t}\right\}$ - марковский процесс на $\mathbb{Q}_{p}$, управляемый марковской полугруппой $\left\{P_{\alpha}^{t}\right\}_{t>0}$. Полугруппа инвариантна относительно сдвигов, откуда следует, что процесс обладает независимыми и стационарными приращениями. Для 
любых заданных $\gamma>0$ и $t>0$ рассмотрим момент порядка $\gamma$ процесса $\mathscr{X}_{t}$, определенного в терминах $p$-адического расстояния $d_{p}(x, y)$ :

$$
\mathscr{M}_{\gamma}(t)=\mathrm{E}\left(\left\|\mathscr{X}_{t}\right\|_{p}^{\gamma}\right)
$$

где E - математическое ожидание относительно вероятностной меры на пространстве траекторий процесса, начинающегося в 0. Применяя теорему 4.3 и используя соотношение $(5.3)$ между $d_{*}$ и $\|\cdot\|_{p}$, получаем следующие оценки.

Теорема 5.6. Момент $\mathscr{M}_{\gamma}(t)$ конечен тогда и толъко тогда, когда $\gamma<\alpha$. В этом случае

$$
\frac{\kappa t^{\gamma / \alpha}}{\alpha-\gamma} \leqslant \mathscr{M}_{\gamma}(t) \leqslant \frac{\alpha t^{\gamma / \alpha}}{\alpha-\gamma}
$$

для некоторого $\kappa>0$.

5.2. Сферически симметричные марковские полугруппы. Пусть $\left\{P^{t}\right\}_{t \geqslant 0}$ - симметричная инвариантная относительно сдвигов марковская полугруппа на аддитивной абелевой группе $\mathbb{Q}_{p}$. Эта полугруппа действует в $C_{0}\left(\mathbb{Q}_{p}\right)$ банаховом пространстве непрерывных функций, обращающихся в нуль на $\infty$. Следовательно, существует слабо непрерывная сверточная полугруппа $\left\{p_{t}\right\}_{t>0}$ симметричных вероятностных мер на $\mathbb{Q}_{p}$ такая, что

$$
P^{t} f(x)=p_{t} * f(x)
$$

Так как вероятностные меры $p_{t}$ симметричны, выполняется следующее тождество, которое является основным в теории безгранично делимых распределений:

$$
\widehat{p_{t}}(\zeta)=\exp (-t \Psi(\zeta))
$$

где $\Psi: \mathbb{Q}_{p} \rightarrow \mathbb{R}_{+}$- отрицательно определенная симметричная функция на $\mathbb{Q}_{p}$. По формуле Леви-Хинчина выполняется равенство

$$
\Psi(\zeta)=\int_{\mathbb{Q}_{p} \backslash\{0\}}(1-\operatorname{Re}\langle x, \zeta\rangle) d \mathfrak{J}(x),
$$

где $\mathfrak{J}$ - симметричная мера Радона на $\mathbb{Q}_{p} \backslash\{0\}$, т. е. мера Леви, связанная с отрицательно определенной функцией $\Psi$ (см. подробности в книге К. Берга и Г. Форста [10]).

ОПРЕДЕЛЕНИЕ 5.7. Для любого $a \in \mathbb{Q}_{p}$ такого, что $\|a\|_{p}=1$, определим оператор вращения $\theta_{a}: \mathbb{Q}_{p} \rightarrow \mathbb{Q}_{p}$ посредством $\theta_{a}(x)=a x$. Будем говорить, что описанная выше марковская полугруппа $\left\{P^{t}\right\}$ инвариантна относительно вращений, если

$$
\theta_{a}\left(p_{t}\right)=p_{t} \quad \text { для всех } a \in \mathbb{Q}_{p} \text { таких, что }\|a\|_{p}=1 .
$$

Пусть $\mathscr{L}$ - генератор полугруппы $\left\{P^{t}\right\}$, т. е. $P^{t}=\exp (-t \mathscr{L})$. Легко видеть, что (5.9) равносильно тождеству $\theta_{a} \circ \mathscr{L}=\mathscr{L} \circ \theta_{a}$. В этом случае будем также говорить, что $\mathscr{L}$ инвариантен относительно вращений. По построению любая изотропная марковская полугруппа $\left\{P^{t}\right\}$, определенная на ультраметрическом 
пространстве с мерой $\left(\mathbb{Q}_{p}, d_{p}, \mu_{p}\right)$, инвариантна относительно вращений. Как мы увидим, класс всех изотропных марковских полугрупп действительно является собственным подмножеством класса инвариантных относительно вращений марковских полугрупп.

Предположим, что полугруппа $\left\{P^{t}\right\}$ инвариантна относительно вращений. Тогда для всех $a$ таких, что $\|a\|_{p}=1$, имеем

$$
\Psi(a \zeta)=\Psi(\zeta) \quad \text { и } \quad \theta_{a}(\mathfrak{J})=\mathfrak{J} .
$$

Поскольку мера Хаара $\mu_{p}$ каждой сферы строго положительна, из (5.9) и (5.10) следует, что меры $p_{t}$ и $\mathfrak{J}$ абсолютно непрерывны относительно $\mu_{p}$ и имеют плотности $p_{t}(x)$ и $J(x)$, зависящие только от $\|x\|_{p}$. То же верно для функции $\Psi$, так что

$$
J(x)=\mathfrak{j}\left(\|x\|_{p}\right) \quad \text { и } \quad \Psi(\zeta)=\psi\left(\|\zeta\|_{p}\right) .
$$

Все вышесказанное показывает, что для генератора $\mathscr{L}$ полугруппы $\left\{P^{t}\right\}$ имеем $\mathscr{V}_{c} \subset \operatorname{dom}_{\mathscr{L}}$ и

$$
\mathscr{L} u=\psi(\mathfrak{D}) u, \quad u \in \mathscr{V}_{c},
$$

где $\mathfrak{D}=\mathfrak{D}^{1}$ - оператор дробной производной порядка $\alpha=1$, который мы отождествляем с изотропным лапласианом $\mathscr{L}_{1}$ по теореме 5.2.

Из (5.11) и (5.2) следует, что собственные функции $\left\{f_{C}: C \in \mathscr{K}\right\}$ оператоpa $\left(\mathscr{L}, \mathscr{V}_{c}\right)$ в $L^{2}$ образуют полную систему, как описано в теореме 3.8 . Каждому шару $B$ радиуса $p^{m}$ ставится в соответствие $(p-1)$-мерное собственное пространство $\mathscr{H}_{B}$, натянутое на функции $f_{C}$, где $C$ пробегает все шары, являющиеся последователями шара $B$, и соответствующее собственное значение равно

$$
\lambda(m)=\psi\left(p^{-m+1}\right) .
$$

Пусть $\{a(m)\}_{m \in \mathbb{Z}}-$ последовательность вещественных чисел, удовлетворяющих условиям

$$
a(m) \geqslant a(m+1), \quad a(+\infty)=0 \quad \text { и } \quad 0<a(-\infty)=W \leqslant+\infty .
$$

Определим последовательность $\{\lambda(m)\}_{m \in \mathbb{Z}}$ равенствами

$$
\lambda(m)=a(m)-(p-1)^{-1}\{a(m+1)-a(m)\} .
$$

Теорема 5.8. Последовательность $\{\lambda(m)\}_{m \in \mathbb{Z}}$ вещественных чисел представляет собой спектр spec $\mathscr{L}$ инвариантного относительно вращений лапласиана $\mathscr{L}$ на $\mathbb{Q}_{p}$ тогда и только тогда, когда она задана равенствами (5.13) с последовательностью а $(m)$, удовлетворяющей условиям (5.12).

ДоказАтельство. Рассмотрим инвариантный относительно вращений лапласиан $\mathscr{L}=\psi(\mathfrak{D})$. Вычислим неотрицательно определенную функцию $\Psi(\zeta)=$ $\psi\left(\|\zeta\|_{p}\right)$, связанную с $\mathscr{L}$. Имеем

$$
\begin{aligned}
\psi\left(\|\zeta\|_{p}\right) & =\int_{\mathbb{Q}_{p} \backslash\{0\}}(1-\operatorname{Re}\langle x, \zeta\rangle) \mathfrak{j}\left(\|x\|_{p}\right) d \mu_{p}(x) \\
& =\sum_{k \in \mathbb{Z}} \mathfrak{j}\left(p^{k}\right) \int_{\left\{x:\|x\|_{p}=p^{k}\right\}}(1-\operatorname{Re}\langle x, \zeta\rangle) d \mu_{p}(x) .
\end{aligned}
$$


Согласно В. С. Владимирову [57; пример 4] имеем

$$
\int_{\left\{x:\|x\|_{p}=p^{k}\right\}}\langle x, \zeta\rangle d \mu_{p}(x)= \begin{cases}p^{k}-p^{k-1}, & \text { если }\|\zeta\|_{p} \leqslant p^{-k} \\ -p^{k-1}, & \text { если }\|\zeta\|_{p}=p^{-k+1} \\ 0, & \text { если }\|\zeta\|_{p} \geqslant p^{-k+2}\end{cases}
$$

В частности,

$$
\int_{\left\{x:\|x\|_{p}=p^{k}\right\}} d \mu_{p}(x)=p^{k}-p^{k-1} .
$$

Пусть $\|\zeta\|_{p}=p^{-m+1}$; тогда приведенные выше вычисления дают

$$
\psi\left(p^{-m+1}\right)=\mathfrak{j}\left(p^{m}\right) p^{m}+\left(1-p^{-1}\right) \sum_{k \geqslant m+1} \mathfrak{j}\left(p^{k}\right) p^{k} .
$$

Определим невозрастающую последовательность $\{a(m)\}_{m \in \mathbb{Z}}$ равенствами

$$
a(m)=\left(1-p^{-1}\right) \sum_{k \geqslant m} \mathfrak{j}\left(p^{k}\right) p^{k}=\left(1-p^{-1}\right) \int_{\left\{x:\|x\|_{p} \geqslant p^{m}\right\}} \mathfrak{j}\left(\|x\|_{p}\right) d \mu_{p}(x) .
$$

В силу (5.15) равенства (5.14) примут следующий вид:

$$
\begin{aligned}
\psi\left(p^{-m+1}\right) & =\frac{p}{p-1}(a(m)-a(m+1))+a(m+1) \\
& =a(m)-(p-1)^{-1}(a(m+1)-a(m)) .
\end{aligned}
$$

Пусть $\lambda(m)$ - собственное значение лапласиана $\left(\psi(\mathfrak{D}), \mathscr{V}_{c}\right)$, соответствующее шару $B$ радиуса $p^{m}$. Тогда $\lambda(m)=\psi\left(p^{-m+1}\right)$ и тождество (5.16) дает желаемый результат, а именно равенство (5.13).

Обратно, для заданной последовательности $\{a(m)\}$, удовлетворяющей $(5.12)$, определим последовательность $\{\lambda(m)\}$ равенствами (5.13) и положим

$$
\begin{aligned}
& \Psi(\xi)=\psi\left(\|\xi\|_{p}\right), \quad \text { где } \psi\left(p^{m}\right)=\lambda(-m+1), \\
& J(x)=\mathfrak{j}\left(\|x\|_{p}\right), \quad \text { где } \mathfrak{j}\left(p^{m}\right)=\frac{a(m)-a(m+1)}{p^{m}-p^{m-1}} .
\end{aligned}
$$

Непосредственно показывается, что

$$
\Psi(\zeta)=\int_{\mathbb{Q}_{p} \backslash\{0\}}(1-\operatorname{Re}\langle x, \zeta\rangle) J(x) d \mu_{p}(x),
$$

откуда следует, что $\Psi$ - неотрицательно определенная функция. Следовательно, функция $\exp (-t \Psi)$ положительно определена, откуда следует, что она является преобразованием Фурье вероятностной меры $p_{t}$. Ясно, что $\left\{p_{t}\right\}_{t>0}-$ слабо непрерывная сверточная полугруппа вероятностных мер. По построению каждая мера $p_{t}$ инвариантна относительно вращений. Наконец, мы определяем инвариантную относительно сдвигов марковскую полугруппу равенством $P^{t} f=f * p_{t}$. Теорема доказана. 
СледСтвиЕ 5.9. Во введенных выше обозначениях следующие утверждения равносильны:

(1) последовательность $\lambda(m)$ невозрастающая;

(2) последовательность $\psi\left(p^{m}\right)$ неубывающая;

(3) последовательность $\mathfrak{j}\left(p^{m}\right)$ невозрастающая.

В частности, если последовательность а $(m)$ выпуклая, то выполняется каждое из равносильных свойств (1)-(3).

ДоказАтельство. Эквивалентность $(1) \Leftrightarrow(2)$ является следствием соотношения $\lambda(m)=\psi\left(p^{-m+1}\right)$. Чтобы доказать, что $(1) \Leftrightarrow(3)$, применяем $(5.17)$ и получаем

$$
\lambda(m)-\lambda(m+1)=\left(p^{m}-p^{m-1}\right)\left(\mathfrak{j}\left(p^{m}\right)-\mathfrak{j}\left(p^{m+1}\right)\right) .
$$

Отсюда следует эквивалентность (1) $\Leftrightarrow(2) \Leftrightarrow(3)$. Наконец, (5.13) и выпуклость $a(m)$ дают (1). Следствие доказано.

Далее рассмотрим строгую монотонность.

СлеДСтвиЕ 5.10. Следующие утверждения равносильны:

(i) последовательность $\lambda(m)$ строго убъвает и $\lambda(-\infty)=+\infty$;

(ii) последовательность $\psi\left(p^{m}\right)$ строго возрастает $u \psi(+\infty)=+\infty$;

(iii) последовательность $\mathfrak{j}\left(p^{m}\right)$ строго убъвает $u$

$$
\int \mathfrak{j}\left(\|x\|_{p}\right) d \mu_{p}(x)=+\infty
$$

(iv) соответствующая инвариантная относительно вращений марковская полугруппа $\left\{P^{t}\right\}$ изотропна.

$B$ частности, если последовательность $а(m)$ строго выпукла и $а(-\infty)=$ $+\infty$, то выполняется каждое из равносильных свойств (i)-(iv).

ДокАзАтельство. Эквивалентность (i) $\Leftrightarrow$ (ii) $\Leftrightarrow$ (iii) следует из таких же рассуждений, как в доказательстве следствия 5.9. Выпуклость $a(m)$ вместе с равенством $a(-\infty)=+\infty$ влекут (i) с помощью тех же рассуждений. Остается показать, что (iv) $\Leftrightarrow$ (ii).

Предположим, что $\left\{P^{t}\right\}$ - изотропная марковская полугруппа, построенная в (1.3)-(1.8). Эта полугруппа допускает непрерывную переходную плотность $p(t, x, y)=p_{t}(x-y)$ относительно меры Хаара $\mu_{p}$; функция $p_{t}$ задается равенством

$$
p_{t}(y)=\int_{0}^{\infty} q_{s}(y) d \sigma^{t}(s), \quad \text { где } q_{s}(y)=\frac{1}{\mu_{p}\left(B_{s}(0)\right)} \mathbf{1}_{B_{s}(0)}(y) .
$$

Чтобы найти преобразование Фурье $\widehat{p}_{t}(\xi)$, рассуждаем следующим образом. Шар $B_{s}(0), p^{k} \leqslant s<p^{k+1}$, является компактной подгруппой $p^{-k} \mathbb{Z}_{p}$ группы $\mathbb{Q}_{p}$, откуда следует, что мера $\omega_{s}=q_{s} \mu_{p}$ совпадает с нормированной мерой Хаара этой компактной подгруппы. Поскольку для любой локально компактной абелевой группы преобразование Фурье нормированной меры Хаара любой компактной подгруппы является индикатором ее аннулирующей группы, а в нашем частном случае аннулятором группы $p^{-k} \mathbb{Z}_{p}$ является группа $p^{k} \mathbb{Z}_{p}$, получаем

$$
\widehat{\omega_{s}}(\xi)=\mathbf{1}_{p^{k} \mathbb{Z}_{p}}(\xi)=\mathbf{1}_{\left[0, p^{-k}\right]}\left(\|\xi\|_{p}\right), \quad \text { где } p^{k} \leqslant s<p^{k+1} .
$$


Следовательно, когда $\|\xi\|_{p}=p^{-l}$, имеем

$$
\widehat{p}_{t}(\xi)=\sum_{k: k \leqslant l}\left(\sigma^{t}\left(p^{k+1}\right)-\sigma^{t}\left(p^{k}\right)^{t}\right)=\sigma^{t}\left(p^{l+1}\right)=\exp \left(-t \psi\left(\|\xi\|_{p}\right)\right),
$$

откуда

$$
\psi\left(p^{-l}\right)=\log \frac{1}{\sigma\left(p^{l+1}\right)} .
$$

Согласно (1.5) последовательность $\sigma\left(p^{l}\right)$ предполагается строго возрастающей и стремящейся к нулю при $l \rightarrow-\infty$. Таким образом, $\psi\left(p^{m}\right)$ такая, как утверждается в (ii). Обратно, если дана строго возрастающая последовательность $\psi\left(p^{m}\right)$, как в (ii), мы определяем строго возрастающую последовательность

$$
\sigma\left(p^{m}\right)=\exp \left(-\psi\left(p^{-m+1}\right)\right)
$$

Пусть $\sigma:[0, \infty) \rightarrow[0,1)$ - любая возрастающая биекция, принимающая значения $\sigma\left(p^{m}\right)$ в точках $p^{m}$. Определим функцию $p_{t}(y)$ равенством (5.18). Так как $\sigma(+\infty)=1$, это вероятностная плотность относительно $\mu_{p}$. Ясно, что $\left\{p_{t}\right\}_{t>0}-$ слабо непрерывная сверточная полугруппа вероятностных мер на $\mathbb{Q}_{p}$. Более того, каждая $p_{t}$ инвариантна относительно вращений по построению. Таким образом, полугруппа $P^{t} f=f * p_{t}$ является изотропной. Следствие доказано.

ЗАмечание 5.11. В [2] С. Альбеверио и В. Карвовски начинали с после-

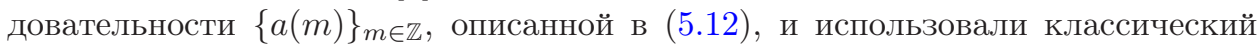
подход обратных и прямых уравнений Колмогорова для построения марковской полугруппы $\left\{P^{t}\right\}$ на ультраметрическом пространстве с мерой $\left(\mathbb{Q}_{p}, d_{p}, \mu_{p}\right)$. В частности, они показали (см. [2; теорема 3.29$]$ ), что лапласиан $\mathscr{L}$ этой полугруппы обладает чисто точечным спектром $\{\lambda(m)\}$, определенным в $(5.13)$, и $\lambda(m)$-собственное пространство натянуто на функции $f_{B}$, где $B$ пробегает все шары радиуса $p^{m-1}$. Наша теорема 5.8 показывает, что на самом деле класс марковских полугрупп, построенный в [2], совпадает с классом марковских полугрупп, инвариантных относительно вращений.

5.3. Пространства произведений. Пусть $\left\{\left(X_{i}, d_{i}\right)\right\}_{i=1}^{n}-$ конечная последовательность ультраметрических пространств; мы считаем, что все $\left(X_{i}, d_{i}\right)$ сепарабельны и что все шары компактны. Пусть $(X, d)$ - их декартово произведение: $X=X_{1} \times \cdots \times X_{n}$ и для $x=\left(x_{i}\right) \in X$ и $y=\left(y_{i}\right) \in Y$ положим

$$
d(x, y)=\max \left\{d_{i}\left(x_{i}, y_{i}\right): i=1,2, \ldots, n\right\} .
$$

Таким образом, $(X, d)$ - сепарабельное ультраметрическое пространство, все шары в $(X, d)$ компактны и, более того, каждый $d$-шар $B_{r}(a)$ в $X$ является произведением $d_{i}$-шаров $B_{r}^{i}\left(a_{i}\right)$ в $X_{i}$ того же радиуса.

Если на каждом $\left(X_{i}, d_{i}\right)$ задана мера Радона $\mu_{i}$, определим $\mu=\otimes \mu_{i}$ на $(X, d)$. Пусть $\mathscr{V}_{c}$ - множество всех локально постоянных функций на $(X, d)$ с компактными носителями.

Рассмотрим ультраметрическое пространство с мерой $(X, d, \mu)$. Согласно предыдущим пунктам существует богатый класс изотропных марковских полугрупп и соответствующих лапласианов на $(X, d, \mu)$, таких как построенные 
в (1.3)-(1.8). Благодаря структуре произведения пространства $(X, d, \mu)$ можно естественно определить нетривиальный и интересный класс марковских полугрупп и лапласианов, которые не изотропны. А именно, выбирая на каждом $\left(X_{i}, d_{i}, \mu_{i}\right)$ изотропную марковскую полугруппу $\left\{P_{i}^{t}\right\}$, определим марковскую полугруппу $\left\{P^{t}\right\}$ на $(X, d, \mu)$ как тензорное произведение полугрупп $\left\{P_{i}^{t}\right\}$ :

$$
P^{t}=\bigotimes_{i=1}^{n} P_{i}^{t}
$$

Полугруппа $\left\{P^{t}\right\}$ имеет следующее ядро теплопроводности:

$$
p(t, x, y)=\prod_{i=1}^{n} p_{i}\left(t, x_{i}, y_{i}\right)
$$

где $p_{i}$ - ядро теплопроводности полугруппы $\left\{P_{i}^{t}\right\}$.

Генератор $\mathscr{L}$ полугруппы $P^{t}$ можно описать следующим образом: $\mathscr{V}_{c} \subset$ $\operatorname{dom}_{\mathscr{L}}$ и для любого $f \in \mathscr{V}_{c}$ имеем

$$
\mathscr{L} f(x)=\sum_{i=1}^{n} \mathscr{L}_{i} f(x),
$$

где $x=\left(x_{1}, \ldots, x_{n}\right)$ и $\mathscr{L}_{i}$ действует на $x_{i}$. Следовательно,

$$
\mathscr{L} f(x)=\int_{X}(f(x)-f(y)) J(x, d y),
$$

где

$$
J(x, d y)=\sum_{i=1}^{n} J_{i}\left(x_{i}, y_{i}\right) d \mu_{i}\left(y_{i}\right)
$$

и $J_{i}\left(x_{i}, y_{i}\right)$ - ядро генератора $\mathscr{L}_{i}$.

В частности, для каждого $x \in X$ меры $J(x, d y)$ и $\mu(d y)$ не обязательно взаимно абсолютно непрерывны (в случае, когда хотя бы одно из $X_{l}$ совершенно, $J(x, d y)$ сингулярно относительно $\mu$ ), откуда следует, что полугруппа $\left\{P^{t}\right\}$ не обязательно является изотропной марковской полугруппой.

В этой работе мы не собираемся развивать общую теорию пространств произведений. Нашей целью является подробное изучение двух конкретных примеров, связанных с р-адическим анализом.

В первом примере мы рассматриваем лапласиан Владимирова, который хорошо согласуется с вышеописанной общей конструкцией. Во втором примере мы рассматриваем лапласиан Тайблесона, определенный в терминах многомерных ядер Рисса (см. книгу М. Х. Тайблесона [55] и работу Х. Родригеса-Вега и В. А. Зунига-Галиндо [50]). Мы покажем, что лапласиан Тайблесона изотропен. Это позволит нам улучшить оценки ядра теплопроводности из [50] и получить некоторые новые результаты (невозвратность/возвратность, независимость $L^{p}$-спектра от $1 \leqslant p<\infty$, точные оценки моментов соответствующего марковского процесса и т. п.). 
Рассмотрим линейное пространство $\mathbb{Q}_{p}^{n}=\mathbb{Q}_{p} \times \cdots \times \mathbb{Q}_{p}$ над полем $\mathbb{Q}_{p}$ и определим в $\mathbb{Q}_{p}^{n}$ норму

$$
\|z\|_{p}=\max \left\{\left\|z_{i}\right\|_{p}: i=1,2, \ldots, n\right\}
$$

Ясно, что эта норма удовлетворяет ультраметрическому неравенству треугольника (1.1) и является однородной в следующем смысле:

$$
\|a z\|_{p}=\|a\|_{p}\|z\|_{p} \quad \text { для всех } a \in \mathbb{Q}_{p}, z \in \mathbb{Q}_{p}^{n} .
$$

Положим

$$
d_{p}(x, y)=\|x-y\|_{p},
$$

так что $\left(\mathbb{Q}_{p}^{n}, d_{p}\right)$ - ультраметрическое пространство.

Пусть $\mu_{p}=\bigotimes \mu_{p, i}$ - аддитивная мера Хаара на абелевой группе $\mathbb{Q}_{p}^{n}$. Как и ранее, пусть $\mathscr{V}_{c}$ - множество всех локально постоянных функций с компактными носителями на ультраметрическом пространстве $\left(\mathbb{Q}_{p}^{n}, d_{p}\right)$. Напомним, что $\mathscr{V}_{c}$ - всюду плотное подмножество в $L^{2}=L^{2}\left(\mathbb{Q}_{p}^{n}, \mu_{p}\right)$.

5.3.1. Лапласиан Владимирова. Для любого данного $n$-мультииндекса $\alpha=$ $\left(\alpha_{1}, \ldots, \alpha_{n}\right)$ с элементами $\alpha_{i}>0$ определим ультраметрику

$$
d_{p, \alpha}(x, y)=\max \left\{\left\|x_{i}-y_{i}\right\|_{p}^{\alpha_{i}}: i=1,2, \ldots, n\right\},
$$

которую будем обозначать также $\|x-y\|_{p, \alpha}$. В частности, ультраметрика $d_{p}(x, y)$, определенная выше, соответствует случаю $\alpha=(1, \ldots, 1)$. Тождественное отображение

$$
\left(\mathbb{Q}_{p}^{n}, d_{p, \alpha}\right) \rightarrow\left(\mathbb{Q}_{p}^{n}, d_{p}\right)
$$

является гомеоморфизмом, но оно не билипшицево, если только не выполняется $\alpha_{i}=1$ для всех $i$. Этот факт играет существенную роль в изучении класса лапласианов, которые вводятся далее как частный случай лапласианов (5.19).

ОПРЕДЕЛЕНИЕ 5.12. Пусть $\alpha=\left(\alpha_{1}, \ldots, \alpha_{n}\right)$. Для любой функции $f \in \mathscr{V}_{c}$ определим оператор

$$
\mathfrak{V}^{\alpha} f(x)=\sum_{i=1}^{n} \mathfrak{D}_{x_{i}}^{\alpha_{i}} f(x),
$$

где $x=\left(x_{1}, \ldots, x_{n}\right)$, а $\mathfrak{D}_{x_{i}}^{\alpha_{i}}-p$-адическая дробная производная порядка $\alpha_{i}$, действующая на $x_{i}$.

Оператор $\mathfrak{V}^{\alpha}$ на $\mathbb{Q}_{p}^{3}$ с $\alpha=(2,2,2)$ был введен В. С. Владимировым [57] в качестве аналога классического оператора Лапласа в $\mathbb{R}^{3}$. Этот оператор, который мы сокращенно обозначаем через $\mathfrak{V}^{2}$, инвариантен относительно сдвигов и однороден, т. е.

$$
\mathfrak{V}^{2} \tau_{y}(f)=\tau_{y}\left(\mathfrak{V}^{2} f\right), \quad \text { где } \tau_{y} f(x)=f(x+y),
$$

и

$$
\mathfrak{V}^{2} \theta_{a}(f)=\|a\|_{p}^{2} \theta_{a}\left(\mathfrak{V}^{2} f\right), \quad \text { где } \theta_{a} f(x)=f\left(a x_{1}, a x_{2}, a x_{3}\right) .
$$


Следовательно, функция Грина $g(x, y)$ оператора $\mathfrak{V}^{2}$ на $\mathbb{Q}_{p}^{3}$ также инвариантна относительно сдвигов и однородна:

$$
g(x, y)=g(x-z, y-z), \quad g(a x, a y)=\frac{g(x, y)}{\|a\|_{p}}, \quad a \in \mathbb{Q}_{p} .
$$

В частности, полагая $\mathfrak{E}(x)=g(x, 0)$, получаем для всех ненулевых $a \in \mathbb{Q}_{p}$ тождество

$$
\mathfrak{E}(a, a, a)=\frac{\mathfrak{E}(1,1,1)}{\|a\|_{p}} .
$$

Это тождество было отмечено в [57]. Оно дает представление о том, как функция Грина оператора $\mathfrak{V}^{2}$ (в терминологии В. С. Владимирова - фундаментальное решение уравнения $\mathfrak{V}^{2} \mathfrak{E}=\delta$ ) ведет себя на бесконечности в нуле. Ниже, в предложении 5.15, мы докажем, что для всех ненулевых $a=\left(a_{1}, a_{2}, a_{3}\right) \in \mathbb{Q}_{p}^{3}$ справедливо

$$
\mathfrak{E}\left(a_{1}, a_{2}, a_{3}\right) \simeq \frac{1}{\|a\|_{p}}
$$

По существу мы докажем аналогичную оценку для более общих операторов $\mathfrak{V}^{\alpha}$ без свойства однородности. Начнем с перечисления некоторых свойств оператора $\left(\mathfrak{V}^{\alpha}, \mathscr{V}_{c}\right)$ из определения 5.12, которые вытекают непосредственно из соответствующих свойств “одномерных лапласианов" $\mathfrak{D}^{\alpha_{i}}$.

1. Оператор $\left(\mathfrak{V}^{\alpha}, \mathscr{V}_{c}\right)$ является неотрицательно определенным симметричным оператором.

2. Оператор $\left(\mathfrak{V}^{\alpha}, \mathscr{V}_{c}\right)$ допускает полную систему собственных функций с компактными носителями. В частности, оператор $\left(\mathfrak{V}^{\alpha}, \mathscr{V}_{c}\right)$ является существенно самосопряженным.

3. Полугруппа $\exp \left(-t \mathfrak{V}^{\alpha}\right)$ симметрична и является марковской полугруппой. $\mathrm{y}$ нее есть ядро теплопроводности $p_{\alpha}(t, x, y)$, имеющее следующий вид:

$$
p_{\alpha}(t, x, y)=\prod_{i=1}^{n} p_{\alpha_{i}}\left(t, x_{i}, y_{i}\right)
$$

4. Полугруппа $\exp \left(-t \mathfrak{V}^{\alpha}\right)$ является невозвратной тогда и только тогда, когда

$$
A:=\sum_{i=1}^{n} \frac{1}{\alpha_{i}}>1 \text {. }
$$

5. Для всех $f \in \mathscr{V}_{c}$ справедливо равенство

$$
\mathfrak{V}^{\alpha} f(x)=\int_{\mathbb{Q}_{p}^{n}}(f(x)-f(y)) J_{\alpha}(x, d y),
$$

где

$$
J_{\alpha}(x, d y)=\sum_{i_{1}}^{n} J_{\alpha_{i}}\left(x_{i}-y_{i}\right) d \mu_{p, i}\left(y_{i}\right)
$$

и

$$
J_{\alpha_{i}}\left(x_{i}-y_{i}\right)=\frac{p^{\alpha_{i}}-1}{1-p^{-\alpha_{i}-1}} \frac{1}{\left\|x_{i}-y_{i}\right\|_{p}^{1+\alpha_{i}}} \text {. }
$$


В частности, полугруппа $\exp \left(-t \mathfrak{V}^{\alpha}\right)$ в случае $n>1$ не является изотропной марковской полугруппой.

Заметим, что благодаря групповой структуре $\mathbb{Q}_{p}^{n}$ функции $(x, y) \mapsto p_{\alpha}(t, x, y)$ и $(x, y) \mapsto g_{\alpha}(x, y)$ инвариантны относительно сдвигов. Отсюда следует, что, полагая

$$
p_{\alpha}(t, z)=p_{\alpha}(t, z, 0) \quad \text { и } \quad g_{\alpha}(z)=g_{\alpha}(z, 0),
$$

мы получим

$$
p_{\alpha}(t, x, y)=p_{\alpha}(t, x-y) \quad \text { и } \quad g_{\alpha}(x, y)=g_{\alpha}(x-y) .
$$

ПРЕДЛОЖЕНИЕ 5.13. Пусть

$$
A=\sum_{i=1}^{n} \frac{1}{\alpha_{i}}
$$

Тогда ядро теплопроводности удовлетворяет следующей оценке:

$$
p_{\alpha}(t, z) \simeq t^{-A} \prod_{i=1}^{n} \min \left\{1, \frac{t^{1+1 / \alpha_{i}}}{\left\|z_{i}\right\|_{p}^{1+\alpha_{i}}}\right\}
$$

равномерно для всех $t>0 u z \in \mathbb{Q}_{p}^{n}$. В частности, для всех $t>\|z\|_{p, \alpha}$

$$
p_{\alpha}(t, z) \simeq t^{-A}
$$

ДокАЗАТЕЛьство. По теореме 5.3 имеем

$$
p_{\alpha_{i}}\left(t, z_{i}\right) \simeq \frac{t}{\left(t^{1 / \alpha_{i}}+\left\|z_{i}\right\|_{p}\right)^{1+\alpha_{i}}} \simeq \frac{1}{t^{1 / \alpha_{i}}} \min \left\{1, \frac{t^{1+1 / \alpha_{i}}}{\left\|z_{i}\right\|_{p}^{1+\alpha_{i}}}\right\},
$$

откуда следует утверждение.

ПРЕДЛОЖЕНИЕ 5.14. Полугруппа $\exp \left(-t \mathfrak{V}^{\alpha}\right)$ является невозвратной тогда и только тогда, когда $A>1$. Если $A>1$, то для всех $z \in \mathbb{Q}_{p}^{n}$ и некоторого $C_{1}>0$ выполняется неравенство

$$
g_{\alpha}(z) \geqslant C_{1}\left(\frac{1}{\|z\|_{p, \alpha}}\right)^{A-1}
$$

Для любого $\kappa>0$ определим множество

$$
\Omega(\kappa)=\left\{x \in \mathbb{Q}_{p}^{n}: \max _{i}\left\{\left\|x_{i}\right\|_{p}^{\alpha_{i}}\right\} \leqslant \kappa \min _{i}\left\{\left\|x_{i}\right\|_{p}^{\alpha_{i}}\right\}\right\}
$$

Тогда для всех $z \in \Omega(\kappa)$ и некоторой постоянной $C_{2}>0$, зависящей от $\kappa$, выполняется неравенство

$$
g_{\alpha}(z) \leqslant C_{2}\left(\frac{1}{\|z\|_{p, \alpha}}\right)^{A-1}
$$


ДоказАтеЛЬСтво. Критерий невозвратности $A>1$ следует из соотношения $p_{a}(t, x, x) \simeq t^{-A}$. Чтобы доказать нижнюю оценку, воспользуемся (5.23) и запишем

$$
g_{\alpha}(z)=\int_{0}^{\infty} p_{\alpha}(t, z) d t \geqslant \int_{\|z\|_{p, \alpha}}^{\infty} p_{\alpha}(t, z) d t \geqslant C_{1} \int_{\|z\|_{p, \alpha}}^{\infty} t^{-A} d t=c_{1}\left(\frac{1}{\|z\|_{p, \alpha}}\right)^{A-1} .
$$

С другой стороны, имеем

$$
g_{\alpha}(z)=\left(\int_{0}^{\|z\|_{p, \alpha}}+\int_{\|z\|_{p, \alpha}}^{\infty}\right) p_{\alpha}(t, z) d t=: \mathrm{I}+\mathrm{II} .
$$

Чтобы оценить член II, снова воспользуемся (5.23):

$$
\mathrm{II} \simeq \int_{\|z\|_{p, \alpha}}^{\infty} t^{-A} d t \simeq\left(\frac{1}{\|z\|_{p, \alpha}}\right)^{A-1} .
$$

Чтобы оценить член I, воспользуемся (5.22):

$$
\begin{aligned}
\mathrm{I} & \leqslant c \int_{0}^{\|z\|_{p, \alpha}} t^{-A} \prod_{i=1}^{n} \frac{t^{1+1 / \alpha_{i}}}{\left\|z_{i}\right\|_{p}^{1+\alpha_{i}}} d t \\
& =c \int_{0}^{\|z\|_{p, \alpha}} \prod_{i=1}^{n} \frac{1}{\left\|z_{i}\right\|_{p}^{1+\alpha_{i}}} t^{n} d t=c^{\prime} \prod_{i=1}^{n} \frac{1}{\left\|z_{i}\right\|_{p}^{1+\alpha_{i}}}\|z\|_{p, \alpha}^{n+1} .
\end{aligned}
$$

Когда $z \in \Omega(\kappa)$, получаем

$$
\begin{aligned}
\mathrm{I} & \leqslant c^{\prime \prime} \prod_{i=1}^{n} \frac{1}{\left\|z_{i}\right\|_{p}^{1+\alpha_{i}}}\left(\min _{i}\left\{\left\|z_{i}\right\|_{p}^{\alpha_{i}}\right\}\right)^{n+1} \leqslant c^{\prime \prime} \min _{i}\left\{\left\|z_{i}\right\|_{p}^{\alpha_{i}}\right\} \prod_{i=1}^{n} \frac{1}{\left\|z_{i}\right\|_{p}} \\
& =c^{\prime \prime} \min _{i}\left\{\left\|z_{i}\right\|_{p}^{\alpha_{i}}\right\} \prod_{i=1}^{n} \frac{1}{\left(\left\|z_{i}\right\|_{p}^{\alpha_{i}}\right)^{1 / \alpha_{i}}} .
\end{aligned}
$$

Далее,

$$
\prod_{i=1}^{n} \frac{1}{\left(\left\|z_{i}\right\|_{p}^{\alpha_{i}}\right)^{1 / \alpha_{i}}} \leqslant \prod_{i=1}^{n} \frac{1}{\left(\min _{j}\left\{\left\|z_{j}\right\|_{p}^{\alpha_{j}}\right\}\right)^{1 / \alpha_{i}}}=\left(\frac{1}{\min _{j}\left\{\left\|z_{j}\right\|_{p}^{\alpha_{j}}\right\}}\right)^{A}
$$

откуда следует

$$
I \leqslant c^{\prime \prime}\left(\frac{1}{\min _{j}\left\{\left\|z_{j}\right\|_{p}^{\alpha_{j}}\right\}}\right)^{A-1} .
$$

Снова используя тот факт, что $z \in \Omega(\kappa)$, запишем

$$
\left(\frac{1}{\min _{j}\left\{\left\|z_{j}\right\|_{p}^{\alpha_{j}}\right\}}\right)^{A-1} \leqslant\left(\frac{\kappa}{\max _{j}\left\{\left\|z_{j}\right\|_{p}^{\alpha_{j}}\right\}}\right)^{A-1}=c(\kappa)\left(\frac{1}{\|z\|_{p, \alpha}}\right)^{A-1} .
$$

Из полученных верхних оценок интегралов I и II следуют требуемые верхние оценки для $g_{\alpha}(z)$. Предложение доказано. 
ПРЕДЛОЖЕНИЕ 5.15. Пусть $\alpha=\left(\alpha_{1}, \ldots, \alpha_{n}\right)=(\beta, \ldots, \beta)$ есть $n$-мулътииндекс, в котором все элементы равны $\beta$. Предположим, что $(n-1) / 2<$ $\beta<n$. Тогда полугруппа $\exp \left(-t \mathfrak{V}^{\alpha}\right)$ является невозвратной и функиия Грина $g_{\alpha}(z)$ удовлетворяет оценкам

$$
g_{\alpha}(z) \simeq\left(\frac{1}{\|z\|_{p, \alpha}}\right)^{A-1}
$$

для всех $z \in \mathbb{Q}_{p}^{n}$ и некоторых $c_{1}, c_{2}>0$.

Поскольку $A=n / \beta$ и $\|z\|_{p, \alpha}=\|z\|_{p}^{\beta}$, оценка (5.24) равносильна оценке

$$
g_{\alpha}(z) \simeq\left(\frac{1}{\|z\|_{p}}\right)^{n-\beta} .
$$

ДокАЗАтЕльство. Невозвратность следует из предложения 5.14, поскольку $A=n / \beta>1$. То же предложение дает требуемую нижнюю оценку функции Грина. Чтобы доказать оценку сверху, заметим, что лапласиан $\mathfrak{V}^{\alpha}$ однороден, т. e.

$$
\mathfrak{V}^{\alpha} \circ \theta_{a}=\|a\|_{p}^{\beta} \cdot \theta_{a} \circ \mathfrak{V}^{\alpha}
$$

для всех $a \in \mathbb{Q}_{p}$. Отсюда следует, что функция Грина $g_{\alpha}(z)$ также однородна, т. e.

$$
g_{\alpha}(a z)=\|a\|_{p}^{n-\beta} g_{\alpha}(z)
$$

для всех $a \in \mathbb{Q}_{p}$ и $z \in \mathbb{Q}_{p}^{n}$.

Без ограничения общности предположим, что $\|z\|_{p, \alpha}=\left\|z_{1}\right\|_{p}^{\beta}>0$. Тогда

$$
\begin{aligned}
g_{\alpha}(z) & =g_{\alpha}\left(z_{1}\left(1, \frac{z_{2}}{z_{1}}, \ldots, \frac{z_{n}}{z_{1}}\right)\right)=\left\|z_{1}\right\|_{p}^{n-\beta} g_{\alpha}\left(1, \frac{z_{2}}{z_{1}}, \ldots, \frac{z_{n}}{z_{1}}\right) \\
& =\left(\frac{1}{\|z\|_{p, \alpha}}\right)^{A-1} g_{\alpha}\left(1, \frac{z_{2}}{z_{1}}, \ldots, \frac{z_{n}}{z_{1}}\right) \\
& \leqslant\left(\frac{1}{\|z\|_{p, \alpha}}\right)^{A-1} \sup \left\{g_{\alpha}\left(1, x_{2}, \ldots, x_{n}\right): x_{i} \in \mathbb{Z}_{p}\right\} .
\end{aligned}
$$

Далее применим наше предположение $\beta>(n-1) / 2$ и получим из (5.22), что

$$
\begin{aligned}
g_{\alpha}\left(1, x_{2}, \ldots, x_{n}\right) & =\int_{0}^{\infty} p_{\alpha}\left(t,\left(1, x_{2}, \ldots, x_{n}\right)\right) d t \\
& =\left(\int_{0}^{1}+\int_{1}^{\infty}\right) p_{\alpha}\left(t,\left(1, x_{2}, \ldots, x_{n}\right)\right) d t \\
& \leqslant c \int_{0}^{1} t^{-n / \beta} t^{1+1 / \beta} d t+c^{\prime} \int_{1}^{\infty} t^{-n / \beta} d t=c_{2}<\infty
\end{aligned}
$$

откуда следует требуемая верхняя оценка. Предложение доказано. 
5.3.2. Лапласиан Тайблесона. Преобразование Фурье $\mathscr{F}: f \mapsto \widehat{f}$ функции $f$ на локально компактной абелевой группе $\mathbb{Q}_{p}^{n}$ определяется формулой

$$
\widehat{f}(\theta)=\int_{\mathbb{Q}_{p}^{n}}\langle x, \theta\rangle f(x) d \mu_{p}^{n}(x),
$$

где $x=\left(x_{1}, \ldots, x_{n}\right) \in \mathbb{Q}_{p}^{n}, \theta=\left(\theta_{1}, \ldots, \theta_{n}\right) \in\left(\mathbb{Q}_{p}^{n}\right)^{*}=\mathbb{Q}_{p}^{n}$,

$$
\langle x, \theta\rangle=\prod_{k=1}^{n}\left\langle x_{k}, \theta_{k}\right\rangle
$$

и $d \mu_{p}^{n}(x)=d \mu_{p}\left(x_{1}\right) \cdots d \mu_{p}\left(x_{n}\right)$ - мера Хаара на $\mathbb{Q}_{p}^{n}$. Известно, что $\mathscr{F}-$ линейный изоморфизм из $\mathscr{V}_{c}$ на себя, что оправдывает следующее определение (ср. с определением 5.1).

ОпРЕДЕлЕниЕ 5.16. Оператор Тайблесона $\mathfrak{T}^{\alpha}$ для $\alpha>0$ определяется на функциях $f \in \mathscr{V}_{c}$ формулой

$$
\widehat{\mathfrak{T}^{\alpha} f}(\zeta)=\|\zeta\|_{p}^{\alpha} \widehat{f}(\zeta), \quad \zeta \in \mathbb{Q}_{p}^{n} .
$$

Из данного определения вытекает, что $\left(\mathfrak{T}^{\alpha}, \mathscr{V}_{c}\right)$ - существенно самосопряженный и неотрицательно определенный оператор в $L^{2}$. Этот оператор был введен M.X. Тайблесоном [55], а связанная с ним полугруппа $\exp \left(-t \mathfrak{T}^{\alpha}\right)$ изучалась Х. Родригесом-Вега и В. А. Зунига-Галиндо [50]. В частности, было показано, что

$$
\mathfrak{T}^{\alpha} f(x)=\frac{p^{\alpha}-1}{1-p^{-\alpha-n}} \int_{\mathbb{Q}_{p}^{n}} \frac{f(x)-f(y)}{\|x-y\|_{p}^{\alpha+n}} d \mu_{p}^{n}(y) .
$$

Из равенства (5.26) вытекает, что оператор $\left(-\mathfrak{T}^{\alpha}, \mathscr{V}_{c}\right)$ удовлетворяет принципу максимума, откуда следует, что его полугруппа марковская. Наша цель показать, что $\exp \left(-t \mathfrak{T}^{\alpha}\right)$ является изотропной марковской полугруппой на ультраметрическом пространстве с мерой $\left(\mathbb{Q}_{p}^{n}, d_{p}, \mu_{p}^{n}\right)$.

Наше первое наблюдение состоит в том, что спектр симметричного оператоpa $\left(\mathfrak{T}^{\alpha}, \mathscr{V}_{c}\right)$ совпадает с областью значений функции $\zeta \mapsto\|\zeta\|_{p}^{\alpha}$ :

$$
\operatorname{spec} \mathfrak{T}^{\alpha}=\left\{p^{k \alpha}: k \in \mathbb{Z}\right\} \cup\{0\} .
$$

Собственное пространство $\mathscr{H}(\lambda)$ оператора $\left(\mathfrak{T}^{\alpha}, \mathscr{V}_{c}\right)$, соответствующее собственному значению $\lambda=p^{k \alpha}$, порождено функцией

$$
f_{k}=\frac{1}{\mu_{p}^{n}\left(p^{k} \mathbb{Z}_{p}^{n}\right)} \mathbf{1}_{p^{k} \mathbb{Z}_{p}^{n}}-\frac{1}{\mu_{p}^{n}\left(p^{k-1} \mathbb{Z}_{p}^{n}\right)} \mathbf{1}_{p^{k-1} \mathbb{Z}_{p}^{n}}
$$

и всеми ее сдвигами $f_{k}(\cdot+a)$ при $a \in \mathbb{Q}_{p}^{n} / p^{k} \mathbb{Z}_{p}^{n}$. Действительно, вычисляя преобразование Фурье функции $f_{k}$ :

$$
\widehat{f}_{k}(\zeta)=\mathbf{1}_{\left\{\|\zeta\|_{p} \leqslant p^{k}\right\}}-\mathbf{1}_{\left\{\|\zeta\|_{p} \leqslant p^{k-1}\right\}}=\mathbf{1}_{\left\{\|\zeta\|_{p}=p^{k}\right\}},
$$

получаем

$$
\widehat{\mathfrak{T}^{\alpha} f_{k}}(\zeta)=\|\zeta\|_{p}^{\alpha} \widehat{f_{k}}(\zeta)=p^{k \alpha} \widehat{f}_{k}(\zeta)
$$


Все вышеизложенное показывает, что оператор $\mathfrak{T}^{\alpha}$ совпадает с изотропным лапласианом $\mathscr{L}_{\alpha}$ на $\left(\mathbb{Q}_{p}^{n}, d_{p}, \mu_{p}^{n}\right)$, связанным с функцией распределения расстояний

$$
\sigma_{\alpha}(r)=\exp \left(-\left(\frac{p}{r}\right)^{\alpha}\right)
$$

а полугруппа $\exp \left(-t \mathfrak{T}^{\alpha}\right)$ совпадает с изотропной полугруппой $\left\{P_{\alpha}^{t}\right\}$.

Заметим, что соответствующая внутренняя ультраметрика есть

$$
d_{p *}(x, y)=\left(\frac{\|x-y\|_{p}}{p}\right)^{\alpha} .
$$

Спектральная функция $N_{\alpha}(x, \tau)=N_{\alpha}(\tau)$ является неубывающей непрерывной слева ступенчатой функцией, имеющей скачки в точках $\tau_{k}=p^{k \alpha}, k \in \mathbb{Z}$, и принимающей в этих точках значения $N_{\alpha}\left(\tau_{k}\right)=p^{(k-1) n}$. Следовательно,

$$
N_{\alpha}(\tau) \simeq \tau^{n / \alpha}
$$

В частности, функция $\tau \mapsto N_{\alpha}(\tau)$ удовлетворяет свойству удвоения, и из теоремы 2.14 вытекает следующий результат.

ТеОрема 5.17. Полугруппа $\exp \left(-t \mathfrak{T}^{\alpha}\right)$ на $\mathbb{Q}_{p}^{n}$ допускает непрерывное ядро теплопроводности $p_{\alpha}(t, x, y)$, удовлетворяющее оценке

$$
p_{\alpha}(t, x, y) \simeq \frac{t}{\left(t^{1 / \alpha}+\|x-y\|_{p}\right)^{n+\alpha}} .
$$

$B$ частности, полугруппа $\exp \left(-t \mathfrak{T}^{\alpha}\right)$ является невозвратной тогда и толъко тогда, когда $\alpha<n$. В невозвратном случае функиия Грина (= риссово ядро Тайблесона) удовлетворяет тождеству

$$
g_{\alpha}(x, y)=\frac{1-p^{-\alpha}}{1-p^{\alpha-n}} \frac{1}{\|x-y\|_{p}^{n-\alpha}} .
$$

Заметим, что верхняя оценка в (5.27) была доказана в [50].

Определение 5.7 инвариантного относительно вращений лапласиана на $\mathbb{Q}_{p}$ можно перенести на $\mathbb{Q}_{p}^{n}$. Оператор Тайблесона $\mathfrak{T}^{\alpha}$ является примером инвариантного относительно вращений лапласиана. Теорема 5.8 , следствия 5.9 и 5.10 и их доказательства остаются справедливыми также для $\mathbb{Q}_{p}^{n}$. Здесь мы дадим короткое доказательство немного более слабого результата, важного для нас. Положим $\mathfrak{T}=\mathfrak{T}^{1}$.

Теорема 5.18. Равенство $\left(\mathscr{L}, \mathscr{V}_{c}\right)=\left(\psi(\mathfrak{T}), \mathscr{V}_{c}\right)$, где $\psi-$ произвольная возрастающая биекция $[0, \infty) \rightarrow[0, \infty)$, дает полное описание класса изотропньх лапласианов на ультраметрическом пространстве с мерой $\left(\mathbb{Q}_{p}^{n}, d_{p}, \mu_{p}^{n}\right)$.

ДокАЗАтельство. Пусть $\psi:[0, \infty) \mapsto[0, \infty)$ - возрастающая биекция. По теореме 3.1 оператор $\left(\psi(\mathfrak{T}), \mathscr{V}_{c}\right)$ является изотропным лапласианом.

Обратно, пусть $\left(\mathscr{L}, \mathscr{V}_{c}\right)$ - изотропный лапласиан на $\left(\mathbb{Q}_{p}^{n}, d_{p}, \mu_{p}^{n}\right)$. Пусть $d_{p *}-$ внутреннее расстояние, связанное с $\mathscr{L}$. По построению $d_{p *}$ является возрастающей функцией от $d_{p}$ (см. (2.14)). Поскольку область значений $d_{p}$ есть множество $\left\{p^{k}: k \in \mathbb{Z}\right\} \cup\{0\}$, можно выбрать возрастающую биекцию $\varphi:[0, \infty) \rightarrow$ $[0, \infty)$ такую, что $d_{p *}=\phi\left(d_{p}\right)$. Пусть $\lambda(B)$ и $\tau(B)-$ собственные значения 
соответственно операторов $\left(\mathscr{L}, \mathscr{V}_{c}\right)$ и $\left(\mathfrak{T}, \mathscr{V}_{c}\right)$, соответствующие шару $B \subset \mathbb{Q}_{p}^{n}$. Поскольку внутреннее расстояние, связанное с $\mathfrak{T}$, есть $p^{-1} d_{p}$, получаем

$$
\begin{aligned}
\lambda(B) & =\frac{1}{\operatorname{diam}_{p *}(B)}=\frac{1}{\varphi\left(\operatorname{diam}_{p}(B)\right)} \\
& =\frac{1}{\varphi(p / \tau(B))}=: \psi(\tau(B)),
\end{aligned}
$$

где $\psi(s)=1 / \varphi(p / s)$ - возрастающая биекция множества $[0, \infty)$ на себя.

Поскольку $\left(\mathscr{L}, \mathscr{V}_{c}\right)$ и $\left(\psi(\mathfrak{T}), \mathscr{V}_{c}\right)$ - изотропные лапласианы, определенные на ультраметрическом пространстве с мерой $\left(\mathbb{Q}_{p}^{n}, d_{p}, \mu_{p}^{n}\right)$, у которых множества собственных значений совпадают, мы получаем

$$
\left(\mathscr{L}, \mathscr{V}_{c}\right)=\left(\psi(\mathfrak{T}), \mathscr{V}_{c}\right)
$$

или, равносильно, в терминах преобразования Фурье,

$$
\widehat{\mathscr{L} f}(\zeta)=\psi\left(\|\zeta\|_{p}\right) \widehat{f}(\zeta)
$$

для всех $f \in \mathscr{V}_{c}$ и $\zeta \in \mathbb{Q}_{p}^{n}$, что завершает доказательство теоремы.

\section{6. Случайные блуждания на дереве и процессы со скачками на его границе}

6.1. Корневые деревья и их границы. Дерево - это связный граф $T$ без циклов (замкнутых путей длины $\geqslant 3$ ). По умолчанию мы отождествляем $T$ с его множеством вершин, которое предполагается бесконечным. Мы пишем $u \sim v$, если $u, v \in T$ являются соседями. Для любой пары вершин $u, v \in T$ имеется единственный кратчайший путь

$$
\pi(u, v)=\left[u=v_{0}, v_{1}, \ldots, v_{k}=v\right],
$$

называемый геодезическим отрезком, такой, что $v_{i-1} \sim v_{i}$ и все $v_{i}$ различны. Если $u=v$, то это пустой, или тривиальный, путь. Число $k-$ длина пути (расстояние на графе между $u$ и $v$ ). В дереве $T$ выберем и зафиксируем корневую вериину о. Мы обозначаем через $|v|$ длину $\pi(o, v)$. Выбор корня индуцирует частичный порядок на $T$, где $u \leqslant v$, когда $u \in \pi(o, v)$. Каждая вершина $v \in T \backslash\{o\}$ имеет единственного предшественника $v^{-}$относительно $o$, который является единственным соседом вершины $v$ на $\pi(o, v)$. Таким образом, множество всех (неориентированных) ребер графа $T$ есть

$$
E(T)=\left\{\left[v^{-}, v\right]: v \in T, v \neq o\right\} .
$$

Для $u \in T$ элементы множества

$$
\left\{v \in T: v^{-}=u\right\}
$$

являются последователями вершины $u$, а его мощность $\operatorname{deg}^{+}(u)$ есть исходящая степень вершины $u$. 
В этом и следующем разделах мы считаем, что

$$
2 \leqslant \operatorname{deg}^{+}(u)<\infty \quad \text { для каждой вершины } u \in T \text {. }
$$

(Геодезический) луч в $T$ - это односторонний бесконечный путь $\pi=\left[v_{0}, v_{1}\right.$, $\left.v_{2}, \ldots\right]$ такой, что $v_{n-1} \sim v_{n}$ и все $v_{n}$ различны. Два луча эквивалентны, если их симметрическая разность (как множеств вершин) конечна. Конеи, дерева $T$ - это класс эквивалентности лучей. Обычно мы будем использовать символы $x, y, z$ для обозначения концов (и символы $u, v, w$ для обозначения вершин). Множество всех концов дерева $T$ обозначается через $\partial T$. Это граница дерева на бесконечности. Для любых $u \in T$ и $x \in \partial T$ имеется единственный луч $\pi(u, x)$, представляющий конец $x$ и начинающийся в $u$. Мы обозначим

$$
\widehat{T}=T \cup \partial T \text {. }
$$

Для $u \in T$ ветвъ дерева $T$ с корнем в $u$ - это поддерево $T_{u}$, которое мы отождествляем с множеством его вершин

$$
T_{u}=\{v \in T: u \leqslant v\}
$$

так что $T_{o}=T$. Мы используем обозначение $\partial T_{u}$ для множества всех концов дерева $T$, которые обладают представляющим путем, содержащимся в $T_{u}$, а также обозначение $\widehat{T}_{u}=T_{u} \cup \partial T_{u}$.

Для $w, z \in \widehat{T}$ определим их конфлюэнт $w \wedge z=w \wedge_{o} z$ относительно корня $o$ соотношением

$$
\pi(o, w \wedge z)=\pi(o, w) \cap \pi(o, z) .
$$

Это последний общий элемент на геодезических $\pi(o, w)$ и $\pi(o, z)$; он является вершиной дерева $T$, если только не выполняется $w=z \in \partial T$; см. рис. 4.

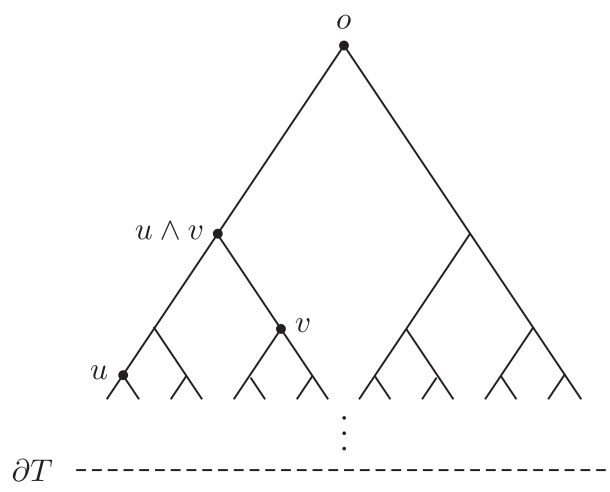

Рис. 4

Один из наиболее распространенных способов определения ультраметрики на $\widehat{T}$ таков:

$$
d_{e}(z, w)= \begin{cases}0, & \text { если } z=w, \\ e^{-|z \wedge w|}, & \text { если } z \neq w .\end{cases}
$$


Тогда $\widehat{T}$ компактно, а $T$ открыто и всюду плотно. Нас в основном интересует компактное ультраметрическое пространство $\partial T$. В метрике $d_{e}$ из (6.3) каждый $d_{e}$-шар с центром $x \in \partial T$ имеет вид $\partial T_{u}$ для некоторого $u \in \pi(o, x)$. Действительно,

$$
\partial T_{u}=B_{e^{-|u|}}(x) \quad \text { для каждой } u \in \pi(o, x)
$$

и

$$
\Lambda_{d_{e}}(x)=\left\{e^{-|u|}: u \in \pi(o, x)\right\} .
$$

Обратно, мы можем начать с компактного ультраметрического пространства $(X, d)$, не имеющего изолированных точек, и построить дерево $T$ следующим образом: множество вершин $T$ - это множество

$$
\mathscr{B}=\left\{B_{r}(x): x \in X, r>0\right\}
$$

всех замкнутых шаров в $(X, d)$, которое уже встречалось в разделе 3. Здесь можно (при желании) считать, что $r \in \Lambda_{d}(x)$.

Теперь рассмотрим любой шар $v=B \in \mathscr{B}$ как вершину дерева $T$. Выберем нашу корневую вершину как $o=X$; она принадлежит $\mathscr{B}$ в силу компактности. Окрестность задается отношением предшественников шаров, как в определении 3.6. То есть, если $v=B$, то $u=B^{\prime}-$ предшественник $v^{-}$вершины $v$ в дереве $T$. В силу компактности каждый $x$ имеет только конечное число последователей, а так как в $X$ нет изолированных точек, у каждой вершины есть хотя бы два последователя, так что выполняется соотношение (6.1).

Это определяет структуру дерева. Для любого $x \in X$ множество всех шаров $B_{r}(x), r \in \Lambda_{d}(x)$, упорядоченное по убыванию, образует множество вершин луча в $T$, начинающегося в $о$. В качестве несложного упражнения можно показать, что отображение, ставящее в соответствие точке $x$ конец дерева $T$, представленный этим лучом, является гомеоморфизмом из $X$ на $\partial T$. Таким образом, можно отождествить $X$ и $\partial T$ как ультраметрические пространства.

При этом отождествлении, если первоначально вершина $u$ интерпретировалась как шар $B_{r}(x), r \in \Lambda_{d}(x)$, то множество $\partial T_{u}$ концов ветви $T_{u}$ просто совпадает с шаром $B_{r}(x)$. Таким образом, мы отождествляем каждую вершину $u$ дерева $T$ с множеством $\partial T_{u}$.

Если начать с произвольного локально конечного дерева и рассмотреть его пространство концов как ультраметрическое пространство $X$, то вышеприведенная конструкция не восстанавливает вершины с исходящей степенью 1, так что в общем случае мы не получим обратно то дерево, с которого начали. Однако вышеприведенная конструкция устанавливает биективное соответствие между компактными ультраметрическими пространствами без изолированных точек (совершенными ультраметрическими пространствами) и локально конечными корневыми деревьями с исходящими степенями $\geqslant 2$ (ср. с [32]).

Хорошо известно, что любое ультраметрическое пространство $X$, которое и компактно, и совершенно, гомеоморфно тернарному канторову множеству $C \subset[0,1]$. Когда $X$ не компактно, но все еще совершенно, имеется гомеоморфизм $X \simeq C \backslash\{p\}$, где $p \in C$ - любая фиксированная точка. 
Далее до конца п. 6.2 мы отказываемся от обозначения $X$ для компактного и совершенного ультраметрического пространства.

Мы рассматриваем $X$ как граничу дТ локально конечного корневого дерева с исходящей степенъю $\geqslant 2$.

В конце мы обсудим, как можно работать при наличии вершин с исходящей степенью 1, а также в некомпактном случае.

Есть много способов, как снабдить $\partial Т$ ультраметрикой, которая будет определять ту же самую топологию и те же компактные-открытые шары $\partial T_{x}, x \in T$ (возможно, с другими радиусами), что и стандартная метрика (6.3). Следующее определение является своего рода ультраметрическим аналогом элемента длины.

ОПРЕДЕЛЕниЕ 6.1. Пусть $T$ - локально конечное корневое дерево, в котором $\operatorname{deg}^{+}(x) \geqslant 2$ для всех $x$. Улътраметрический элемент - это функция $\phi: T \rightarrow(0, \infty)$, удовлетворяющая следующим условиям:

(i) $\phi\left(v^{-}\right)>\phi(v)$ для каждой $v \in T \backslash\{o\}$;

(ii) $\lim \phi\left(v_{n}\right)=0$ вдоль каждого геодезического луча $\pi=\left[v_{0}, v_{1}, v_{2}, \ldots\right]$. Такая функция индуцирует ультраметрику $d_{\phi}$ на $\partial T$, задаваемую формулой

$$
d_{\phi}(x, y)= \begin{cases}0, & \text { если } x=y \\ \phi(x \wedge y), & \text { если } x \neq y\end{cases}
$$

Шары в этой ультраметрике снова суть множества

$$
\partial T_{u}=B_{\phi(u)}(x), \quad x \in \partial T_{u} .
$$

Заметим, что условие (ii) в определении необходимо для того, чтобы каждый конец дерева $T$ был неизолированным в метрике $d_{\phi}$. Метрика $d_{e}$ из (6.3), конечно, индуцирована ультраметрическим элементом $\phi(x)=e^{-|x|}$.

Лемма 6.2. Для дерева как в определении 6.1 каждая ультраметрика на $\partial T$, чъи замкнутые шары являются множествами $\partial T_{u}, u \in T$, индуиирована ультраметрическим элементом на $T$.

ДокАЗАТЕЛьство. Для ультраметрики $d$, удовлетворяющей условию леммы, положим $\phi(v)=\operatorname{diam}\left(\partial T_{v}\right)$, где диаметр берется относительно метрики $d$. Поскольку $\operatorname{deg}^{+}\left(v^{-}\right) \geqslant 2$ для любого $v \in T \backslash\{o\}$, шар $\partial T_{v^{-}}$является объединением по крайней мере двух непересекающихся шаров $\partial T_{u}$ с $u^{-}=v^{-}$. Поэтому должно выполняться неравенство $\operatorname{diam}\left(\partial T_{v}\right)<\operatorname{diam}\left(\partial T_{v^{-}}\right)$, и свойство (i) имеет место. Поскольку ни один конец не изолирован, $\phi$ удовлетворяет свойству (ii). Теперь ясно, что $d_{\phi}=d$. Лемма доказана.

Ввиду указанного соответствия между ультраметрикой и ультраметрическим элементом, всюду ниже мы заменяем в обозначениях индекс $d$, указывающий на метрику $d=d_{\phi}$, индексом $\phi$, указывающим на ультраметрический элемент. Заметим, что

$$
\operatorname{diam}_{\phi}(\partial T)=\phi(o), \quad \Lambda_{\phi}(x)=\{\phi(u): u \in \pi(o, x)\}, \quad \Lambda_{\phi}=\{\phi(v): v \in T\} .
$$


Также заметим, что для любых $x \in \partial T$ и $v \in \pi(o, x)$ шары в метрике $d_{\phi}$ удовлетворяют тождеству

$$
B_{r}(x)=B_{r}^{\phi}(x)= \begin{cases}\partial T_{v} & \text { для } \phi(v) \leqslant r<\phi\left(v^{-}\right), \text {если } v \neq o ; \\ \partial T & \text { для } r \geqslant \phi(o), \text { если } v=o .\end{cases}
$$

6.2. Изотропные процессы со скачками на границе дерева. Таким образом, можно рассматривать изотропные процессы, определенные формулами (1.3)-(1.8), на пространстве $X=\partial T$. Поскольку это пространство компактно, можно считать, что эталонная мера $\mu$ является вероятностной мерой на $\partial T$. Выбрав меру $\mu$ на $\partial T$, функцию распределения расстояний $\sigma$ со свойствами (1.5) и ультраметрический элемент $\phi$ на $T$, получим $\left(d_{\phi}, \mu, \sigma\right)$-процесс на $\partial T$, который будем также называть $(\phi, \mu, \sigma)$-процессом на $\partial T$. Мы можем подробно выписать полугруппу и ее переходные вероятности. Именно, для $x \in \partial T$ и $\pi(0, x)=\left[o=v_{0}, v_{1}, v_{2}, \ldots\right]$, используя $(6.5)$, получаем

$$
P^{t} f(x)=\sum_{n=0}^{\infty} c_{n}^{t} \mathrm{Q}_{\phi\left(v_{n}\right)} f(x)
$$

где

$$
c_{0}^{t}=1-\sigma^{t}\left(\phi\left(v_{0}\right)\right), \quad c_{n}^{t}=\sigma^{t}\left(\phi\left(v_{n-1}\right)\right)-\sigma^{t}\left(\phi\left(v_{n}\right)\right), \quad n \geqslant 1 .
$$

Таким образом, для произвольных $u \in T$ и $x \in \partial T$, как и выше, имеем

$$
\mathrm{P}\left[X_{t} \in \partial T_{u} \mid X_{0}=x\right]=\sum_{n=0}^{\infty} c_{n}^{t} \frac{\mu\left(\partial T_{v_{n}} \cap \partial T_{u}\right)}{\mu\left(\partial T_{v_{n}}\right)} .
$$

Стандартный $(d, \mu)$-процесс в смысле определения 2.9 в случае метрики $d=d_{\phi}$ будет теперь именоваться стандартным $(\phi, \mu)$-процессом.

6.3. Простые случайные блуждания на дереве. На деревьях имеется хорошо изученный класс стохастических процессов, а именно случайные блуждания. Наша цель - проанализировать, как случайные блуждания на дереве связаны с изотропными процессами на границе дерева. Значительная часть приводимого ниже материала взята из книги В. Вёсса [63]. Более старая рекомендуемая ссылка - это основополагающая работа П. Картье [12].

Простое случайное блуждание на локально конечном бесконечном дереве $T$ индуцируется его стохастической переходной матрицей $\mathscr{P}=(p(u, v))_{u, v \in T}$, обладающей тем свойством, что $p(u, v)>0$ тогда и только тогда, когда $u \sim v$. Возникающая марковская цепь с дискретным временем (случайное блуждание) обозначается через $\left(Z_{n}\right)_{n \geqslant 0}$. Его $n$-шаговые переходные вероятности

$$
p^{(n)}(u, v)=\mathrm{P}_{u}\left[Z_{n}=v\right], \quad u, v \in T,
$$

являются элементами $n$-й степени матрицы $\mathscr{P}$. Здесь $\mathrm{P}_{u}$ обозначает вероятностную меру на пространстве траекторий, начинающихся в $u$. Мы предполагаем, что случайное блуждание является невозвратным, т. е. с вероятностью 1 
оно посещает любое конечное множество только конечное число раз. Таким образом, $0<G(u, v)<\infty$ для всех $u, v \in T$, где

$$
G(u, v)=\sum_{n=0}^{\infty} p^{(n)}(u, v)
$$

- функция Грина случайного блуждания. Кроме того, мы также будем существенно использовать величины

$$
\begin{aligned}
& F(u, v)=\mathrm{P}_{u}\left[Z_{n}=v \text { для некоторого } n \geqslant 0\right], \\
& U(v, v)=\mathrm{P}_{v}\left[Z_{n}=v \text { для некоторого } n \geqslant 1\right] .
\end{aligned}
$$

Нам потребуется ряд связывающих их тождеств, и мы начнем с нескольких, справедливых для всех $u, v \in T$ :

$$
\begin{aligned}
G(u, v) & =F(u, v) G(v, v) ; \\
G(v, v) & =\frac{1}{1-U(v, v)} ; \\
U(v, v) & =\sum_{u} p(v, u) F(u, v) ; \\
F(u, v) & =F(u, w) F(w, v), \quad \text { как только } w \in \pi(u, v) .
\end{aligned}
$$

Первые три тождества выполняются для произвольных счетных марковских цепей, в то время как (6.10) справедливо для деревьев. Эти тождества показывают, что величины $G, U, F$ полностью определяются уже значениями $F(u, v)$ для $u \sim v$. Ниже будут приводиться и использоваться также и другие тождества из [63; гл. 9].

В силу невозвратности случайное блуждание $Z_{n}$ должно сходиться к случайному концу (см., например, [12] или [63; теорема 9.18]).

Лемма 6.3. Существует ӘТ-значная случайная величина $Z_{\infty}$ такая, что для каждой начальной точки $u \in T$

$$
\mathrm{P}_{u}\left[Z_{n} \rightarrow Z_{\infty} \text { в топологии } \widehat{T}\right]=1 .
$$

Вкратце рассуждение таково: в силу невозвратности, траектории случайного блуждания должны почти наверное сгущаться на $\partial T$. Если бы такая траектория имела две различные точки сгущения, скажем, $x$ и $y$, то, поскольку случайное блуждание является простым, траектория посещала бы вершину $x \wedge_{u} y$ бесконечно много раз, что может случиться только с вероятностью 0.

Для любой вершины $u \in T$ рассмотрим пределъное распределение $\nu_{u}$, которое является борелевской мерой на $\partial T$, определенной для любого борелевского множества $B \subset \partial T$ следующим образом:

$$
\nu_{u}(B)=\mathrm{P}_{u}\left[Z_{\infty} \in B\right] .
$$

Множества $\partial T_{u}, u \in T$ (в совокупности с пустым множеством) образуют полуалгебру, которая порождает борелевскую $\sigma$-алгебру на пространстве $\partial T$. Таким 
образом, мера $\nu_{u}$ определяется своими значениями на указанных множествах. Имеется явная формула (ср. с [12] или [63; предложение 9.23]), справедливая при $v \neq o$ :

$$
\nu_{u}\left(\partial T_{v}\right)= \begin{cases}F(u, v) \frac{1-F\left(v, v^{-}\right)}{1-F\left(v^{-}, v\right) F\left(v, v^{-}\right)}, & \text {если } u \in\{v\} \cup\left(T \backslash T_{v}\right), \\ 1-F(u, v) \frac{F\left(v, v^{-}\right)-F\left(v^{-}, v\right) F\left(v, v^{-}\right)}{1-F\left(v^{-}, \nu\right) F\left(v, v^{-}\right)}, & \text {если } u \in T_{v} .\end{cases}
$$

Гармоническая функиия - это функция $h: T \rightarrow \mathbb{R}$, удовлетворяющая равенству $\mathscr{P} h=h$, где

$$
\operatorname{Ph}(u)=\sum_{v} p(u, v) h(v) .
$$

Для любого борелевского множества $B \subset \partial T$ функция $u \mapsto \nu_{u}(B)$ является ограниченной гармонической функцией. Можно доказать, что все $\nu_{u}$ сравнимы в следующем смысле: $p^{(k)}(u, v) \nu_{u} \leqslant \nu_{v}$, где $k$ - длина $\pi(u, v)$. Итак, для любой функции $\varphi \in L^{1}\left(\partial T, \nu_{o}\right)$ функция $h_{\varphi}$, определенная равенством

$$
h_{\varphi}(u)=\int_{\partial T} \varphi d \nu_{u}
$$

является конечной и гармонической на $T$. Ее часто называют преобразованием Пуассона функции $\varphi$.

Далее, определим меру m на $T$ следующим образом:

$$
\mathrm{m}(o)=1,
$$

а для $v \in T \backslash\{o\}$ таких, что $\pi(o, v)=\left[o=v_{0}, v_{1}, \ldots, v_{k}=v\right]$, полагаем

$$
\mathrm{m}(v)=\frac{p\left(v_{0}, v_{1}\right) p\left(v_{1}, v_{2}\right) \cdots p\left(v_{k-1}, v_{k}\right)}{p\left(v_{1}, v_{0}\right) p\left(v_{2}, v_{1}\right) \cdots p\left(v_{k}, v_{k-1}\right)} .
$$

Тогда для всех $u, v \in T$ имеем

$$
\mathrm{m}(u) p(u, v)=\mathrm{m}(v) p(v, u) \quad \text { и, следовательно, } \quad \mathrm{m}(u) G(u, v)=\mathrm{m}(v) G(v, u) ;
$$

т. е. случайное блуждание обратимо. Это позволяет нам использовать интерпретацию $(T, \mathscr{P}, \mathrm{m})$ как электрической цепи: см., например, работу М. Ямасаки [65], книгу П. М. Соарди [54] или - с обозначениями, используемыми в настоящей работе, - книгу [63; гл. 4]. Каждое ребро $e=\left[v^{-}, v\right] \in E(T)$ рассматривается как электрический проводник с проводимостъю

$$
a\left(v^{-}, v\right)=\mathrm{m}(v) p\left(v, v^{-}\right) .
$$

Форма Дирихле $\mathscr{E}_{T}=\mathscr{E}_{T, \mathscr{P}}$ для функций $f, g: T \rightarrow \mathbb{R}$ определяется формулой

$$
\mathscr{E}_{T}(f, g)=\sum_{\left[v^{-}, v\right] \in E(T)}\left(f(v)-f\left(v^{-}\right)\right)\left(g(v)-g\left(v^{-}\right)\right) a\left(v^{-}, v\right) .
$$

Областью определения этой формы Дирихле является пространство

$$
\mathscr{D}(T)=\mathscr{D}(T, \mathscr{P})=\left\{f: T \rightarrow \mathbb{R} \mid \mathscr{E}_{T}(f, f)<\infty\right\} .
$$


6.4. Гармонические функции конечной энергии и их граничные значения. Нас интересует подпространство

$$
\mathscr{H} \mathscr{D}(T)=\mathscr{H} \mathscr{D}(T, \mathscr{P})=\{h \in \mathscr{D}(T, \mathscr{P}): \mathscr{P} h=h\}
$$

гармонических функций с конечной энергией. Терминология пришла из интерпретации таких функций как потенциалов электрического тока и того, что $\mathscr{E}_{T}(h, h)$ - энергия тока.

Каждая функция в $\mathscr{H} \mathscr{D}(T, \mathscr{P})$ является преобразованием Пуассона некоторой функции $\varphi \in L^{2}\left(\partial T, \nu_{o}\right)$. Это справедливо не только для деревьев, но и для обратимых конечнозначных марковских цепей общего вида, и вытекает из следующих фактов.

(1) Каждая функция из $\mathscr{H} \mathscr{D}$ является разностью двух неотрицательных функций из $\mathscr{H} \mathscr{D}$.

(2) Каждую неотрицательную функцию из $\mathscr{H} \mathscr{D}$ можно аппроксимировать монотонно возрастающей последовательностью неотрицательных ограниченных функций из $\mathscr{H} \mathscr{D}$.

(3) Каждая ограниченная гармоническая функция является преобразованием Пуассона ограниченной функции на границе $\partial T$.

Граница $\partial T$ является (активной частью) границы Мартина, причем $\nu_{u}-$ предельное на $\partial T$ распределение марковской цепи, начинающейся в $u$. Факты (1) и (2) содержатся в [65] и [54], в то время как (3) является частью общей теории границ Мартина (см., например, [63; теорема 7.61]).

Итак, мы определяем форму $\mathscr{E} \mathscr{H} \mathscr{D}$ на $\partial T$, полагая

$$
\begin{aligned}
\mathscr{D}(\partial T, \mathscr{P}) & =\left\{\varphi \in L^{1}\left(\partial T, \nu_{o}\right): \mathscr{E}_{T}\left(h_{\varphi}, h_{\varphi}\right)<\infty\right\}, \\
\mathscr{E}_{\mathscr{H} \mathscr{D}}(\varphi, \psi) & =\mathscr{E}_{T}\left(h_{\varphi}, h_{\psi}\right) \quad \text { для } \varphi, \psi \in \mathscr{D}(\partial T, \mathscr{P}) .
\end{aligned}
$$

6.5. Процессы со скачками на границе дерева. Прилагая значительные усилия, Дж. Кигами [36] выводит выражение для формы $\mathscr{E} \mathscr{H} \mathscr{D}(\varphi, \psi)$, определенной в (6.16), устанавливает ее свойства регулярности и затем изучает процесс со скачками на $\partial T$, индуцированный этой формой Дирихле. Мы называем этот процесс граничным прочессом, связанным со случайным блужданием на $T$.

Покажем, что для $\mathscr{E} \mathscr{H} \mathscr{D}$ имеется довольно простое интегральное представление. Определим ядро Наим на $\partial T \times \partial T$ формулой

$$
\Theta_{o}(x, y)= \begin{cases}\frac{\mathrm{m}(o)}{G(o, o) F(o, x \wedge y) F(x \wedge y, o)}, & \text { если } x \neq y, \\ +\infty, & \text { если } x=y .\end{cases}
$$

В нашем случае имеем $\mathrm{m}(o)=1$, но из соображений общности мы пока не используем эту нормировку меры.

ТЕОРЕма 6.4. Для любого простого невозвратного случайного блуждания на дереве $T$ с корнем о и для всех функиий $\varphi, \psi$ в $\mathscr{D}(\partial T, \mathscr{P})$ имеет место представление

$$
\mathscr{E}_{\mathscr{H} \mathscr{D}}(\varphi, \psi)=\frac{1}{2} \int_{\partial T} \int_{\partial T}(\varphi(x)-\varphi(y))(\psi(x)-\psi(y)) \Theta_{o}(x, y) d \nu_{o}(x) d \nu_{o}(y) .
$$


Доказательство теоремы 6.4 дано в [20] в рамках теории потенциала на пространствах Грина, которые локально евклидовы. Определение ядра Наим дается в [44] в той же общности. Однако деревья, даже когда они рассматриваются как метрические графы, не являются локально евклидовыми. В этом смысле, до сих пор определение ядра Наим и доказательство теоремы 6.4 в устраивающей нас общности были недостаточно отражены в литературе. "Недостающие звенья" будут даны в полной общности в готовящейся к печати работе А. Георгакопулоса (A. Georgakopoulos) и В. А. Каймановича (V. A. Kaimanovich).

Здесь мы даем прямое и простое доказательство теоремы 6.4 для специального случая деревьев. Начнем со следующего наблюдения.

Лемма 6.5. Мера $\Theta_{o}(x, y) d \nu_{o}(x) d \nu_{o}(y)$ на $\partial T \times \partial T$ инвариантна относительно смены базовой точки (корня) о.

ДокАЗАТЕЛЬСтво. Мы хотим заменить базовую точку о некоторой другой $u \in T$. Можно считать, что $u \sim o$. Действительно, тогда можно шаг за шагом заменить текущую базовую точку одной из соседних с ней, чтобы получить результат для произвольной $u$.

Напомним, что конфлюэнт, который появился в определении (6.17) ядра $\Theta_{o}$, зависит от корня $о$, в то время как для $\Theta_{x}$ он становится конфлюэнтом относительно $x$ как нового корня. Хорошо известный факт состоит в том, что

$$
\frac{d \nu_{u}}{d \nu_{o}}(x)=K(u, x):=\frac{G\left(u, u \wedge_{o} x\right)}{G\left(o, u \wedge_{o} x\right)},
$$

где $K$ называется ядром Мартина. Итак, нам нужно показать, что для всех $x, y \in \partial T(x \neq y)$ выполняется равенство

$$
\frac{\mathrm{m}(o)}{G(o, o) F\left(o, x \wedge_{o} y\right) F\left(x \wedge_{o} y, o\right)}=\frac{\mathrm{m}(u) K(u, x) K(u, y)}{G(u, u) F\left(u, x \wedge_{u} y\right) F\left(x \wedge_{u} y, u\right)} .
$$

Рассмотрим четыре случая.

Случай 1: $x, y \in \partial T_{u}$. Тогда $x \wedge_{o} y=x \wedge_{u} y=: v \in T_{u}$ и $u \wedge_{o} x=u \wedge_{o} y=u$. Таким образом, используя $(6.7),(6.10)$ и тот факт, что в силу $(6.13)$

$$
\frac{\mathrm{m}(u)}{G(o, u)}=\frac{\mathrm{m}(o)}{G(u, o)},
$$

получаем

$$
\begin{aligned}
\frac{\mathrm{m}(u) K(u, x) K(u, y)}{G(u, u) F\left(u, x \wedge_{u} y\right) F\left(x \wedge_{u} y, u\right)} & =\frac{\mathrm{m}(u)}{G(u, u) F(u, v) F(v, u)}\left(\frac{G(u, u)}{G(o, u)}\right)^{2} \\
& =\frac{\mathrm{m}(o) G(u, u)}{F(u, v) F(v, u) G(o, u) G(u, o)} \\
& =\frac{\mathrm{m}(o)}{F(u, v) F(v, u) F(o, u) F(u, o) G(o, o)} \\
& =\frac{\mathrm{m}(o)}{F(o, v) F(v, o) G(o, o)},
\end{aligned}
$$

что и требовалось доказать. 
Случай 2: $x, y \in \partial T \backslash \partial T_{u}$. Тогда

$$
x \wedge_{o} y=x \wedge_{u} y=: w \in T \backslash T_{u}, \quad u \wedge_{o} x=u \wedge_{o} y=o .
$$

Случай 3: $x \in \partial T_{u}, y \in \partial T \backslash \partial T_{u}$. Тогда

$$
x \wedge_{o} y=o, \quad x \wedge_{u} y=u, \quad u \wedge_{o} x=u, \quad u \wedge_{o} y=o .
$$

Случай 4: $x \in \partial T \backslash \partial T_{u}, y \in \partial T_{u}$. Этот случай аналогичен случаю 3 ( $x$ и $y$ меняются ролями).

Во всех случаях 2-4 доказательство проводится аналогично случаю 1. Лемма доказана.

Для доказательства теоремы 6.4 нам нужно еще несколько фактов, связанных с сетевой постановкой; ср., например, с [63; § 4.D].

Пространство $\mathscr{D}(T)$ из (6.15), будучи оснащенным скалярным произведением

$$
(f, g)=\mathscr{E}_{T}(f, g)+f(o) g(o)
$$

является гильбертовым пространством. Подпространство $\mathscr{D}_{0}(T)$ определяется как замыкание пространства функций с конечными носителями в $\mathscr{D}(T)$. Оно является собственным подпространством тогда и только тогда, когда случайное блуждание невозвратно, и в этом случае функция $G_{v}(u)=G(u, v)$ лежит в $\mathscr{D}_{0}(T)$ для любого $v \in T$ (см. [65], [54]). Нам потребуется формула

$$
\mathscr{E}_{T}\left(f, G_{v}\right)=\mathrm{m}(v) f(v) \quad \text { для каждого } f \in \mathscr{D}_{0}(T) .
$$

Ветвь $T_{w}$ дерева $T$ (для $\left.w \in T \backslash\{o\}\right)$ можно рассматривать как подсеть, оснащенную теми же проводимостями $a(u, v)$ для $[u, v] \in E\left(T_{w}\right)$. Соответствующая мера на $T_{w}$ есть

$$
\mathrm{m}_{T_{w}}(u)=\sum_{v \in T_{z}: v \sim u} a(u, v)= \begin{cases}\mathrm{m}(u), & \text { если } u \in T_{w} \backslash\{w\}, \\ \mathrm{m}(w)-a\left(w, w^{-}\right), & \text {если } u=w .\end{cases}
$$

Получающееся случайное блуждание на $T_{w}$ имеет переходные вероятности

$$
p_{T_{w}}(u, v)=\frac{a(v, w)}{\mathrm{m}_{T_{w}}(u)}= \begin{cases}p(u, v), & \text { если } u \in T_{w} \backslash\{w\}, v \sim u, \\ \frac{p(w, v)}{1-p\left(w, w^{-}\right)}, & \text {если } u=w, v \sim u .\end{cases}
$$

Имеем $F_{T_{w}}\left(u, u^{-}\right)=F\left(u, u^{-}\right)$и, значит, $F_{T_{w}}(u, w)=F(u, w)$ для каждой $u \in$ $T_{w} \backslash\{w\}$, поскольку до своего первого попадания в $w$ случайное блуждание на $T_{w}$ подчиняется тем же самым переходным вероятностям, что и первоначальное случайное блуждание на $T$. Легко видеть [63; с. 241], что случайное блуждание на $T_{w}$ будет невозвратным тогда и только тогда, когда для первоначального случайного блуждания выполнено неравенство $F\left(w, w^{-}\right)<1$, что в свою очередь выполняется тогда и только тогда, когда $\nu_{o}\left(\partial T_{w}\right)>0$. (В этом и в двух предыдущих пунктах это условие всегда предполагается выполненным, но для доказательства теоремы 6.4 мы предполагаем лишь, что случайное блуждание на всем $T$ является невозвратным.) Обратно, если $F\left(w, w^{-}\right)=1$, то $F(u, w)=1$ для всех $u \in T_{w}$. 
Ниже нам будет нужна следующая формула для предельных распределений.

Лемма 6.6. Для $u \in T \backslash\{о\}$ выполняется равенство

$$
\nu_{u}\left(\partial T_{u}\right)=1-p\left(u, u^{-}\right)\left(G(u, u)-G\left(u^{-}, u\right)\right) .
$$

ДокАЗАТЕЛЬСтво. В силу (7.7) имеем

$$
G(u, u) p\left(u, u^{-}\right)=\frac{F\left(u, u^{-}\right)}{1-F\left(u, u^{-}\right) F\left(u^{-}, u\right)} .
$$

Таким образом, после короткого вычисления с использованием (6.11) мы получаем

$$
p\left(u, u^{-}\right)\left(G(u, u)-G\left(u^{-}, u\right)\right)=\left(1-F\left(u^{-}, u\right)\right) G(u, u) p\left(u, u^{-}\right)=1-\nu_{u}\left(\partial T_{u}\right) .
$$

Лемма доказана.

ДокАЗАТЕЛЬСтво теоремы 6.4. Сначала докажем формулу Дуба-Наим в случае, когда $\varphi=\mathbf{1}_{\partial T_{v}}$ и $\psi=\mathbf{1}_{\partial T_{w}}$ для двух собственных ветвей $T_{v}$ и $T_{w}$ дерева $T$. Они либо не пересекаются, либо одна из них содержит другую.

Случай 1: $T_{w} \subset T_{v}$. (Случай $T_{v} \subset T_{w}$ аналогичен в силу симметрии.) Это означает, что $w \in T_{v}$. Для $x, y \in \partial T$ имеем

$$
(\varphi(x)-\varphi(y))(\psi(x)-\psi(y))=1,
$$

если $x \in \partial T_{w}$ и $y \in \partial T \backslash \partial T_{v}$ или наоборот, и

$$
(\varphi(x)-\varphi(y))(\psi(x)-\psi(y))=0
$$

в противном случае. В силу леммы 6.5 можно выбрать $v$ в качестве базовой точки. Таким образом, правая часть тождества равна

$$
\int_{\partial T \backslash \partial T_{v}} \int_{\partial T_{w}} \Theta_{v}(x, y) d \nu_{v}(x) d \nu_{v}(y)=\frac{\mathrm{m}(v)}{G(v, v)} \nu_{v}\left(\partial T \backslash \partial T_{v}\right) \nu_{v}\left(\partial T_{w}\right),
$$

так как $x \wedge_{v} y=v$ и $F(v, v)=1$.

Теперь обратимся к левой части формулы Дуба-Наим. Преобразования Пуассона функций $\varphi$ и $\psi$ даются формулами

$$
h_{\varphi}(u)=\nu_{u}\left(\partial T_{v}\right) \quad \text { и } \quad h_{\psi}(u)=\nu_{u}\left(\partial T_{w}\right) .
$$

В силу (6.11) имеем

$$
\begin{aligned}
h_{\varphi}(u) & =F(u, v) \nu_{v}\left(\partial T_{v}\right), & & u \in\{v\} \cup\left(T \backslash T_{v}\right), \\
1-h_{\varphi}(u) & =F(u, v) \nu_{v}\left(\partial T \backslash \partial T_{v}\right), & & u \in T_{v} .
\end{aligned}
$$

Положим $F_{v}(u)=F(u, v)$ и будем, везде, где это удобно, писать

$$
h_{\varphi}(u)-h_{\varphi}\left(u^{-}\right)=\left(1-h_{\varphi}\left(u^{-}\right)\right)-\left(1-h_{\varphi}(u)\right)
$$


и аналогично для $h_{\psi}$. Тогда получим

$$
\begin{gathered}
\mathscr{E}_{T}\left(h_{\varphi}, h_{\psi}\right)=\sum_{\left[u, u^{-}\right] \in E(T) \backslash E\left(T_{v}\right)} a\left(u, u^{-}\right)\left(F(u, v)-F\left(u^{-}, v\right)\right) \nu_{v}\left(\partial T_{v}\right) \\
\quad \times\left(F(u, v)-F\left(u^{-}, v\right)\right) \nu_{v}\left(\partial T_{w}\right) \\
\quad \sum_{\substack{\left[u, u^{-}\right] \in E\left(T_{v}\right) \backslash E\left(T_{w}\right) \\
\times\left(F(u, v)-F\left(u^{-}, v\right)\right) \nu_{v}\left(\partial T_{w}\right)}} a\left(u, u^{-}\right)\left(F(u, v)-F\left(u^{-}, v\right)\right) \nu_{v}\left(\partial T \backslash \partial T_{v}\right) \\
+\sum_{\left[u, u^{-}\right] \in E\left(T_{w}\right)} a\left(u, u^{-}\right)\left(F(u, v)-F\left(u^{-}, v\right)\right) \nu_{v}\left(\partial T \backslash \partial T_{v}\right) \\
\quad \times\left(F(u, v)-F\left(u^{-}, v\right)\right) \nu_{v}\left(\partial T \backslash \partial T_{w}\right) \\
=\mathscr{E}_{T}\left(F_{v}, F_{w}\right) \nu_{v}\left(\partial T_{v}\right) \nu_{v}\left(\partial T_{w}\right)-\mathscr{E}_{T_{v}}\left(F_{v}, F_{w}\right) \nu_{v}\left(\partial T_{w}\right) \\
+\mathscr{E}_{T_{w}}\left(F_{v}, F_{w}\right) \nu_{v}\left(\partial T \backslash \partial T_{v}\right)
\end{gathered}
$$

где, конечно, $\mathscr{E}_{T_{v}}$ - форма Дирихле случайного блуждания на ветви $T_{v}$, как обсуждалось выше, и аналогично для $\mathscr{E}_{T_{w}}$. Теперь $F_{v}=G_{v} / G(v, v)$ в силу (6.7), откуда следует, что (6.18) дает

$$
\mathscr{E}_{T}\left(F_{v}, F_{w}\right)=\frac{\mathscr{E}_{T}\left(G_{v}, F_{w}\right)}{G(v, v)}=\frac{\mathrm{m}(v) F(v, w)}{G(v, v)} .
$$

Напомним, что для случайного блуждания на $T_{v}$ имеем $F_{T_{v}}(u, v)=F(u, v)$ для каждой $u \in T_{v}$. Кроме того,

$$
\mathrm{m}_{T_{v}}(v)=\mathrm{m}(v)-a\left(v, v^{-}\right)=\mathrm{m}(v)\left(1-p\left(v, v^{-}\right)\right) .
$$

Применим (6.19) к этому случайному блужданию и получим

$$
\mathscr{E}_{T_{v}}\left(F_{v}, F_{w}\right)=\frac{\mathrm{m}(v)\left(1-p\left(v, v^{-}\right)\right) F(v, w)}{G_{T_{v}}(v, v)} .
$$

Применяя (6.8), (6.9) и

$$
p_{T_{v}}(v, u)=\frac{p(v, u)}{1-p\left(v, v^{-}\right)}
$$

для $u \in T_{v}$, получим

$$
\begin{aligned}
\frac{1-p\left(v, v^{-}\right)}{G_{T_{v}}(v, v)} & =1-p\left(v, v^{-}\right)-\left(1-p\left(v, v^{-}\right)\right) U_{T_{v}}(v, v) \\
& =1-p\left(v, v^{-}\right)-\sum_{u: u^{-}=v} p(v, u) F(u, v) \\
& =1-p\left(v, v^{-}\right)-\left(U(v, v)-p\left(v, v^{-}\right) F\left(v^{-}, v\right)\right) \\
& =\frac{1}{G(v, v)}-p\left(v, v^{-}\right)\left(1-F\left(v^{-}, v\right)\right) \\
& =\frac{\nu_{v}\left(\partial T_{v}\right)}{G(v, v)}
\end{aligned}
$$


где на последнем шаге мы использовали лемму 6.6. Отсюда следует, что

$$
\mathscr{E}_{T_{v}}\left(F_{v}, F_{w}\right)=\frac{\mathrm{m}(v) F(v, w)}{G(v, v)} \nu_{v}\left(\partial T_{v}\right) .
$$

Таким же образом, поменяв ролями $T_{w}$ и $T_{v}$ и используя свойство обратимости (6.13), выводим

$$
\begin{aligned}
\mathscr{E}_{T_{w}}\left(F_{v}, F_{w}\right) & =\frac{\mathrm{m}(w) F(w, v)}{G(w, w)} \nu_{w}\left(\partial T_{w}\right) \\
& =\frac{\mathrm{m}(v) F(v, w)}{G(v, v)} \nu_{w}\left(\partial T_{w}\right)=\frac{\mathrm{m}(v)}{G(v, v)} \nu_{v}\left(\partial T_{w}\right) .
\end{aligned}
$$

В итоге получаем, что

$$
\begin{aligned}
\mathscr{E}_{T}\left(h_{\varphi}, h_{\psi}\right) & =\mathscr{E}_{T_{w}}\left(F_{v}, F_{w}\right) \nu_{v}\left(\partial T \backslash \partial T_{v}\right) \\
& =\frac{\mathrm{m}(v)}{G(v, v)} \nu_{v}\left(\partial T_{w}\right) \nu_{v}\left(\partial T \backslash \partial T_{v}\right),
\end{aligned}
$$

как и предлагалось.

Случай 2: $T_{w} \cap T_{v}=\varnothing$. Ввиду леммы 6.5 обе части формулы Дуба-Наим не зависят от корня $о$. Таким образом, мы можем объявить корнем одного из соседей вершины $v$, который не лежит на $\pi(v, w)$. Кроме того, пусть $\bar{v}-$ сосед $v$ на $\pi(w, v)$. Тогда с выбранным новым корнем дополнение “старой” ветви $T_{v}$ есть ветвь $T_{\bar{v}}$, которая содержит $T_{w}$ (последняя остается той же самой относительно нового корня).

Таким образом, мы можем применить результат случая $1 \mathrm{k} T_{\bar{v}}$ и $T_{w}$. Это означает, что нужно заменить функции $\varphi$ и $h_{\varphi}$ соответственно функциями $1-\varphi$ и $1-h_{\varphi}$, что означает, что мы просто изменяем знак в обеих частях тождества. Таким образом, случай 2 сводится к случаю 1 без дальнейших вычислений.

Из того, что уже сделано, и из линейности преобразования Пуассона, а также из билинейности форм в обеих частях формулы Дуба-Наим мы выводим, что эта формула верна для линейных комбинаций индикаторных функций множеств $\partial T_{v}$. Эти индикаторные функции всюду плотны в пространстве $C(\partial T)$ относительно тах-нормы. Таким образом, формула Дуба-Наим выполняется для всех непрерывных функций на $\partial T$. Продолжение на всё $\mathscr{D}(\partial T, \mathscr{P})$ получается стандартной аппроксимацией. Теорема 6.4 доказана.

\section{7. Двойственность случайных блужданий на деревьях и изотропных процессов на их границах}

Сравнивая изотропные процессы и процессы Кигами [36] на $\partial T$, естественно задать следующие два вопроса.

Boпрос I. Для данного невозвратного случайного блуждания на $T$, связанного с формой Дирихле $\mathscr{E} T$ из (6.14), будет ли граничный процесс на $\partial T$, индуцированный формой $\mathscr{E} \mathscr{H} \mathscr{D}$ из $(6.16)$, совпадать с одним из изотропных процессов (1.8) на $\partial T$ с переходными вероятностями (6.6), индуцированным мерой 
$\mu=\nu_{o}$ на $\partial T$, некоторым ультраметрическим элементом $\phi$ на $T$ и подходящей функцией распределения расстояний $\sigma$ на $[0, \infty)$ ?

Вопрос II. Обратно, для данных $\mu, \phi$ и $\sigma$ существует ли случайное блуждание на $T$ с предельным распределением $\nu_{o}=\mu$ такое, что изотропный процесс, индуцированный $\mu, \phi$ и $\sigma$, является граничным процессом с формой Дирихле $\mathscr{E} \mathscr{H} \mathscr{D} ?$

Прежде чем отвечать на эти вопросы, нужно уточнить предположения. Когда мы начинаем с $(\phi, \mu, \sigma)$, мы, как и раньше, будем всегда предполагать, что $\mu$ имеет носителем всю $\partial T$.

Таким образом, от случайного блуждания мы также хотим, чтобы $\operatorname{supp}\left(\nu_{o}\right)=$ $\partial T$. Это равносильно требованию, что $\nu_{o}\left(\partial T_{v}\right)>0$ для каждого $v \in T$. В силу (6.11) это, в свою очередь, равносильно условию

$$
F\left(v, v^{-}\right)<1 \quad \text { для каждого } v \in T \backslash\{o\} .
$$

На самом деле мы увидим, что нам нужно немного большее, а именно, что

$$
\lim _{v \rightarrow \infty} G(v, o)=0
$$

т. е. для каждого $\varepsilon>0$ имеется конечное множество $A \subset T$ такое, что $G(v, o)<\varepsilon$ для всех $v \in T \backslash A$. Это условие необходимо и достаточно для разрешимости задачи Дирихле: для любого $\varphi \in C(\partial X)$ его преобразование Пуассона $h_{\varphi}$ дает единственное непрерывное продолжение $\varphi$ на $\widehat{T}$, являющееся гармонической функцией на T. См., например, [63; следствие 9.44].

Мы ограничимся рассмотрением случайных блужданий со свойствами (7.1) и (7.2) на корневом дереве с исходящими степенями $\geqslant 2$.

7.1. Ответ на вопрос I. Начнем со случайного блуждания, удовлетворяющего сформулированным выше требованиям. Мы знаем из раздела 1, что каждый $(\mu, \phi, \sigma)$-процесс возникает как стандартный процесс в смысле определения 2.9 относительно внутренней метрики (ср. с теоремой 2.10): для заданных $\phi$ и $\sigma$ внутренняя метрика индуцирована ультраметрическим элементом

$$
\phi_{*}(u)=-\frac{1}{\log \sigma(\phi(u))} .
$$

Таким образом, мы можем исключить $\sigma$ из наших рассмотрений, ограничиваясь теми ультраметрическими элементами $\phi$, для которых граничный процесс является стандартным процессом на $\partial T$, связанным с $\left(\phi, \nu_{0}\right)$.

Поскольку симметричные процессы определяются их формами Дирихле, мы выводим из теорем 3.12 и 6.4, что нам нужен такой ультраметрический элемент $\phi$, что $J(x, y)=\Theta_{o}(x, y)$ для всех $x, y \in \partial T$ при $x \neq y$, где $J(x, y)$ задано равенством (3.11). Переписывая $J(x, y)$ в терминах $\phi, \nu_{o}$ и структуры дерева, преобразуем это условие в следующее:

$$
\frac{1}{\phi(o)}+\int_{1 / \phi(o)}^{1 / \phi(x \wedge y)} \frac{d t}{\nu_{o}\left(B_{1 / t}^{\phi}(x)\right)}=\frac{\mathrm{m}(o)}{G(o, o) F(o, x \wedge y) F(x \wedge y, o)} .
$$


В нашем случае $\mathrm{m}(o)=1$, но из соображений общности мы пока не используем эту нормировку меры m. Прежде всего, так как $\operatorname{deg}^{+}(o) \geqslant 2$, существуют $x, y \in \partial T$ такие, что $x \wedge y=o$. Подставим эти две граничные точки в (7.4). Поскольку $F(o, o)=1$, видим, что должно выполняться равенство

$$
\Phi(o)=\frac{G(o, o)}{\mathrm{m}(o)} .
$$

Теперь возьмем $v \in T \backslash\{o\}$. Поскольку исходящие степени не меньше 2, существуют $x, y, y^{\prime} \in \partial T$ такие, что $x \wedge y=v$ и $x \wedge y^{\prime}=v^{-}$. Запишем (7.4) сначала для $\left(x, y^{\prime}\right)$, а затем для $(x, y)$, и возьмем разность, что приводит к уравнению

$$
\int_{1 / \phi\left(v^{-}\right)}^{1 / \phi(v)} \frac{d t}{\nu_{o}\left(B_{1 / t}^{\phi}(x)\right)}=\frac{\mathrm{m}(o)}{G(o, o) F(o, v) F(v, o)}-\frac{\mathrm{m}(o)}{G(o, o) F\left(o, v^{-}\right) F\left(v^{-}, o\right)} .
$$

В силу (6.5) в области интегрирования последнего интеграла должно выполняться равенство $B_{1 / t}^{\phi}(v)=\partial T_{v}$, откуда следует, что интеграл сводится к

$$
\left(\frac{1}{\phi(v)}-\frac{1}{\phi\left(v^{-}\right)}\right) \frac{1}{\nu_{o}\left(\partial T_{v}\right)}
$$

Умножим уравнение $(7.5)$ на $\nu_{o}\left(\partial T_{v}\right)$ и упростим полученную правую часть

$$
\left(\frac{\mathrm{m}(o)}{G(o, o) F(o, v) F(v, o)}-\frac{\mathrm{m}(o)}{G(o, o) F\left(o, v^{-}\right) F\left(v^{-}, o\right)}\right) \nu_{o}\left(\partial T_{v}\right),
$$

используя тождества (6.7)-(6.10) и первую из двух формул из (6.11) (для $\nu_{o}$ ). Получаем, что искомый ультраметрический элемент должен удовлетворять уравнению

$$
\frac{1}{\phi(v)}-\frac{1}{\phi\left(v^{-}\right)}=\frac{\mathrm{m}(o)}{G(v, o)}-\frac{\mathrm{m}(o)}{G\left(v^{-}, o\right)} \quad \text { для каждого } v \in T \backslash\{o\} .
$$

Это рекуррентно определяет $1 / \phi(v)$, и с условием $\mathrm{m}(o)=1$ получаем

$$
\phi(v)=G(v, o) .
$$

Поскольку в силу $(6.7)$ и (6.10) имеем

$$
G(v, o)=F\left(v, v^{-}\right) G\left(v^{-} o\right),
$$

предположения (7.1) и (7.2) дают, что $\phi$ - ультраметрический элемент. Обращая последние вычисления, мы видим, что при этом выборе $\phi$ действительно выполняется равенство $J(x, y)=\Theta_{o}(x, y)$ для всех $x, y \in \partial T$ при $x \neq y$. Таким образом, мы доказали следующее утверждение.

Теорема 7.1. Пусть $T$ - локально конечное корневое дерево с исходящими степенями $\geqslant 2$. Рассмотрим простое невозвратное случайное блуждание на T, для которого выполнены тождества (6.7) и (6.10). Тогда граничныи процесс на ӘТ, индуцированный формой Дирихле (6.16), совпадает со стандартным процессом, связанным с ультраметрическим элементом $\phi=G(\cdot, o)$ и предельным распределением $\nu_{о}$ случайного блуждания. 
Пусть $\mathscr{L}$ - лапласиан, связанный с граничным процессом из теоремы 7.1. Лапласиан $\mathscr{L}$ действует на локально постоянных функциях $f$ по правилу

$$
\left.\mathscr{L} f(x)=\int_{\partial T} f(x)-f(y)\right) \Theta_{o}(x, y) d \nu_{o}(y) .
$$

Ввиду отождествления шаров в $\partial T$ с вершинами дерева $T$, собственные функции из (3.6) теперь становятся такими:

$$
f_{v}=\frac{\mathbf{1}_{\partial T_{v}}}{\nu_{o}\left(\partial T_{v}\right)}-\frac{\mathbf{1}_{\partial T_{v^{-}}}}{\nu_{o}\left(\partial T_{v^{-}}\right)}, \quad v \in T \backslash\{o\} .
$$

Кроме того, положим $f_{o}=\mathbf{1}$ и заметим, что это собственная функция оператора $\mathscr{L}$ с собственным значением 0. Применяя теорему 3.8 , получаем следующее утверждение.

СлеДСТВИЕ 7.2. Для $v \in T \backslash\{o\}$ выполнено равенство $\mathscr{L} f_{v}=G\left(v^{-}, o\right)^{-1} f_{v}$ и множество собственных функииц $\left\{f_{v}\right\}_{v \in T}$ полно. В частности,

$$
\operatorname{spec} \mathscr{L}=\overline{\left\{G(v, o)^{-1}: v \in T\right\}} \cup\{0\} .
$$

ЗАмЕчАНИЕ 7.3. Для любых двух вершин $v$ и $w$ из $T \backslash\{o\}$ таких, что $v^{-}=$ $w^{-}=u$, функции $f_{v}$ и $f_{w}$ являются собственными функциями оператора $\mathscr{L}$, соответствующими собственному значению $\lambda=1 / G(u, o)$. Отсюда следует, что собственное пространство $\mathscr{H}(u)$, соответствующее вершине $u$, натянуто на функции $\left\{f_{v}: v^{-}=u\right\}$. Поскольку ранг системы $\left\{f_{v}: v^{-}=u\right\}$ равен $\operatorname{deg}^{+}(u)-1$, где $\operatorname{deg}^{+}(u) \geqslant 2$ - исходящая степень вершины $u$, получаем

$$
\operatorname{dim} \mathscr{H}(u)=\operatorname{deg}^{+}(u)-1
$$

(cp. c $(3.9))$.

ЗАмечАниЕ 7.4. Для заданного случайного блуждания на $T$ связанный с ним граничный процесс на $\partial T$ можно попробовать реализовать как $\left(\nu_{o}, \phi, \sigma\right)$ процесс для ультраметрического элемента $\phi$, отличного от $G(\cdot, o)$. Это означает, что нужно найти подходящее распределение расстояний $\sigma$ на $[0, \infty)$, отличное от обратного экспоненциального распределения (2.16). Ввиду 7.3 мы ищем такое $\sigma$, чтобы для нашего заданного $\phi$ общего вида выполнялось равенство

$$
\sigma(\phi(v))=e^{-1 / G(v, o)} .
$$

Для этого необходимо, чтобы выполнялось равенство $\phi(u)=\phi(v)$ для всех $u, v$ таких, что $G(u, o)=G(v, o)$, так как нам нужно, чтобы функция $\phi$ была постоянной на эквипотенциальных множествах. В этом случае функция распределения $\sigma(r)$ определяется вышеприведенным равенством для $r$, лежащих в множестве значений $\Lambda_{\phi}$ ультраметрики $d_{\phi}$. Затем эта функция может быть продолжена на $[0,+\infty)$. 
7.2. Ответ на вопрос II. Ответить на вопрос II означает, что мы начинаем с $\phi$ и $\mu$ и ищем случайное блуждание с предельным распределением $\nu_{o}=\mu$, для которого стандартный $(\phi, \mu)$-процесс является граничным процессом, связанным со случайным блужданием. Мы знаем из теоремы 7.1, что в этом случае должно выполняться равенство $\phi(v)=G(v, o)$, откуда, в частности, следует, что $\phi(o)>1$. Значит, нельзя ожидать, что подойдет любой ультраметрический элемент $\phi$. Наиболее естественный выбор состоит в том, чтобы заменить $\phi$ элементом $C \cdot \phi$ для некоторой постоянной $C>0$. Для стандартных процессов, связанных соответственно с $\phi$ и $C \cdot \phi$, это просто приведет к линейной замене времени: если старый процесс был $\left\{X_{t}\right\}_{t>0}$, то новый будет $\left\{X_{t / C}\right\}_{t>0}$.

ТеОрема 7.5. Пусть $T$ - локально конечное корневое дерево с исходящими степенями $\geqslant 2$. Рассмотрим ультраметрический элемент ф на $T$ и вероятностную меру н с полным носителем на ӘТ. Тогда существуют единственная постоянная $C>0$ и единственное простое невозвратное случайное блуждание на T, удовлетворяющее (6.7) и (6.10), со следующими свойствами:

1) $\mu=\nu_{о}$ - предельное распределение случайного блуждания;

2) граничный прочесс совпадает со стандартным процессом на ӘТ, индуиированным ультраметрическим элементом $C \cdot \phi$ и заданной мерой $\mu$.

Для доказательства нам понадобятся еще три формулы (первые две взяты из [63; лемма 9.35], а третья сразу следует из (6.11) и (6.10)):

$$
\begin{aligned}
G(u, u) p(u, v) & =\frac{F(u, v)}{1-F(u, v) F(v, u)}, \quad \text { если } u \sim v, \\
G(u, u) & =1+\sum_{v: v \sim u} \frac{F(u, v) F(v, u)}{1-F(u, v) F(v, u)}, \\
F\left(v^{-}, v\right) & =\frac{\nu_{o}\left(\partial T_{v}\right) / F\left(o, v^{-}\right)}{1-F\left(v, v^{-}\right)+F\left(v, v^{-}\right) \nu_{o}\left(\partial T_{v}\right) / F\left(o, v^{-}\right)} .
\end{aligned}
$$

ДоКАЗАТЕЛЬСтво тЕОРЕмы 7.5. Мы будем действовать следующим образом: начнем с $\phi$ и $\mu$ и заменим $\phi$ новым ультраметрическим элементом $C \cdot \phi$, где $C$ будет определено позже, а $\mu$ будет кандидатом на предельное распределение случайного блуждания, которое мы ищем.

Используя имеющиеся формулы, сначала построим единственным возможным образом величины $F(u, v), u, v \in T$, в частности, когда $u \sim v$. В свою очередь, они приводят к функции Грина $G(u, v)$. Пока это будут только "потенциальные" величины, годность которых нужно будет проверить. До этой проверки мы обозначаем их через $\widetilde{F}(u, v)$ и $\widetilde{G}(u, v)$. С помощью (7.7) они приведут к определению переходных вероятностей $p(u, v)$. Стохастичность получающихся переходных матриц $\mathscr{P}$ тоже нужно будет проверить.

Только тогда мы будем использовать рассуждения из теории потенциала, чтобы показать, что $\widetilde{G}(u, v)$ действительно является функцией Грина, связанной с $\mathscr{P}$, так что можно будет убрать знак вопроса, подразумевающийся в символе " ". 
Прежде всего, ввиду теоремы 7.1 должно выполняться равенство

$$
C \cdot \phi(v)=\widetilde{G}(v, o),
$$

откуда в силу (6.7) и (6.10) получаем

$$
\widetilde{F}\left(v, v^{-}\right)=\frac{\phi(v)}{\phi\left(v^{-}\right)} \quad \text { для } v \in T \backslash\{o\}
$$

и, более общо,

$$
\widetilde{F}(v, u)=\frac{\phi(v)}{\phi(u)}, \quad \text { когда } u \leqslant v .
$$

Сразу заметим, что $0<\widetilde{F}(v, u)<1$, когда $u<v$, и $\widetilde{F}(u, u)=1$.

Далее воспользуемся (7.9), чтобы рекуррентно построить $\widetilde{F}\left(v^{-}, v\right)$ и $\widetilde{F}(o, v)$. Начинаем с $\widetilde{F}(o, o)=1$. Если $v \neq o$ и $\widetilde{F}\left(o, v^{-}\right)$уже дано, причем

$$
\mu\left(\partial T_{v^{-}}\right) \leqslant \widetilde{F}\left(o, v^{-}\right) \leqslant 1
$$

(нижняя оценка требуется в силу (6.11)), то нужно положить

$$
\widetilde{F}\left(v^{-}, v\right)=\frac{\mu\left(\partial T_{v}\right) / \widetilde{F}\left(o, v^{-}\right)}{1-\widetilde{F}\left(v, v^{-}\right)+\widetilde{F}\left(v, v^{-}\right) \mu\left(\partial T_{v}\right) / \widetilde{F}\left(o, v^{-}\right)}
$$

и

$$
\widetilde{F}(o, v)=\widetilde{F}\left(o, v^{-}\right) \widetilde{F}\left(v^{-}, v\right) .
$$

Поскольку

$$
\widetilde{F}\left(o, v^{-}\right) \geqslant \mu\left(\partial T_{v^{-}}\right) \geqslant \mu\left(\partial T_{v}\right)
$$

видим, что

$$
0<\widetilde{F}\left(v^{-}, v\right) \leqslant 1
$$

Положим, как следует из (6.10),

$$
\widetilde{F}(o, v)=\widetilde{F}\left(o, v^{-}\right) \widetilde{F}\left(v^{-}, v\right) .
$$

Формула (7.11) преобразуется в следующую:

$$
\mu\left(\partial T_{v}\right)=\widetilde{F}\left(o, v^{-}\right) \widetilde{F}\left(v^{-}, v\right) \frac{1-\widetilde{F}\left(v, v^{-}\right)}{1-\widetilde{F}\left(v, v^{-}\right) \widetilde{F}\left(v^{-}, v\right)} \leqslant \widetilde{F}(o, v) \leqslant 1,
$$

как и требуется для нашего рекуррентного построения. На этот момент имеем все значения $\widetilde{F}(u, v)$, сначала при $u \sim v$, а потом и для всех $u, v$ с помощью взятия произведений вдоль геодезических путей.

Теперь можно вычислить постоянную $C$ : формула (7.8) в сочетании с $(7.10)$ и (7.12) при $u \sim o$ дает равенства

$$
\begin{aligned}
C \Phi(o) & =\widetilde{G}(o, o)=1+\sum_{u: u \sim o} \frac{\widetilde{F}(o, u) \widetilde{F}(u, o)}{1-\widetilde{F}(o, u) \widetilde{F}(u, o)} \\
& =1+\sum_{u: u \sim o} \frac{\widetilde{F}(u, o)}{1-\widetilde{F}(u, o)} \mu\left(\partial T_{u}\right) \\
& =1+\sum_{u: u \sim o} \frac{\phi(u) / \phi(o)}{1-\phi(u) / \phi(o)} \mu\left(\partial T_{u}\right) .
\end{aligned}
$$


Поэтому получаем

$$
C=\frac{1}{\phi(o)}+\sum_{u: u \sim o} \frac{\phi(u) / \phi(o)}{\phi(o)-\phi(u)} \mu\left(\partial T_{u}\right) .
$$

Теперь построим $\widetilde{G}(u, u)$ с помощью $(7.8)$ :

$$
\widetilde{G}(u, u)=1+\sum_{v: v \sim u} \frac{\widetilde{F}(u, v) \widetilde{F}(v, u)}{1-\widetilde{F}(u, v) \widetilde{F}(v, u)} .
$$

Для $u=o$ мы знаем, что это согласуется с нашим выбором $C$. Наконец, наш единственный выбор для функции Грина есть

$$
\widetilde{G}(u, v)=\widetilde{F}(u, v) \widetilde{G}(v, v), \quad u, v \in T .
$$

Теперь мы наконец приходим к единственно возможному способу определения переходных вероятностей с помощью (7.7):

$$
p(u, v)=\frac{1}{\widetilde{G}(u, u)} \frac{\widetilde{F}(u, v)}{1-\widetilde{F}(u, v) \widetilde{F}(v, u)} .
$$

УтВеРЖДЕНИЕ 7.1. Матрица Я является стохастической.

ДоКАЗАТЕЛЬСТво УТВЕРЖДЕНИЯ 7.1. Сочетая (7.15) с (7.14), выводим, что нужно проверить, что для каждого $u \in T$ выполняется равенство

$$
\sum_{v: v \sim u} \frac{\widetilde{F}(u, v)(1-\widetilde{F}(v, u))}{1-\widetilde{F}(u, v) \widetilde{F}(v, u)}=1 .
$$

Если $u=o$, то в силу (7.12) последнее равенство есть не что иное, как

$$
\sum_{v: v \sim o} \mu\left(\partial T_{v}\right)=1
$$

Если $u \neq o$, то снова в силу (7.12) левая часть в (7.16) есть

$$
\begin{aligned}
\sum_{v: v^{-}=u} & \frac{\widetilde{F}(u, v)(1-\widetilde{F}(v, u))}{1-\widetilde{F}(u, v) \widetilde{F}(v, u)}+\frac{\widetilde{F}\left(u, u^{-}\right)\left(1-\widetilde{F}\left(u^{-}, u\right)\right)}{1-\widetilde{F}\left(u, u^{-}\right) \widetilde{F}\left(u^{-}, u\right)} \\
= & \sum_{v: v^{-}=u} \frac{\mu\left(\partial T_{v}\right)}{\widetilde{F}(o, u)}+1-\frac{1-\widetilde{F}\left(u^{-}, u\right)}{1-\widetilde{F}\left(u, u^{-}\right) \widetilde{F}\left(u^{-}, u\right)}=1 .
\end{aligned}
$$

Это доказывает утверждение 7.1 .

УтВеРЖДЕНИЕ 7.2. Для любого $u_{0} \in T$ функиия $\widetilde{g}_{u_{0}}(u)=\widetilde{G}\left(u, u_{0}\right)$ удовлетворяет равенству $\mathscr{P} \widetilde{g}_{u_{0}}=\widetilde{g}_{u_{0}}-\mathbf{1}_{u_{0}}$.

ДокАЗАТЕЛЬСтво УТВЕРЖДЕНИЯ 7.2. Во-первых, сочетая (7.14) с (7.15), получаем

$$
\begin{aligned}
P \widetilde{g}_{u_{0}}\left(u_{0}\right) & =\sum_{v: v \sim u_{0}} p\left(u_{0}, v\right) \widetilde{F}\left(v, u_{0}\right) \widetilde{G}\left(u_{0}, u_{0}\right) \\
& =\sum_{v: v \sim u_{0}} \frac{\widetilde{F}\left(u_{0}, v\right) \widetilde{F}\left(v, u_{0}\right)}{1-\widetilde{F}\left(u_{0}, v\right) \widetilde{F}\left(v, u_{0}\right)}=\widetilde{g}_{u_{0}}\left(u_{0}\right)-1,
\end{aligned}
$$


и утверждение 7.2 верно при $u=u_{0}$. Во-вторых, при $u \neq u_{0}$ пусть $w-$ сосед вершины $u$ на $\pi\left(u, u_{0}\right)$. Тогда

$$
\begin{aligned}
P \widetilde{g}_{u_{0}}(u)= & \sum_{v: v \sim u, v \neq w} p(u, v) \widetilde{F}(v, u) \widetilde{G}\left(u, u_{0}\right)+p(u, w) \widetilde{G}\left(w, u_{0}\right) \\
= & \underbrace{\sum_{v \sim u} \frac{\widetilde{F}(u, v) \widetilde{F}(v, u)}{1-\widetilde{F}(u, v) \widetilde{F}(v, u)}}_{\widetilde{v}(u, u)-1} \frac{\widetilde{G}\left(u, u_{0}\right)}{\widetilde{G}(u, u)} \\
& \quad-p(u, w) \widetilde{F}(w, u) \widetilde{G}\left(u, u_{0}\right)+p(u, w) \widetilde{G}\left(w, u_{0}\right) \\
= & G\left(u, u_{0}\right)\left(1-\frac{1}{\widetilde{G}(u, u)}-p(u, w) \widetilde{F}(w, u)+p(u, w) \frac{1}{\widetilde{F}(u, w)}\right) \\
= & \widetilde{g}_{u_{0}}(u),
\end{aligned}
$$

так как

$$
\frac{p(u, w)}{\widetilde{F}(u, w)}-p(u, w) \widetilde{F}(w, u)=\frac{1}{\widetilde{G}(u, u)}
$$

в силу (7.15). Это завершает доказательство утверждения 7.2.

Теперь мы можем заключить, что функция $\widetilde{g}_{u_{0}}$ непостоянная, положительная и супергармоническая. Поэтому случайное блуждание с переходной матрицей $\mathscr{P}$, заданной формулой (7.15), является невозвратным и обладает функцией Грина $G(u, v)$. Кроме того, в силу теоремы Рисса о разложении имеем

$$
\widetilde{g}_{u_{0}}=G f+h
$$

где $h$ - неотрицательная гармоническая функция, а заряд $f$ потенциала

$$
G f(u)=\sum_{v} G(u, v) f(v)
$$

равен $f=\widetilde{g}_{u_{0}}-P \widetilde{g}_{u_{0}}=\mathbf{1}_{u_{0}}$, т. е.

$$
\widetilde{G}\left(u, u_{0}\right)=G\left(u, u_{0}\right)+h(x) \quad \text { для всех } u \in T \text {. }
$$

Пусть теперь $x \in \partial T$ и $v=u_{0} \wedge x$. Если $u \in T_{v}$, то в силу нашего построения имеем

$$
\widetilde{G}\left(u, u_{0}\right)=\widetilde{G}(u, o) \frac{\widetilde{G}\left(v, u_{0}\right)}{\phi(v)} \phi(u) \rightarrow 0 \quad \text { при } u \rightarrow x .
$$

Поэтому $\widetilde{G}\left(\cdot, u_{0}\right)$ обращается в нуль на бесконечности, и то же верно для $h$. В силу принципа максимума имеем $h \equiv 0$.

Мы заключаем, что $\widetilde{G}(u, v)=G(u, v)$ для всех $u, v \in T$. Но тогда в силу нашего построения также выполнено равенство $\widetilde{F}(u, v)=F(u, v)$.

Сравнивая (7.12) с (6.11), видим, что $\mu=\nu_{o}$. Это завершает доказательство теоремы 7.5 . 
7.3. Некомпактный случай. Возможности подхода настоящей работы не ограничиваются компактными пространствами. В случае некомпактного локально компактного ультраметрического пространства без изолированных точек дерево строится таким же образом: множество вершин соответствует множеству всех замкнутых шаров, а окрестность в полученном дереве определяется, как выше: если вершина $v$ соответствует шару $B$, то предшественник $v^{-}$- это вершина, соответствующая шару $B^{\prime}$ (см. определение 3.6$)$, и имеется ребро $\left[v^{-}, v\right]$. Теперь у каждой вершины есть предшественник (в то время как в компактном случае у корневой вершины его нет), а дерево имеет корень на бесконечности, т. е. ультраметрическим пространством становится $\partial^{*} T=\partial T \backslash\{\varpi\}$, где $\varpi-$ выбранный базовый конец дерева $T$ (см. рис. 5).

Теперь начнем с такой ситуации: заданы дерево $T$ и базовый конец $\varpi \in \partial T$, а предшественник $v^{-}=v_{\varpi}^{-}$вершины $v$ относительно $\varpi$ есть сосед вершины $v$ на геодезической $\pi(v, \varpi)$. Для данных двух элементов $w, z \in \widehat{T} \backslash\{\varpi\}$ их конфлюэнт $w \curlywedge z$ относительно $\varpi$ снова определяется как последний общий элемент на геодезических $\pi(\varpi, w)$ и $\pi(\varpi, z)$ (являющийся вершиной, если только не выполняется $v=w \in \partial^{*} T$; см. рис. 5). Снова естественно предполагать, что каждая вершина имеет по крайней мере два исходящих соседа.

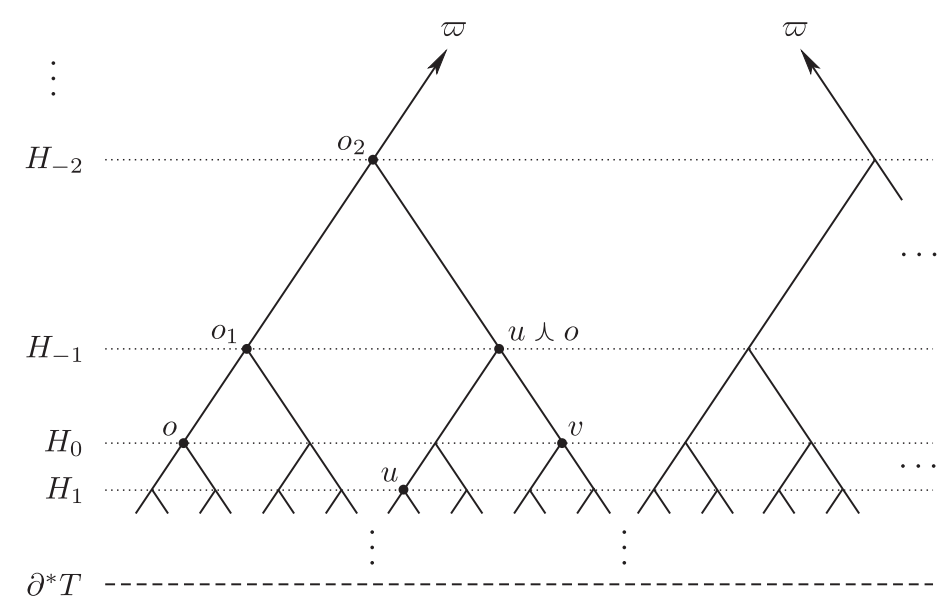

Рис. 5

В этой ситуации для определения 6.1 ультраметрического элемента $\phi: T \rightarrow$ $(0, \infty)$ нам нужно, кроме монотонности $\left[\phi(v)<\phi\left(v^{-}\right)\right]$, чтобы $\phi$ сходился к $\infty$ вдоль $\pi(o, \varpi)$, в то время как он должен стремиться к 0 вдоль любой геодезической, идущей на $\partial^{*} T$. Соответствующая ультраметрика на $\partial^{*} T$ тогда задается так же, как раньше:

$$
d_{\phi}(x, y)= \begin{cases}0, & \text { если } x=y, \\ \phi(x \curlywedge y), & \text { если } x \neq y .\end{cases}
$$

Заметим здесь, что когда $\phi$ не стремится к $\infty$ вдоль $\pi(x, \varpi)$, это также определяет ультраметрику, но тогда $\left(\partial^{*} T, d_{\phi}\right)$ не будет полным. Кроме того, если неравенство $\phi(v) \leqslant \phi\left(v^{-}\right)$не строгое, то получается ультраметрика, но тогда 
вышеприведенное построение дерева из замкнутых шаров не восстанавливает первоначальное дерево из $\left(\partial^{*} T, d_{\phi}\right)$. Наконец, если $\phi$ не стремится к 0 вдоль некоторой геодезической $\pi(o, x), x \in \partial^{*} T$, то $x$ будет изолированной точкой в $\left(\partial^{*} T, d_{\phi}\right)$. (Последние два наблюдения верны также в компактном случае для дерева с корневой вершиной.)

Возвращаясь к нашей постановке задачи, заметим, что эталонная мера $\mu$ $(\phi, \mu, \sigma)$-процесса может иметь бесконечную массу; это есть мера Радона с носителем, равным всему $\partial^{*} T$. Опять же мы знаем, что достаточно изучать стандартный $(\phi, \mu)$-процесс. Мы дадим краткое описание двойственности таких процессов и случайных блужданий на $T$. Это следует сравнить с последней частью второй работы Дж. Кигами [37] (препринт которой стал доступен, когда бо́льшая часть настоящей работы была сделана и, в частности, предварительная версия [64], содержащая разделы 6 и 7 настоящей работы, была уже в обращении).

По отношению к $\varpi$, ветвь дерева $T$ с корнем в $u \in T$ определяется следующим образом:

$$
T_{u}=T_{\varpi, u}=\{v \in T: u \in \pi(v, \varpi)\} .
$$

Тогда $\partial T_{u}-$ компактное подмножество в $\partial^{*} T$, а именно шар с $d_{\phi}$-диаметром $\phi(u)$. Здесь будет удобно обозначить через $T_{o, u}$ ветвь относительно корневой вершины $o \in T$ согласно (6.2). Заметим, что $T_{\varpi, u}=T_{o, u}$ тогда и только тогда,

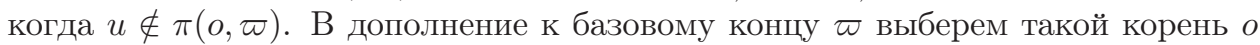
и обозначим через $o_{n}$ его $n$-го предшественника, т. е. вершину на $\pi(o, \varpi)$ на расстоянии $n$ от $о$.

Пусть теперь $\mathscr{P}=(p(u, v))_{u, v \in T}-$ переходная матрица простого невозвратного случайного блуждания на $T$. Снова предположим, что (7.1) выполняется: $F\left(v, v^{-}\right)<1$ для каждого $v \in T$, но теперь предшественники берутся относительно $\varpi$. (Действительно, отсюда следует (7.1) относительно любого выбора корневой вершины.) Теперь рассмотрим форму Дирихле $\mathscr{E} \mathscr{H} \mathscr{D}$ и формулу из теоремы 6.4. Нам хотелось бы переместить $о$ в точку $\varpi$ в этой формуле. Мы знаем из леммы 6.5 , что меры $\Theta_{o_{n}}(x, y) d \nu_{o_{n}}(x) d \nu_{o_{n}}(y)$ одинаковы для всех $n$. Однако меры $\nu_{o_{n}}$, будучи ограниченными на $\partial^{*} T$, обычно сходятся слабо к 0. Итак, проведем нормировку, полагая

$$
\mu_{n}=\frac{1}{\nu_{o_{n}}\left(\partial T_{o}\right)} \nu_{o_{n}} \quad \text { и } \quad J_{n}(x, y)=\Theta_{o_{n}}(x, y)\left(\nu_{o_{n}}\left(\partial T_{o}\right)\right)^{2} .
$$

Для следующей леммы напомним, что $T_{u}=T_{\varpi, u}$, и заметим, что $u \curlywedge o=o_{k}$ для некоторого $k \geqslant 0$.

Лемма 7.6. Пусть $A \subset \partial^{*} T$ компактно, так что имеется вершина и такая, что $A \subset \partial T_{u}$. Если и $о=o_{k}$, то для всех $n \geqslant k$ и всех $x, y \in \partial T_{o_{k}}$

$$
\mu_{n}(A)=\mu_{k}(A)=: \mu(A) \quad u \quad J_{n}(x, y)=J_{k}(x, y)=: J(x, y) .
$$

Имеем

$$
J(x, y)=\mathfrak{j}(x \curlywedge y) \quad n p u \mathfrak{j}(v)=\frac{\vartheta^{2}}{K(v, \varpi)^{2}} \frac{G(v, v)}{\mathrm{m}(v)}, \quad v \in T
$$


¿əe

$$
\vartheta=\frac{\mathrm{m}(o) \nu_{o}\left(\partial T_{o}\right)}{G(o, o)}
$$

$a$

$$
K(v, \varpi)=\frac{F(v, v \curlywedge o)}{F(o, v \curlywedge o)}=\frac{F\left(v, v \wedge_{o} \varpi\right)}{F\left(o, v \curlywedge_{o} \varpi\right)}
$$

- ядро Мартина в

ДоказАТЕЛЬство. Поскольку $\partial T_{o_{k}}$ содержит и $\partial T_{o}$, и $A$, при $n \geqslant k$ имеем

$$
\mu_{n}(A)=\frac{\nu_{o_{n}}(A)}{\nu_{o_{n}}\left(\partial T_{o}\right)}=\frac{F\left(o_{n}, o_{k}\right) \nu_{o_{k}}(A)}{F\left(o_{n}, o_{k}\right) \nu_{o_{k}}\left(\partial T_{o}\right)}=\mu_{k}(A) .
$$

Аналогично, пусть $x, y \in \partial T_{o_{k}}$ и пусть $x \curlywedge y=v$ - элемент из $T_{o_{k}}$. Используем тождество

$$
\mathrm{m}(v) G(v, w)=\mathrm{m}(w) G(w, v),
$$

из которого следует, что

$$
\mathrm{m}\left(o_{n}\right) F\left(o_{n}, o\right)=\mathrm{m}\left(o_{n}\right) \frac{G\left(o_{n}, o\right)}{G(o, o)}=\mathrm{m}\left(o_{n}\right) \frac{G\left(o, o_{n}\right)}{G(o, o)} .
$$

При $n \geqslant k$ имеем:

$$
\begin{aligned}
J_{n}(x, y) & =\frac{\nu_{o_{n}}\left(\partial T_{o}\right)^{2} \mathrm{~m}\left(o_{n}\right)}{F\left(o_{n}, v\right) G\left(v, o_{n}\right)}=\nu_{o}\left(\partial T_{o}\right)^{2} \frac{\mathrm{m}\left(o_{n}\right)^{2} F\left(o_{n}, o\right)^{2}}{\mathrm{~m}\left(o_{n}\right) F\left(o_{n}, v\right) G\left(v, o_{n}\right)} \\
& =\frac{\nu_{o}\left(\partial T_{o}\right)^{2} \mathrm{~m}(o)^{2}}{G(o, o)^{2}} \frac{G\left(o, o_{n}\right)^{2}}{G\left(v, o_{n}\right)^{2}} \frac{G(v, v)}{\mathrm{m}(v)},
\end{aligned}
$$

что дает предложенную формулу, поскольку

$$
G\left(o, o_{n}\right)=F(o, o \curlywedge v) G\left(o \curlywedge v, o_{n}\right), \quad G\left(v, o_{n}\right)=F(v, o \curlywedge v) G\left(o \curlywedge v, o_{n}\right) .
$$

Лемма доказана.

Теперь нетрудно доказать следующее утверждение.

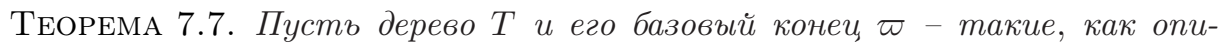
сано выше. Рассмотрим простое случайное блуждание на T, удовлетворяющее условию $F\left(v, v^{-}\right)<1$ для каждого $v \in T$. Пусть $\mu$ и $J$ - такие, как в лемме 7.6. Тогда для всех непрерывных функиий $\varphi, \psi$ на $\partial^{*} T$ с компактным носителем форму Дирихле (6.16) можно записать в виде

$$
\mathscr{E}_{\mathscr{H} \mathscr{D}}(\varphi, \psi)=\mathscr{E}_{J}(\varphi, \psi)+\vartheta \cdot \nu_{o}(\{\varpi\}) \int_{\partial^{*} T} \varphi(x) \psi(x) d \mu(x)
$$

¿əe

$$
\mathscr{E}_{J}(\varphi, \psi)=\frac{1}{2} \int_{\partial^{*} T} \int_{\partial^{*} T}(\varphi(x)-\varphi(y))(\psi(x)-\psi(y)) J(x, y) d \mu(x) d \mu(y)
$$


Если случайное блуждание регулярно, т.е. $\nu_{o}(\{\varpi\})=0$, то форма $\mathscr{E}_{J}=\mathscr{E}_{\mathscr{H}}$ индуиирует стандартный $(\mu, \phi)$-прощесс, где ультраметрический элемент $\phi$

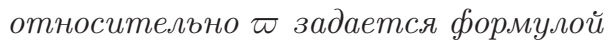

$$
\phi(v)=\frac{1}{\vartheta} K(v, \varpi)
$$

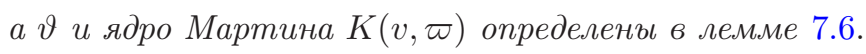

В частности, $(\mu, \phi)$-прочесс является граничным процессом с заменой времени.

ДокАзАтЕльство. Существует $k$ такое, что компактные носители функций $\varphi$ и $\psi$ содержатся в $\partial T_{o_{k}}$. Пусть $n \geqslant k$. Используя леммы 6.5 и 7.6, получаем

$$
\begin{aligned}
\mathscr{E}_{\mathscr{H} \mathscr{D}}(\varphi, \psi)= & \frac{1}{2} \int_{\partial T} \int_{\partial T}(\varphi(x)-\varphi(y))(\varphi(x)-\varphi(y)) J_{n}(x, y) d \mu_{n}(x) d \mu_{n}(y) \\
= & \frac{1}{2} \int_{\partial T_{o_{n}}} \int_{\partial T_{o_{n}}}(\varphi(x)-\varphi(y))(\varphi(x)-\varphi(y)) J(x, y) d \mu(x) d \mu(y) \\
& +\int_{\partial T_{o_{n}}} \varphi(x) \psi(x) \underbrace{\int_{\partial T \backslash \partial T_{o_{n}}} J_{n}(x, y) d \mu_{n}(y)}_{=: f_{n}(x)} d \mu(x) .
\end{aligned}
$$

При $n \rightarrow \infty$ имеем

$$
\frac{1}{2} \int_{\partial T_{o_{n}}} \int_{\partial T_{o_{n}}}(\varphi(x)-\varphi(y))(\varphi(x)-\varphi(y)) J(x, y) d \mu(x) d \mu(y) \rightarrow \mathscr{E}_{J}(\varphi, \psi)
$$

Рассмотрим второе слагаемое. Имеем

$$
f_{n}(x)=\int_{\partial T \backslash \partial T_{o_{n}}} \Theta_{o_{n}}(x, y) \nu_{o_{n}}\left(\partial T_{o}\right) d \nu_{o_{n}}(y) .
$$

Для $x \in \partial T_{o_{n}}$ и $y \in \partial T \backslash \partial T_{o_{n}}$ их конфлюэнт относительно точки $o_{n}$ есть сама точка $o_{n}$. Поэтому, используя (6.17) и (6.11), получаем

$$
\begin{aligned}
\Theta_{o_{n}}(x, y) \nu_{o_{n}}\left(\partial T_{o}\right) & =\frac{\mathrm{m}\left(o_{n}\right)}{G\left(o_{n}, o_{n}\right)} F\left(o_{n}, o\right) \nu_{o}\left(\partial T_{o}\right)=\frac{\mathrm{m}\left(o_{n}\right) G\left(o_{n}, o\right)}{G\left(o_{n}, o_{n}\right) G(o, o)} \nu_{o}\left(\partial T_{o}\right) \\
& =\frac{\mathrm{m}(o) G\left(o, o_{n}\right)}{G\left(o_{n}, o_{n}\right) G(o, o)} \nu_{o}\left(\partial T_{o}\right)=\frac{\mathrm{m}(o)}{G(o, o)} \nu_{o}\left(\partial T_{o}\right) F\left(o, o_{n}\right) \\
& =\vartheta F\left(o, o_{n}\right) .
\end{aligned}
$$


Теперь заметим, что для $y \in \partial T \backslash \partial T_{o_{n}}$ выполнено равенство $F\left(o, o_{n}\right) d \nu_{o_{n}}(y)=$ $d \nu_{o}(y)$. Поэтому

$$
f_{n}(x)=\vartheta \int_{\partial T \backslash \partial T_{o_{n}}} F\left(o, o_{n}\right) d \nu_{o_{n}}(y)=\vartheta \cdot \nu_{o}\left(\partial T \backslash \partial T_{o_{n}}\right) \rightarrow \vartheta \cdot \nu_{o}(\{\varpi\}),
$$

и при $n \rightarrow \infty$ можно воспользоваться теоремой о мажорируемой сходимости, чтобы получить, что

$$
\begin{aligned}
\int_{\partial T_{o_{n}}} \varphi(x) \psi(x) \int_{\partial T \backslash \partial T_{o_{n}}} J_{n}(x, y) d \mu_{n}(y) d \mu(x) & =\int_{\partial^{*} T} \varphi(x) \psi(x) f_{n}(x) d \mu(x) \\
& \rightarrow \vartheta \cdot \nu_{o}(\{\varpi\}) \int_{\partial^{*} T} \varphi(x) \psi(x) d \mu(x),
\end{aligned}
$$

как и предлагается. Чтобы доказать формулу для соответствующего ультраметрического элемента, действуем так же, как в доказательстве теоремы 7.1 (см. (7.4) и последующие строчки). Находим, что ультраметрический элемент должен удовлетворять уравнению

$$
\frac{1}{\Phi(v)}-\frac{1}{\Phi\left(v^{-}\right)}=\left(\mathfrak{j}(v)-\mathfrak{j}\left(v^{-}\right)\right) \mu\left(\partial T_{v}\right) .
$$

Правую часть этого уравнения можно вычислить: имеем $v^{-} \curlywedge o=o_{k}$ для некоторого $k \geqslant 0$ и, сочетая рассуждения после (7.4) с рассуждениями в доказательстве леммы 7.6, получаем

$$
\begin{aligned}
\left(\mathfrak{j}(v)-\mathfrak{j}\left(v^{-}\right)\right) \mu\left(\partial T_{v}\right) & =\left(\frac{\mathrm{m}\left(o_{k}\right)}{F\left(o_{k}, v\right) G\left(v, o_{k}\right)}-\frac{\mathrm{m}\left(o_{k}\right)}{F\left(o_{k}, v^{-}\right) G\left(v^{-}, o_{k}\right)}\right) \nu_{o_{k}}\left(\partial T_{v}\right) \nu_{o_{k}}\left(\partial T_{o}\right) \\
& =\left(\frac{\mathrm{m}\left(o_{k}\right)}{G\left(v, o_{k}\right)}-\frac{\mathrm{m}\left(o_{k}\right)}{G\left(v^{-}, o_{k}\right)}\right) F\left(o_{k}, o\right) \nu_{o}\left(\partial T_{o}\right) \\
& =\left(\frac{G\left(o, o_{k}\right)}{G\left(v, o_{k}\right)}-\frac{G\left(o, o_{k}\right)}{G\left(v^{-}, o_{k}\right)}\right) \frac{\mathrm{m}(o) \nu_{o}\left(\partial T_{o}\right)}{G(o, o)} \\
& =\frac{\vartheta}{K(v, \varpi)}-\frac{\vartheta}{K\left(v^{-}, \varpi\right)} .
\end{aligned}
$$

Следовательно, величина $1 / \Phi(\cdot)-\vartheta / K(\cdot, \varpi)$ должна быть постоянной. Поскольку случайное блуждание регулярно по Дирихле, имеем $K(v, \varpi) \rightarrow \infty$ при $v \rightarrow \varpi$. С другой стороны, $\Phi\left(o_{n}\right)$ также должно стремиться к бесконечности. Таким образом, постоянная равна 0 , и $\phi$ имеет предложенный вид. Теорема доказана.

Лемма 7.6 и теорема 7.7 ведут к более ясному пониманию и более простым доказательствам в [37; $\S 10,11]$, в частности в [37; теорема 11.3]. Именно, наша предельная мера $\mu$ совпадает с мерой $\nu_{*}$ из [37]. Заметим, что имеются примеры, где $\mu\left(\partial^{*} T\right)=\infty$, а также примеры, где $\mu\left(\partial^{*} T\right)<\infty$, несмотря на то, что ультраметрическое пространство некомпактно. 
ЗАмЕчАниЕ 7.8. В разделах 6 и 7 настоящей работы всегда предполагалось, что ультраметрическое пространство не имеет изолированных точек, что для дерева означает, что $\mathrm{deg}^{+} \geqslant 2$. Предмет работы [7] - противоположная ситуация, когда все точки изолированы, т.е. пространство дискретно. В таком случае ультраметрическое пространство также является границей дерева, которая состоит не из концов, а из терминальных вершин, т. е. вершин с одним соседом.

С точки зрения настоящего раздела смешанная ситуация так же хорошо поддается изучению. Если начать с локально компактного ультраметрического пространства, имеющего и изолированные, и неизолированные точки, можно таким же образом построить дерево. Множество вершин - это множество всех замкнутых шаров. Изолированные точки станут конечными вериинами дерева, у которых нет соседей, кроме предшественника, как, например, вершины $x$ и $y$ на рис. 6. Все внутренние (не конечные) вершины будут иметь исходящую степень $\geqslant 2$.
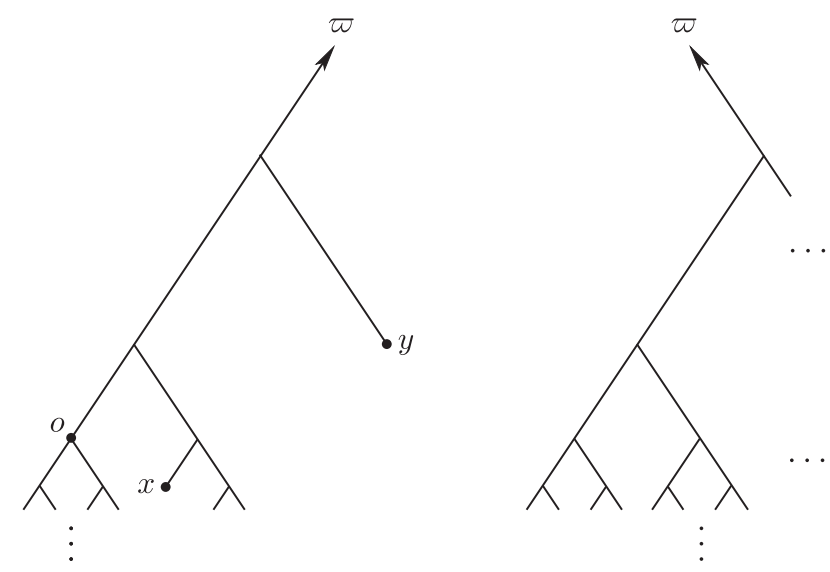

Рис. 6

В компактном случае граница $\partial T$ этого дерева состоит из терминальных вершин вместе с пространством концов. В некомпактном случае у нас опять будет

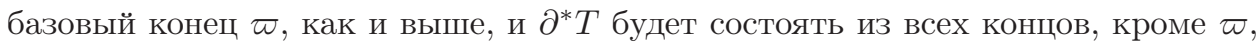
и из терминальных вершин. Определение ультраметрического элемента остается тем же, но нужно определять его только на внутренних вершинах. В этой общей постановке построение $(\phi, \mu, \sigma)$-процессов остается без изменений.

Даже при наличии изолированных точек двойственность между $(\phi, \mu, \sigma)$ процессами и случайными блужданиями на соответствующем дереве остается такой, как объяснялось выше. Случайное блуждание должно быть таким, что терминальные вершины поглощающие, а функция Грина стремится к 0 на бесконечности. Формула Дуба-Наим без труда продолжается на этот случай.

ЗАмечАниЕ 7.9. Рассмотрим снова общую ситуацию, когда мы начинаем с невозвратного случайного блуждания на локально конечном корневом дереве $T$. 
Предельное распределение $\nu_{o}$ в общем случае не будет иметь в качестве носителя всю границу $\partial T$. Граничный процесс, конечно, все еще можно построить (см. [36]), но он будет естественно существовать только на $\operatorname{supp}\left(\nu_{o}\right)$. Таким образом, можно считать, что наше ультраметрическое пространство - это только $\operatorname{supp}\left(\nu_{o}\right)$. В общем случае дерево, связанное с этим ультраметрическим пространством, не будет ни тем деревом, с которого мы начали, ни его невозвратным остовом, определяемым в [63; (9.27)] (как поддерево, индуцированное вершиной $о$ и всеми $v \in T \backslash\{o\}$ при $F\left(v, v^{-}\right)<1$, где $\left.v^{-}=v_{o}^{-}\right)$.

Тому имеется две причины. Во-первых, построение дерева по $\operatorname{supp}\left(\nu_{o}\right)$ никогда не вернет вершин с исходящей степенью 1. Во-вторых, какой-то конец, содержащийся в $\operatorname{supp}\left(\nu_{o}\right)$, может быть изолирован внутри этого множества, не будучи в то же время изолированным в $\partial T$. Но тогда этот элемент станет конечной вершиной в дереве, связанном с ультраметрическим (под)пространством $\operatorname{supp}\left(\nu_{o}\right)$. Это случается в точности тогда, когда невозвратный остов имеет изолированные концы.

Таким образом, следует работать с модифицированным "приведенным" деревом плюс случайное блуждание, чтобы сохранять двойственность между случайными блужданиями и изотропными процессами со скачками. Такие же наблюдения применимы к некомпактному случаю, с базовым концом вместо корня и мерой $\mu$ из леммы 7.6 вместо $\nu_{o}$.

ЗАмЕчАниЕ 7.10. Для заданного невозвратного случайного блуждания на корневом дереве $T$ в работе [36] восстанавливается также внутренняя метрика граничного процесса на $\partial T$ (компактный случай!) в терминах того, что называется ультраметрическим элементом в настоящей работе. Это, конечно, $\phi(x)=G(x, o)$, обозначаемый $D_{x}$ в [36], где показано, что для $\nu_{o}$-почти каждого $\xi \in \partial T$ имеет место сходимость $D_{x} \rightarrow 0$ вдоль геодезического луча $\pi(o, \xi)$. Это допускает следующую интерпретацию в терминах теории потенциала.

Точка $x \in \partial T$ называется регулярной для задачи Дирихле, если для каждой $\varphi \in C(\partial T)$ ее преобразование Пуассона $h_{\varphi}$ удовлетворяет соотношению

$$
\lim _{v \rightarrow x} h_{\varphi}(v)=\varphi(x)
$$

Из работы Д. И. Картрайта, П. М. Соарди и В. Вёсса [14; замечание 2] известно, что $x$ регулярна тогда и только тогда, когда $\lim _{u \rightarrow x} G(u, o)=0$ (как только $T$ имеет хотя бы 2 конца), см. также [63; теорема 9.43]. В силу той же теоремы из [63] множество регулярных точек имеет $\nu_{o}$-меру 1 , т. е. функция Грина обращается в нуль в $\nu_{o}$-почти каждой граничной точке.

ЗАмЕчАНиЕ 7.11. В доказательстве теоремы 7.5 мы восстанавливали переходные вероятности случайного блуждания исходя из $C \cdot \phi(u)=G(u, o)$ и $\mu=\nu_{o}$.

Похожий (но немного более простой) вопрос был рассмотрен З. Вондрачеком [60]: как восстановить переходные вероятности исходя из всех предельных распределений $\nu_{u}, u \in T$, на границе. Метод работы [60] и наш метод основаны на формулах (6.11) и (7.7)-(7.8), которые восходят к работе П. Картье [12]. 


\section{8. Случайное блуждание, связанное с $p$-адической дробной производной}

В этом разделе рассматривается пример, который объединяет подходы из раздела 5 и разделов 6,7 . Начнем с компактного случая.

8.1. Оператор $p$-адической дробной производной на $\mathbb{Z}_{p}$. Пусть $\mathbb{Z}_{p} \subset$ $\mathbb{Q}_{p}$ - группа $p$-адических целых чисел. В качестве аналога оператора $\mathfrak{D}^{\alpha}$ введем оператор $\mathbb{D}^{\alpha}$ дробной производной на $\mathbb{Z}_{p}$. Мы покажем, что это лапласиан подходящей изотропной марковской полугруппы. Затем мы построим случайное блуждание, связанное с $\mathbb{D}^{\alpha}$ в смысле разделов 6,7 .

Поскольку $\mathbb{Z}_{p}-$ компактная абелева группа, ее двойственная группа $\widehat{\mathbb{Z}}_{p}$ дискретная абелева группа. Известно, что группу $\widehat{\mathbb{Z}}_{p}$ можно отождествить с группой

$$
Z\left(p^{\infty}\right)=\left\{p^{-n} m: 0 \leqslant m<p^{n}, n=1,2, \ldots\right\},
$$

оснащенной сложением чисел по модулю 1 в качестве групповой операции. Для множеств (но не групп) имеет место включение $Z\left(p^{\infty}\right) \subset \mathbb{Q}_{p}$, откуда следует, что на группе $Z\left(p^{\infty}\right)$ корректно определена функция $\xi \mapsto\|\xi\|_{p}$.

ОпРЕДЕЛЕНИЕ 8.1. Оператор $\left(\mathbb{D}^{\alpha}, \mathscr{V}_{c}\right), \alpha>0$, определяется через преобразование Фурье на компактной абелевой группе $\mathbb{Z}_{p}$ равенством

$$
\widehat{\mathbb{D}^{\alpha} f}(\xi)=\|\xi\|_{p}^{\alpha} \widehat{f}(\xi), \quad \xi \in Z\left(p^{\infty}\right),
$$

где $\mathscr{V}_{c}$ - пространство локально постоянных функций на $\mathbb{Z}_{p}$.

Cp. с определением 5.1 оператора $\mathfrak{D}^{\alpha}$.

Непосредственно из этого определения следует, что оператор $\mathbb{D}^{\alpha}$ является неотрицательно определенным самосопряженным оператором, спектр которого совпадает с множеством значений функции

$$
\xi \mapsto\|\xi\|_{p}^{\alpha}: Z\left(p^{\infty}\right) \rightarrow \mathbb{R}_{+}
$$

т. e.

$$
\operatorname{spec} \mathbb{D}^{\alpha}=\left\{0, p^{\alpha}, p^{2 \alpha}, \ldots\right\} .
$$

Собственное пространство $\mathscr{H}(\lambda)$ оператора $\mathbb{D}^{\alpha}$, соответствующее собственному значению $\lambda=p^{k \alpha}, k \geqslant 1$, натянуто на функцию

$$
f_{k}=\frac{1}{\mu_{p}\left(p^{k} \mathbb{Z}_{p}\right)} \mathbf{1}_{p^{k} \mathbb{Z}_{p}}-\frac{1}{\mu_{p}\left(p^{k-1} \mathbb{Z}_{p}\right)} \mathbf{1}_{p^{k-1} \mathbb{Z}_{p}}
$$

и ее сдвиги $f_{k}(\cdot+a)$ с любыми $a \in \mathbb{Z}_{p} / p^{k} \mathbb{Z}_{p}$.

Действительно, вычисляя преобразование Фурье функции $f_{k}$ :

$$
\widehat{f_{k}}(\xi)=\mathbf{1}_{\left\{\|\xi\|_{p} \leqslant p^{k}\right\}}-\mathbf{1}_{\left\{\|\xi\|_{p} \leqslant p^{k-1}\right\}}=\mathbf{1}_{\left\{\|\xi\|_{p}=p^{k}\right\}},
$$

получаем

$$
\widehat{\mathbb{D}^{\alpha} f_{k}}(\xi)=\|\xi\|_{p}^{\alpha} \widehat{f}_{k}(\xi)=p^{k \alpha} \widehat{f}_{k}(\xi)
$$


Максимальное число линейно независимых функций в множестве $\left\{f_{k}(\cdot+a): a \in\right.$ $\left.\mathbb{Z}_{p} / p^{k} \mathbb{Z}_{p}\right\}$ есть $p^{k-1}(p-1)$, откуда вытекает равенство

$$
\operatorname{dim} \mathscr{H}(\lambda)=p^{k-1}(p-1) .
$$

Все вышесказанное показывает, что $\mathbb{D}^{\alpha}$ совпадает с лапласианом некоторой изотропной марковской полугруппы $\left(\mathbb{P}_{\alpha}^{t}\right)_{t>0}$ на ультраметрическом пространстве с мерой $\left(\mathbb{Z}_{p}, d_{p}, \mu_{p}\right)$. В частности, используя полное описание спектра spec $\mathbb{D}^{\alpha}$, вычислим внутреннее расстояние (мы обозначим его через $d_{p, \alpha}(x, y)$ ):

$$
d_{p, \alpha}(x, y)=\left(\frac{\|x-y\|_{p}}{p}\right)^{\alpha}
$$

Теперь легко вычислить спектральную функцию $\mathbb{N}_{\alpha}(x, \tau) \equiv \mathbb{N}_{\alpha}(\tau)$ и затем плотность скачков $\mathbb{J}_{\alpha}(x, y) \equiv \mathbb{J}_{\alpha}(x-y)$ оператора $\mathbb{D}^{\alpha}$. Покажем, что

$$
\mathbb{J}_{\alpha}(x, y)=\frac{p^{\alpha}-1}{1-p^{-\alpha-1}}\left(\frac{p^{-\alpha}-p^{-\alpha-1}}{1-p^{-\alpha}}+\frac{1}{\|x-y\|_{p}^{1+\alpha}}\right) .
$$

Напомним для сравнения, что в соответствии с (5.5) плотность скачков $J_{\alpha}(x, y)$ оператора $\mathfrak{D}^{\alpha}$ задается формулой

$$
J_{\alpha}(x, y)=\frac{p^{\alpha}-1}{1-p^{-\alpha-1}} \frac{1}{\|x-y\|_{p}^{1+\alpha}} .
$$

Чтобы доказать (8.1), вычислим $\mathbb{J}_{\alpha}(z)$. Пусть $\|z\|_{p}=p^{-l}$, тогда $d_{p, \alpha}(0, z)=$ $p^{-(l+1) \alpha}$ и

$$
\mathbb{J}_{\alpha}(z)=\int_{0}^{1 / d_{p, \alpha}(0, z)} \mathbb{N}_{\alpha}(\tau) d \tau=\int_{0}^{p^{(l+1) \alpha}} \mathbb{N}_{\alpha}(\tau) d \tau .
$$

Функция $\mathbb{N}_{\alpha}(\tau)$ - неубывающая непрерывная слева ступенчатая функция, имеющая скачки в точках $\tau_{k}=p^{k \alpha}, k=1,2, \ldots$, и принимающая в этих точках значения $\mathbb{N}_{\alpha}\left(\tau_{k}\right)=p^{k-1}$. Отсюда следует, что

$$
\begin{aligned}
\mathbb{J}_{\alpha}(z) & =1 \cdot p^{\alpha}+p\left(p^{2 \alpha}-p^{\alpha}\right)+p^{2}\left(p^{3 \alpha}-p^{2 \alpha}\right)+\cdots+p^{l}\left(p^{(l+1) \alpha}-p^{l \alpha}\right) \\
& =\frac{1-p^{-1}}{1-p^{-\alpha-1}}+\frac{p^{\alpha}-1}{1-p^{-\alpha-1}} p^{l(\alpha+1)} \\
& =\frac{p^{\alpha}-1}{1-p^{-\alpha-1}}\left(\frac{p^{-\alpha}-p^{-\alpha-1}}{1-p^{-\alpha}}+\frac{1}{\|z\|_{p}^{1+\alpha}}\right),
\end{aligned}
$$

как и требовалось. Далее применим теорему 3.12 и получим

$$
\mathbb{D}^{\alpha} f(x)=\int_{\mathbb{Z}_{p}}(f(x)-f(y)) \mathbb{J}_{\alpha}(x-y) d \mu_{p}(y) .
$$

Равенства (8.1), (8.2) и (5.1) теперь дают следующий результат. 
СлеДСтвиЕ 8.2. Для любой функиии $f$, определенной на $\mathbb{Z}_{p} \subset \mathbb{Q}_{p}$, положим $\widetilde{f}=f$ на $\mathbb{Z}_{p}$ и $\widetilde{f}=0$ в противном случае. Тогда

$$
f \in \operatorname{dom}\left(\mathbb{D}^{\alpha}\right) \Longrightarrow \tilde{f} \in \operatorname{dom}\left(\mathfrak{D}^{\alpha}\right)
$$

$u$

$$
\mathbb{D}^{\alpha} f(x)=\mathfrak{D}^{\alpha} \tilde{f}(x), \quad\left(\mathbb{D}^{\alpha} f, f\right)=\left(\mathfrak{D}^{\alpha} \tilde{f}, \tilde{f}\right),
$$

как только $x \in \mathbb{Z}_{p}, f \in \operatorname{dom}\left(\mathbb{D}^{\alpha}\right) u(\mathbf{1}, f)=0$.

8.2. Простое случайное блуждание на корневом дереве $\mathbb{T}_{p}^{o}$. Чтобы проиллюстрировать теорему 7.5, построим случайное блуждание на корневом дереве, связанном с $\mathbb{Z}_{p}$, граничный процесс которого совпадает с изотропным процессом, управляемым оператором $C \cdot \mathbb{D}^{\alpha}$, где $C=p^{-\alpha}\left(1-p^{-\alpha}\right)$.

Абелеву группу $\mathbb{Z}_{p}$ можно отождествить с границей дерева $\mathbb{T}_{p}^{o}$ с корнем $o$, где каждая вершина $v$ имеет $p$ исходящих соседей. В нашем отождествлении это дерево шаров ультраметрического пространства $\left(\mathbb{Z}_{p}, d_{p}\right)$ с корнем $о$, соответствующим всему $\mathbb{Z}_{p}$, и ультраметрикой $d_{p}(x, y)=\|x-y\|_{p}$. См. рис. 4 выше, где $p=2$. Зафиксируем постоянную $c \in(0,1)$ и рассмотрим простое случайное блуждание на $\mathbb{T}_{p}^{o}$ такое, что

$$
p\left(v, v^{-}\right)=1-c \quad \text { и } \quad p\left(v^{-}, v\right)= \begin{cases}1 / p, & \text { если } v^{-}=o \\ c / p & \text { в противном случае. }\end{cases}
$$

Используя [63; теорема 1.38 и предложение 9.3], можно точно вычислить функцию Грина $G(v, o)$, вероятности попадания $F(v, o)$ и другие величины, связанные с нашим случайным блужданием. В частности, выбирая $c=\left(1+p^{-\alpha}\right)^{-1}$, получим

$$
F(v, o)=p^{-\alpha|v|} \quad \text { и } \quad G(v, o)=\frac{p^{-\alpha|v|}}{1-p^{-\alpha}},
$$

где $|v|$ - расстояние на графе от $v$ до o. Видим, что функция Грина обращается в нуль на бесконечности, откуда следует, что случайное блуждание регулярно.

Переходные вероятности инвариантны относительно всех автоморфизмов дерева. Каждый такой автоморфизм должен оставлять на месте $о$ и каждый уровень дерева. Пусть $\nu=\nu_{o}$ - предельное распределение на $\partial \mathbb{T}_{p}^{o}$ случайного блуждания, начинающегося в $о$. Тогда $\nu$ также инвариантно под действием группы автоморфизмов дерева (действие которой продолжено на границу). $\mathrm{B}$ частности, оно инвариантно под действием $\mathbb{Z}_{p}$. Итак, при отождествлении $\partial \mathbb{T}_{p}^{o}$ с $\mathbb{Z}_{p}$ имеем, что $\nu=\mu_{p}$ есть нормированная мера Хаара группы $\mathbb{Z}_{p}$.

Теперь рассмотрим граничный процесс, индуцированный нашим случайным блужданием, как процесс со скачками на $\mathbb{Z}_{p}$. По теореме 7.1 граничный процесс возникает как изотропный процесс с эталонной мерой $\mu_{p}$. Пусть $\mathscr{L}-$ его лапласиан. В силу следствия 7.2 множество $\operatorname{spec} \mathscr{L}$ совпадает с множеством значений функции $v \mapsto 1 / G(v, o), v \in \mathbb{T}_{p}^{o}$, к которому добавлен $\{0\}$. Ввиду вышеприведенной формулы для $G(v, o)$ это означает, что

$$
\operatorname{spec} \mathscr{L}=\left\{0,\left(1-p^{-\alpha}\right), p^{\alpha}\left(1-p^{-\alpha}\right), p^{2 \alpha}\left(1-p^{-\alpha}\right), \ldots\right\} .
$$


Напомним, что

$$
\operatorname{spec} \mathbb{D}^{\alpha}=\left\{0, p^{\alpha}, p^{2 \alpha}, \ldots\right\}=\frac{p^{\alpha}}{1-p^{-\alpha}} \operatorname{spec} \mathscr{L} .
$$

Поскольку $\mathbb{D}^{\alpha}$ и $\mathscr{L}$ имеют одинаковые ортонормальные базисы из собственных функций, заключаем, что они пропорциональны, т. е.

$$
\mathbb{D}^{\alpha}=\frac{p^{\alpha}}{1-p^{-\alpha}} \mathscr{L}
$$

Таким образом, мы приходим к следующему заключению.

ПРеДЛОЖЕНИЕ 8.3. Граничный процесс $\left\{X_{t}\right\}_{t>0}$, связанный с определенньм в (8.4) случайнъм блужданием с параметром $c=\left(1+p^{-\alpha}\right)^{-1}$, и изотропный процесс $\left\{X_{t}^{\alpha}\right\}_{t>0}$, управляемый оператором $\mathbb{D}^{\alpha}$, связаны линейной заменой времени: $X_{t / C}=X_{t}^{\alpha}$, где $C=p^{-\alpha}\left(1-p^{-\alpha}\right)$.

Из равенства (8.6) следует, что плотность скачков $\mathbb{J}_{\alpha}(x, y)$ оператора $\mathbb{D}^{\alpha}$ и ядро Дуба-Наим $\Theta_{o}(x, y)$ оператора $\mathscr{L}$ связаны равенством

$$
\mathbb{J}_{\alpha}(x, y)=\frac{p^{\alpha}}{1-p^{-\alpha}} \Theta_{o}(x, y) .
$$

В заключение покажем, как вычислить ядро Дуба-Наим

$$
\Theta_{o}(x, y)=\frac{1}{G(o, o) F(o, v) F(v, o)}, \quad \text { где } v=x \wedge y,
$$

напрямую, используя данные из (8.5). У нас еще нет $F(o, v)$. Имеем

$$
N(v)=\frac{1}{F(o, v) F(v, o)} .
$$

Поскольку эта величина зависит только от уровня $k$ вершины $v$, рассмотрим произвольный геодезический луч $\left[o=v_{0}, v_{1}, \ldots\right]$ и построим линейную рекурсию для $N\left(v_{k}\right)$. Обозначая через $w_{1}$ произвольного соседа вершины $о$, отличного от $v_{1}$, и применяя [63; предложение $\left.9.3(\mathrm{~b})\right]$ и $(8.5)$, получим

$$
F\left(o, v_{1}\right)=\frac{1}{p}+\frac{p-1}{p} F\left(w_{1}, o\right) F\left(o, v_{1}\right)=\frac{1}{p}+\frac{(p-1) p^{-\alpha}}{p} F\left(o, v_{1}\right),
$$

откуда следует, что

$$
F\left(o, v_{1}\right)=\frac{p^{\alpha}}{p^{\alpha+1}-p+1} .
$$

Итак, получаем начальные значения

$$
N\left(v_{0}\right)=1 \quad \text { и } \quad N\left(v_{1}\right)=p^{\alpha+1}-p+1 .
$$

Далее, при $k \geqslant 1$ пусть $w_{k+1}-$ исходящий сосед вершины $v_{k}$, отличный от $v_{k+1}$. Снова применяя [63; предложение $9.3(\mathrm{~b})]$ и $(8.5)$, получим

$$
\begin{gathered}
F\left(v_{k}, v_{k+1}\right)=\frac{p^{\alpha}}{p\left(p^{\alpha}+1\right)}+\frac{(p-1) p^{\alpha}}{p\left(p^{\alpha}+1\right)} F\left(w_{k+1}, v_{k}\right) F\left(v_{k}, v_{k+1}\right) \\
+\frac{1}{p^{\alpha}+1} F\left(v_{k-1}, v_{k}\right) F\left(v_{k}, v_{k+1}\right)
\end{gathered}
$$


Подставим значение $F\left(w_{k+1}, v_{k}\right)=p^{-\alpha}$ и разделим на

$$
F\left(o, v_{k+1}\right)=F\left(o, v_{k}\right) F\left(v_{k}, v_{k+1}\right)=F\left(o, v_{k-1}\right) F\left(v_{k-1}, v_{k}\right) F\left(v_{k}, v_{k+1}\right) .
$$

Тогда

$$
\frac{1}{F\left(o, v_{k}\right)}=\frac{p^{\alpha}}{p\left(p^{\alpha}+1\right)} \frac{1}{F\left(o, v_{k+1}\right)}+\frac{p-1}{p\left(p^{\alpha}+1\right)} \frac{1}{F\left(o, v_{k}\right)}+\frac{1}{p^{\alpha}+1} \frac{1}{F\left(o, v_{k-1}\right)} .
$$

Умножая обе части последнего равенства на $1 / F\left(v_{k}, o\right)=p^{\alpha k}$, получим

$$
N\left(v_{k}\right)=\frac{1}{p\left(p^{\alpha}+1\right)} N\left(v_{k+1}\right)+\frac{p-1}{p\left(p^{\alpha}+1\right)} N\left(v_{k}\right)+\frac{p^{\alpha}}{p^{\alpha}+1} N\left(v_{k-1}\right) .
$$

Это однородная линейная рекурсия второго порядка с постоянными коэффициентами. Ее характеристический многочлен имеет корни 1 и $p^{\alpha+1}$. Поэтому

$$
N\left(v_{k}\right)=A+B p^{(\alpha+1) k} .
$$

Подставляя начальные значения, без труда находим значения $A$ и $B$. Чтобы получить ядро Дуба-Наим, нужно умножить на $1 / G(o, o)=1-p^{-\alpha}$. Итак,

$$
\Theta_{o}(x, y)=\frac{\left(1-p^{-\alpha}\right)(p-1)}{p^{\alpha+1}-1}+\frac{\left(1-p^{-\alpha}\right)\left(p^{\alpha+1}-p\right)}{p^{\alpha+1}-1} p^{(\alpha+1) k}=\frac{1-p^{-\alpha}}{p^{\alpha}} \mathbb{J}_{\alpha}(x, y),
$$

что и требовалось.

8.3. Случайное блуждание, соответствующее $\mathfrak{D}^{\alpha}$ на $\mathbb{Q}_{p}$. Мы построим случайное блуждание, соответствующее дробной производной на всем $\mathbb{Q}_{p}$, используя лемму 7.6 и теорему 7.7 о двойственности изотропных процессов и случайных блужданий в некомпактном случае.

Дерево, связанное с $\mathbb{Q}_{p}$, является однородным деревом $T=\mathbb{T}_{p}$ со степенями $p+1$. Нужно выбрать базовый конец $\varpi$. Тогда можно отождествить его

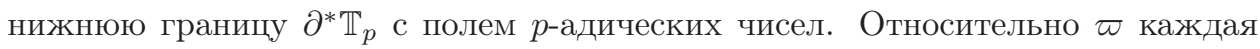
вершина $v$ имеет предшественника $v^{-}$и $p$ последователей. Каждое поддерево $T_{v}=T_{\varpi, v}$ изоморфно корневому дереву $\mathbb{T}_{p}^{o}$, рассмотренному выше в компактном случае $p$-адических целых чисел. В частности, выберем такую корневую вершину $о$, что $\partial T_{o}=\mathbb{Z}_{p}$. См. рис. 5 выше с $p=2$.

Теперь определим случайное блуждание на $\mathbb{T}_{p}$, как в (8.4), но с предшественниками относительно :

$$
p\left(v, v^{-}\right)=1-c \quad \text { и } \quad p\left(v^{-}, v\right)=\frac{c}{p}, \quad \text { где } \quad c=\left(1+p^{-\alpha}\right)^{-1} .
$$

Касательно следующих величин см., например, $\left[62 ;\right.$ с. 423, 424]. Для всех $v \in \mathbb{T}_{p}$

$$
F\left(v, v^{-}\right)=p^{-\alpha}, \quad F\left(v^{-}, v\right)=p^{-1}, \quad G(v, v)=\frac{1+p^{-\alpha}}{1-p^{-\alpha-1}}
$$

и

$$
\nu_{v}\left(\partial T_{v}\right)=\frac{1-p^{-\alpha}}{1-p^{-\alpha-1}}
$$




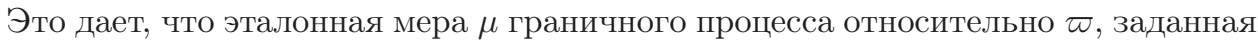
леммой 7.6, является стандартной мерой Хаара на $\mathbb{Q}_{p}$.

Вычислим $\vartheta=\left(1-p^{-\alpha}\right) /\left(1+p^{-\alpha}\right)$. Кроме того, положим $\mathfrak{h}(v)=d(v, v \curlywedge o)-$ $d(o, v \curlywedge o)$ (где $d$ - графовая метрика). Это орициклическое число вершины $v$. То есть вершины с $\mathfrak{h}(v)=k, k \in \mathbb{Z}$, являются элементами $k$-го поколения $H_{k}$ дерева (см. рис. 5), а $\partial T_{v}$ соответствует шару радиуса $p^{-k}$ в стандартной ультраметрике на $\mathbb{Q}_{p}$. Тогда

$$
K(v, \varpi)=p^{\alpha \mathfrak{h}(v)} \quad \text { и } \quad \mathrm{m}(v)=p^{(\alpha-1) \mathfrak{h}(v)} .
$$

Объединяя вышесказанное, получаем

$$
\Phi(v)=\frac{1+p^{-\alpha}}{1-p^{-\alpha}} p^{-\alpha \mathfrak{h}(v)} \quad \text { и } \quad \mathfrak{j}(v)=\frac{\left(1-p^{-\alpha}\right)^{2}}{\left(1+p^{-\alpha}\right)\left(1-p^{-\alpha-1}\right)} p^{(\alpha+1) \mathfrak{h}(v)} .
$$

Переводя это обратно в $p$-адические обозначения, заключаем, что внутренняя

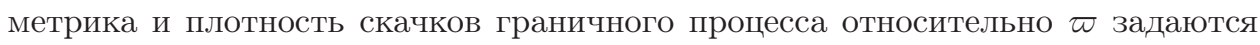
формулами

$$
\begin{aligned}
d_{\phi}(x, y) & =\frac{1-p^{-\alpha}}{1+p^{-\alpha}}\|x-y\|_{p}^{\alpha}, \\
J(x, y) & =\frac{\left(1-p^{-\alpha}\right)^{2}}{\left(1+p^{-\alpha}\right)\left(1-p^{-\alpha-1}\right)} \frac{1}{\|x-y\|_{p}^{\alpha+1}}=\frac{1-p^{-\alpha}}{p^{\alpha}+1} J_{\alpha}(x, y),
\end{aligned}
$$

где $J_{\alpha}-$ плотность скачков, связанная с $\mathfrak{D}^{\alpha}$. Итак, мы наконец получаем следующее утверждение.

ПреДЛОЖенИЕ 8.4. Граничный процесс $\left\{X_{t}\right\}_{t>0}$ относительно базового кониа ш, связанныи со случайным блужданием (8.8) на $\mathbb{T}_{p}$, и изотропный процесс со скачками $\left\{X_{t}^{\alpha}\right\}_{t>0}$, управляемый оператором $\mathfrak{D}^{\alpha}$ на $\mathbb{Q}_{p}$, связаны линейной заменой времени: $X_{t / C^{*}}=X_{t}^{\alpha}$, где $C^{*}=\left(1-p^{-\alpha}\right) /\left(p^{\alpha}+1\right)$.

\section{Список литературы}

[1] S. Albeverio, W. Karwowski, "Diffusion on p-adic numbers", Gaussian random fields (Nagoya, 1990), Ser. Probab. Statist., 1, World Sci. Publ., River Edge, NJ, 1991, 86-99.

[2] S. Albeverio, W. Karwowski, "A random walk on $p$-adics - the generator and its spectrum", Stochastic Process. Appl., 53:1 (1994), 1-22.

[3] S. Albeverio, X. Zhao, "On the relation between different constructions of random walks on p-adics", Markov Process. Related Fields, 6:2 (2000), 239-255.

[4] D. Aldous, S. N. Evans, "Dirichlet forms on totally disconnected spaces and bipartite Markov chains", J. Theoret. Probab., 12:3 (1999), 839-857.

[5] A. Bendikov, B. Bobikau, Ch. Pittet, "Some spectral and geometric aspects of countable groups", Random walks, boundaries and spectra, Progr. Probab., 64, Birkhäuser/Springer Basel AG, Basel, 2011, 227-234. 
[6] A. Bendikov, B. Bobikau, Ch. Pittet, "Spectral properties of a class of random walks on locally finite groups", Groups Geom. Dyn., 7:4 (2013), 791-820.

[7] A. Bendikov, A. Grigor'yan, Ch. Pittet, "On a class of Markov semigroups on discrete ultra-metric spaces", Potential Anal., 37:2 (2012), 125-169.

[8] A. Bendikov, P. Krupski, "On the spectrum of the hierarchical Laplacian", Potential Anal., 2014 (to appear); 2013, 27 pp., arXiv: 1308.4883v2.

[9] A. Bendikov, L. Saloff-Coste, Random walks on some countable groups, preprint, 2011.

[10] C. Berg, G. Forst, Potential theory on locally compact abelian groups, Ergeb. Math. Grenzgeb., 87, Springer-Verlag, New York-Heidelberg, 1975, vii+197 pp.

[11] S. Brofferio, W. Woess, "On transience of card shuffling", Proc. Amer. Math. Soc., 129:5 (2001), 1513-1519.

[12] P. Cartier, "Fonctions harmoniques sur un arbre", Convegno di Calcolo delle Probabilità (INDAM, Rome, 1971), Symposia Mathematica, 9, Academic Press, London, 1972, 203-270.

[13] D. I. Cartwright, "Random walks on direct sums of discrete groups", J. Theoret. Probab., 1:4 (1988), 341-356.

[14] D. I. Cartwright, P. M. Soardi, W. Woess, "Martin and end compactifications of nonlocally finite graphs", Trans. Amer. Math. Soc., 338:2 (1993), 679-693.

[15] Z.-Q. Chen, M. Fukushima, J. Ying, "Traces of symmetric Markov processes and their characterizations", Ann. Probab., 34:3 (2006), 1052-1102.

[16] T. Coulhon, A. Grigor'yan, Ch. Pittet, "A geometric approach to on-diagonal heat kernel low bounds on groups", Ann. Inst. Fourier (Grenoble), 51:6 (2001), 1763-1827.

[17] D. A. Darling, P. Erdős, "On the recurrence of a certain chain", Proc. Amer. Math. Soc., 19:2 (1968), 336-338.

[18] C. Dellacherie, S. Martínez, J. San Martín, "Ultrametric matrices and induced Markov chains", Adv. in Appl. Math., 17:2 (1996), 169-183.

[19] C. Dellacherie, S. Martínez, J. San Martín, "Ultrametric and tree potential", J. Theoret. Probab., 22:2 (2009), 311-347.

[20] J. L. Doob, "Boundary properties for functions with finite Dirichlet integrals", Ann. Inst. Fourier (Grenoble), 12 (1962), 573-621.

[21] J. Douglas, "Solution of the problem of Plateau", Trans. Amer. Math. Soc., 33:1 (1931), 263-321.

[22] S. N. Evans, "Local properties of Lévy processes on a totally disconnected group", J. Theoret. Probab., 2:2 (1989), 209-259.

[23] S. N. Evans, "Local field Brownian motion", J. Theoret. Probab., 6:4 (1993), 817-850.

[24] S. N. Evans, "Local fields, Gaussian measures, and Brownian motions", Topics in probability and Lie groups: boundary theory, CRM Proc. Lecture Notes, 28, Amer. Math. Soc., Providence, RI, 2001, 11-50.

[25] Н. Ферейг, С. А. Молчанов, "Случайные блуждания на абелевых группах с бесконечным числом образующих", Вестн. Моск. ун-та. Сер. 1. Матем. Мех., 1978, № 5, 22-29; англ. пер.: N. Fereig, S. A. Molchanov, "Random walks on abelian groups with an infinite number of generators", Moscow Univ. Math. Bull., 33:5 (1978), 17-23.

[26] L. Flatto, J. Pitt, "Recurrence criteria for random walks on countable Abelian groups", Illinois J. Math., 18:1 (1974), 1-19.

[27] M. Fukushima, Y. Oshima, M. Takeda, Dirichlet forms and symmetric Markov processes, 2nd ed., de Gruyter Stud. Math., 19, Walter de Gruyter \& Co., Berlin, 2011, $\mathrm{x}+489 \mathrm{pp}$. 
[28] M. Gromov, "Asymptotic invariants of infinite groups", Geometric group theory, v. 2 (Sussex, 1991), London Math. Soc. Lecture Note Ser., 182, Cambridge Univ. Press, Cambridge, 1993, 1-295.

[29] S. Haran, "Riesz potentials and explicit sums in arithmetic", Invent. Math., 101:1 (1990), 697-703.

[30] S. Haran, "Analytic potential theory over the p-adics", Ann. Inst. Fourier (Grenoble), 43:4 (1993), 905-944.

[31] E. Hewitt, K. A. Ross, Abstract harmonic analysis, v. 1: Structure of topological groups, integration theory, group representations, Grundlehren Math. Wiss., 115, Academic Press, New York; Springer-Verlag, Berlin-Göttingen-Heidelberg, 1963, viii+519 pp.

[32] B. Hughes, "Trees and ultrametric spaces: a categorical equivalence", Adv. Math., 189:1 (2004), 148-191.

[33] Р. С. Исмагилов, "О спектре самосопряженного оператора в $L_{2}(K)$, где $K$ - локальное поле; аналог формулы Фейнмана-Каца", ТМФ, 89:1 (1991), 18-24; англ. пер.: R. S. Ismagilov, "On the spectrum of a self-adjoint operator in $L_{2}(K)$, where $K$ is a local field; an analogue of the Feynman-Kac formula", Theoret. and Math. Phys., 89:1 (1991), 1024-1028.

[34] М. А. Касымджанова, "Рекуррентность инвариантных цепей Маркова на классе абелевых групп”, Вестн. Моск. ун-та. Сер. 1 Матем. Мех., 1981, № 3, 3-7; англ. пер.: M. A. Kasymdzhanova, "Recurrence of invariant Markov chains on a class of abelian groups", Moscow Univ. Math. Bull., 36:3 (1981), 1-6.

[35] H. Kesten, F. Spitzer, "Random walks on countably infinite Abelian groups", Acta Math., 114:1 (1965), 237-265.

[36] J. Kigami, "Dirichlet forms and associated heat kernels on the Cantor set induced by random walks on trees", Adv. Math., 225:5 (2010), 2674-2730.

[37] J. Kigami, "Transitions on a noncompact Cantor set and random walks on its defining tree", Ann. Inst. Henri Poincaré Probab. Statist., 49:4 (2013), 1090-1129.

[38] А.Н. Кочубей, "Параболические уравнения над полем $p$-адических чисел", Изв. АН СССР. Сер. матем., 55:6 (1991), 1312-1330; англ. пер.: А. N. Kochubeй, "Parabolic equations over the field of p-adic numbers", Math. USSR-Izv., 39:3 (1992), 1263-1280.

[39] A. N. Kochubei, Pseudo-differential equations and stochastics over non-Archimedean fields, Monogr. Textbooks Pure Appl. Math., 244, Marcel Dekker Inc., New York, 2001, xii+316 pp.

[40] G. F. Lawler, "Recurrence and transience for a card shuffling model", Combin. Probab. Comput., 4:2 (1995), 133-142.

[41] S. Martínez, D. Remenik, J. San Martín, "Level-wise approximation of a Markov process associated to the boundary of an infinite tree", J. Theoret. Probab., 20:3 (2007), 561-579.

[42] M. Del Muto, A. Figà-Talamanca, "Diffusion on locally compact ultrametric spaces", Expo. Math., 22:3 (2004), 197-211.

[43] M. Del Muto, A. Figà-Talamanca, "Anisotropic diffusion on totally disconnected abelian groups", Pacific J. Math., 225:2 (2006), 221-229.

[44] L. Naïm, "Sur le rôle de la frontière de R.S. Martin dans la théorie du potentiel", Ann. Inst. Fourier (Grenoble), 7 (1957), 183-281.

[45] J. Pearson, J. Bellissard, "Noncommutative Riemannian geometry and diffusion on ultrametric Cantor sets", J. Noncommut. Geom., 3:3 (2009), 447-480. 
[46] Ch. Pittet, L. Saloff-Coste, "Amenable groups, isoperimetric profiles and random walks", Geometric group theory down under (Canberra, 1996), de Gruyter, Berlin, 1999, 293-316.

[47] Ch. Pittet, L. Saloff-Coste, "On the stability of the behavior of random walks on groups", J. Geom. Anal., 10:4 (2000), 713-737.

[48] C. Pittet, L. Saloff-Coste, "On random walks on wreath products", Ann. Probab., 30:2 (2002), 948-977.

[49] R. Rammal, G. Toulouse, M. A. Virasoro, "Ultrametricity for physicists", Rev. Modern Phys., 58:3 (1986), 765-788.

[50] J. J. Rodríguez-Vega, W. A. Zúñiga-Galindo, "Taibleson operators, p-adic parabolic equations and ultrametric diffusion", Pacific J. Math., 237:2 (2008), 327-347.

[51] L. Saloff-Coste, "Opérateurs pseudo-différentiels sur certains groupes totalement discontinus", Studia Math., 83:3 (1986), 205-228.

[52] L. Saloff-Coste, "Probability on groups: random walks and invariant diffusions", Notices Amer. Math. Soc., 48:9 (2001), 968-977.

[53] R. L. Schilling, R. Song, Z. Vondraček, Bernstein functions. Theory and applications, 2nd ed., de Gruyter Stud. Math., 37, Walter de Gruyter \& Co., Berlin, 2012, xiv $+410 \mathrm{pp}$.

[54] P. M. Soardi, Potential theory on infinite networks, Lecture Notes in Math., 1590, Springer-Verlag, Berlin, 1994, viii+187 pp.

[55] M. H. Taibleson, Fourier analysis on local fields, Princeton Univ. Press, Princeton, NJ; Univ. of Tokyo Press, Tokyo, 1975, xii+294 pp.

[56] N. Th. Varopoulos, L. Saloff-Coste, T. Coulhon, Analysis and geometry on groups, Cambridge Tracts in Math., 100, Cambridge Univ. Press, Cambridge, 1992, xii+156 pp.

[57] В.С. Владимиров, “Обобщенные функции над полем p-адических чисел”, УМН, 43:5(263) (1988), 17-53; англ. пер.: V.S. Vladimirov, "Generalized functions over the field of p-adic numbers", Russian Math. Surveys, 43:5 (1988), 19-64.

[58] V.S. Vladimirov, I. V. Volovich, "P-adic Schrödinger-type equation", Lett. Math. Phys., 18:1 (1989), 43-53.

[59] V.S. Vladimirov, I. V. Volovich, E.I. Zelenov, p-adic analysis and mathematical physics, Ser. Soviet East European Math., 1, World Sci. Publ., River Edge, NJ, 1994, $\mathrm{xx}+319 \mathrm{pp}$.

[60] Z. Vondraček, "A characterization of Markov chains on infinite graphs by limiting distributions", Arch. Math. (Basel), 65:5 (1995), 449-460.

[61] W. Woess, Random walks on infinite graphs and groups, Cambridge Tracts in Math., 138, Cambridge Univ. Press, Cambridge, 2000, xii+334 pp.

[62] W. Woess, "Lamplighters, Diestel-Leader graphs, random walks, and harmonic functions", Combin. Probab. Comput., 14:3 (2005), 415-433.

[63] W. Woess, Denumerable Markov chains. Generating functions, boundary theory, random walks on trees, EMS Textbk. Math., Eur. Math. Soc., Zürich, 2009, xviii+351 pp.

[64] W. Woess, On the duality between jump processes on ultrametric spaces and random walks on trees, 2012, 24 pp., arXiv: 1211.7216. 
[65] M. Yamasaki, "Discrete potentials on an infinite network", Mem. Fac. Sci. Shimane Univ., 13 (1979), 31-44.

[66] W. A. Zúñiga-Galindo, "Parabolic equations and Markov processes over $p$-adic fields", Potential Anal., 28:2 (2008), 185-200.

\section{Александр Давидович Бендиков}

(Alexander Bendikov)

Поступила в редакцию

Institute of Mathematics, Wroclaw University,

Wroclaw, Poland

E-mail: bendikov@math.uni.wroc.pl

\section{Александр Асатурович Григорьян}

(Alexander Grigor'yan)

University of Bielefeld, Germany

E-mail: grigor@math.uni-bielefeld.de

\section{Кристоф Питтэ}

\section{(Christophe Pittet)}

LATP, Université d'Aix-Marseille, Marseille, France

E-mail: pittet@cmi.univ-mrs.fr

\section{Вольфганг Вёсс}

(Wolfgang Woess)

Institut für Mathematische Strukturtheorie, Technische Universität Graz, Graz, Austria

E-mail: woess@TUGraz.at 